University of Massachusetts Amherst

ScholarWorks@UMass Amherst

March 2020

\title{
TPR-CONTAINING PROTEINS CONTROL PROTEIN ORGANIZATION AND HOMEOSTASIS FOR THE ENDOPLASMIC RETICULUM
}

Jill Bradley-Graham

University of Massachusetts Amherst

Follow this and additional works at: https://scholarworks.umass.edu/dissertations_2

Part of the Animal Experimentation and Research Commons, Biochemistry Commons, Bioinformatics Commons, Cell Biology Commons, Developmental Biology Commons, Integrative Biology Commons, Molecular Biology Commons, and the Other Animal Sciences Commons

\section{Recommended Citation}

Bradley-Graham, Jill, "TPR-CONTAINING PROTEINS CONTROL PROTEIN ORGANIZATION AND HOMEOSTASIS FOR THE ENDOPLASMIC RETICULUM" (2020). Doctoral Dissertations. 1814. https://doi.org/10.7275/3w2g-4r67 https://scholarworks.umass.edu/dissertations_2/1814

This Open Access Dissertation is brought to you for free and open access by the Dissertations and Theses at ScholarWorks@UMass Amherst. It has been accepted for inclusion in Doctoral Dissertations by an authorized administrator of ScholarWorks@UMass Amherst. For more information, please contact scholarworks@library.umass.edu. 
TPR-CONTAINING PROTEINS CONTROL PROTEIN ORGANIZATION AND HOMEOSTASIS FOR THE ENDOPLASMIC RETICULUM

A Dissertation Presented

by

JILL BRADLEY-GRAHAM

Submitted to the Graduate School of the

University of Massachusetts Amherst in partial fulfillment

of the requirements for the degree of

DOCTOR OF PHILOSOPHY

February 2020

Molecular and Cellular Biology 
(C) Copyright by Jill Bradley-Graham 2020

All Rights Reserved 


\title{
TPR-CONTAINING PROTEINS CONTROL PROTEIN ORGANIZATION AND HOMEOSTASIS FOR THE ENDOPLASMIC RETICULUM
}

\author{
A Dissertation Presented \\ by \\ JILL BRADLEY-GRAHAM
}

Approved as to style and content by:

Daniel N. Hebert, Chair

Dominique Alfandari, Member

Peter Chien, Member

Richard Vachet, Member

Scott C. Garman

Molecular and Cellular Biology 


\section{DEDICATION}

To my Mom, Donna, for all of your love, encouragement, inspiration and firm arch support. 


\section{ACKNOWLEDGMENTS}

First, I want to thank my advisor, Dr. Daniel Hebert, for his support, scientific advice and critical thinking throughout my time here. With patience, he guided me through several difficult research hurdles, continually encouraging creativity, collaboration and practice, all of which are necessary to understand and communicate the complexity of cell biology. I would like to thank my thesis committee members, Dr. Peter Chien, Dr. Richard Vachet and Dr. Dominique Alfandari for their insightful input, advice and discussion in developing my work. I had the great pleasure of interacting with these individuals on a daily basis and learned much from these interactions. Particularly, I would like to thank Dr. Alfandari for allowing me to perform all of the embryo work in his lab, his patience in teaching me the Xenopus system and all of the help with experimental optimization and assay development.

It takes a village...to a get a Ph.D. I would like to thank my family for their love, understanding and support throughout this process. From a young age, my parents, Donna and Dan Gorman and Bruce Graham, helped us develop individual decisionmaking skills and encouraged my passion for learning. They have always supported my career decisions and helped me grow from each experience, creating an environment where I could follow any path I chose. Along with my sisters, Jan and Remi, and my grandmother, Joyce, they have been my inspiration throughout this process. In moving across the country and beginning this program, I missed countless family events, birthdays and moments of hardship. Thank you for getting me home and spending time with me during those always-too-short visits. Thank you for making me feel included 
despite being time zones apart with facetime calls and shared photo albums and thank you for the taking the time to visit me. For all of this, I am eternally grateful.

I would like to thank my partner, Bobby Loguidice, for your patience, love and support. Thank you for believing in me, getting me out of the lab, sharing food with me and being awesome all around. I would also like to thank friends and fellow students that have made this adventure an incredible experience. Thank you to my childhood friend, Gianna, and her husband Mike for your unending support, understanding, encouragement and laughter. Thank you both for including me in your major life celebrations and making 3000 miles seem like 30 at times. To Lydia Lamriben, Christina Chisholm and Michael Mingroni, you continually inspire me as fellow students and incredible humans. To Hillary Milens, Lauren Baker and Naomi Valentine for giving me a place filled with love and fun to come home to every day. To Johan and Bri Sunryd, Celia Homyak, Ilene Magpiong, Marc Cartwright, Katie Love, Kristen Sikora, Francesca Anson, Karan Hingorani, Ketan Mathavan, Rilee and Ali Zeinert and Stephanie Craig, thank you for your ever-present friendship and mentorship.

Finally, I would like to thank past and current members of the Hebert Lab. Johan Sunryd and Abla Tannous for training me, your scientific mentorship and friendship. Lydia, thank you for sharing your love of food, science and sense of humor. Thank you to Haiping for your endless patience, cloning and assay help. Working alongside all of you was a terrific experience and I have learned so much from each of you. Theresa, Doug, Yekatarina and Michela were great undergraduates showing dedication and enthusiasm from early on. I also need to thank Ben Adams, Cheyenne Hakes and Nathan Canniff for scientific discussions. 


\title{
ABSTRACT \\ TPR-CONTAINING PROTEINS CONTROL PROTEIN ORGANIZATION AND HOMEOSTASIS FOR THE ENDOPLASMIC RETICULUM
}

FEBRUARY 2020

\author{
JILL BRADLEY-GRAHAM, B.Sc., UNIVERSITY OF CALIFORNIA, SANTA CRUZ \\ Ph.D., UNIVERSITY OF MASSACHUSETTS AMHERST \\ Directed by: Professor Daniel N. Hebert
}

The endoplasmic reticulum (ER) is a complex, multifunctional organelle comprised of a continuous membrane and lumen that is organized into several functional regions. It plays various roles including protein translocation, folding, quality control, secretion, calcium signaling, and lipid biogenesis. Cellular protein homeostasis is maintained by a complicated chaperone network, and the largest functional family within this network consists of proteins containing tetratricopeptide repeats (TPRs). TPRs are well-studied structural motifs that mediate intermolecular protein-protein interactions, supporting interactions with a wide range of ligands or substrates. Nine TPR-containing proteins have been shown to localize to the ER and control protein organization and homeostasis within this multifunctional organelle. Here, we discuss the roles of SEL1L, ERdj6, FICD, TMTC1-4, TTC13 and TTC17 and how they contribute to controlling ER processes and organization. The crucial roles that TPR-containing proteins play in the ER are highlighted by diseases or defects associated with their mutation or disruption. 
TABLE OF CONTENTS

Page

ACKNOWLEDGMENTS ..$v$

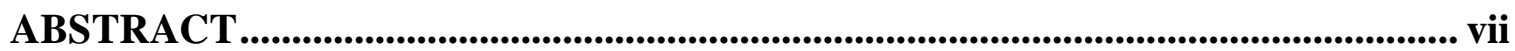

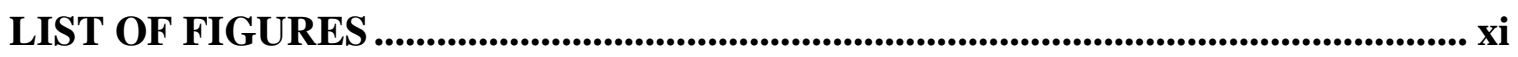

\section{CHAPTER}

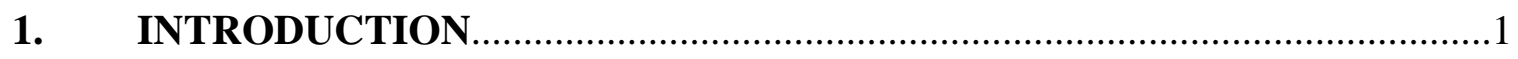

1.1 A historical perspective on endoplasmic reticulum morphology, organization

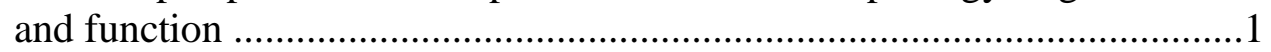

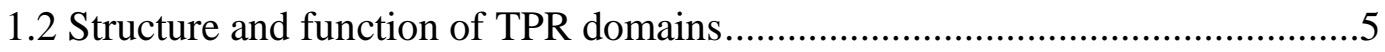

1.3 Functional roles for ER TPR-containing proteins ...........................................

1.3.1 Protein translocation across intracellular membranes..........................8

1.3.2 Molecular chaperone adaptors and regulators ..................................13

1.3.3 Protein modifications ......................................................................18

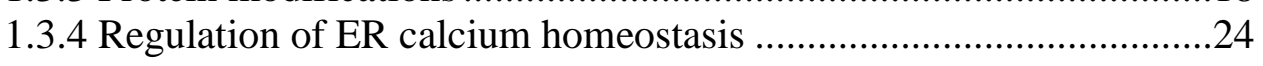

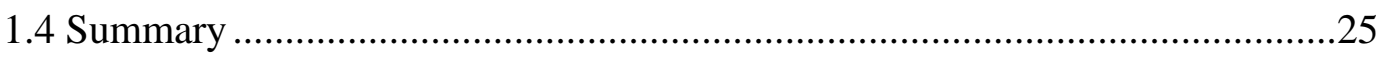

2. TMTC3 CONTRIBUTES TO O-MANNOSYLATION OF E-CADHERIN .34

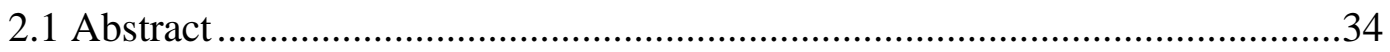

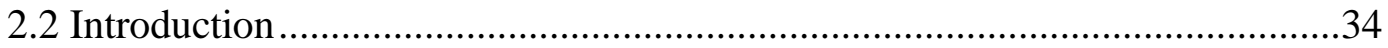

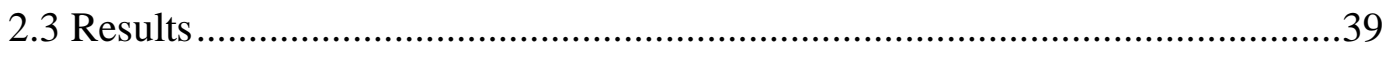

2.3.1 TMTC3 and TMTC4 are ER resident proteins ..................................39

2.3.2 TMTC3 and TMTC4 are ER membrane proteins with luminal orientated TPR motifs ..................................................................41

2.3.3 TMTC3 and TMTC4 are upregulated by folding and trafficking stress ......................................................42

2.3.4 TMTC3 rescues O-mannosylation of E-cadherin .............................44

2.3.5 TMTC3 enhances cellular adherence and binding to E-cadherin......46

2.3.6 TMTC3 disease variants exhibit reduced stability .............................49

2.3.7 TMTC3 and TMTC4 knockdown delays gastrulation in Xenopus laevis......................................................... 52

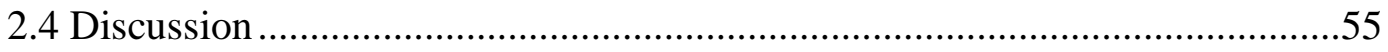

2.5 Materials and Experimental Procedures ……………....................................62 


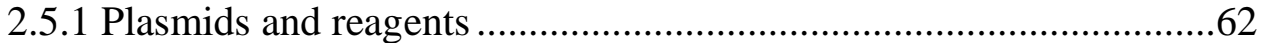

2.5.2 Cell lines/Tissue culture .............................................................63

2.5.3 In silico analysis of TMTC3 and TMTC4 ...................................63

2.5.4 Affinity purification and glycosylation assay ...............................64

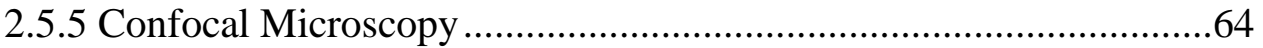

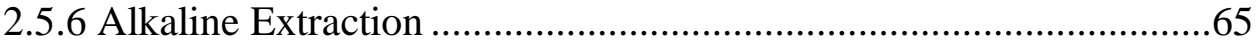

2.5.7 Trypsin protection .................................................................66

2.5.8 Immunblotting, endoglycosidase and affinity purification ...............66

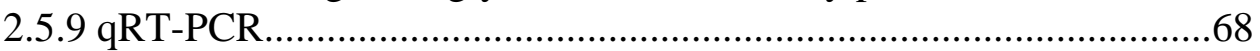

2.5.10 Cycloheximide chase analysis ..............................................69

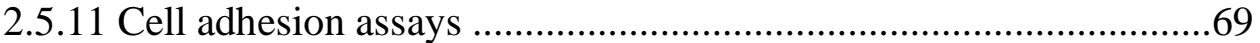

2.5.12 Xenopus embryo handling and media ........................................ 70

2.5.13 Microinjection experiments ............................................... 70

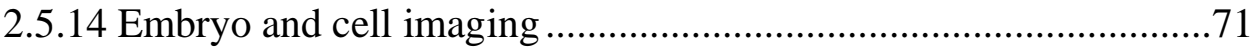

2.5.15 Protein extraction and analysis .............................................. 71

3. TTC17 IS A NOVEL ER ADAPTOR INVOLVED IN ER STRESS .............90

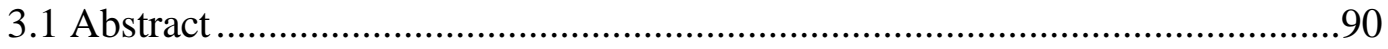

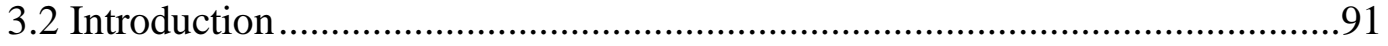

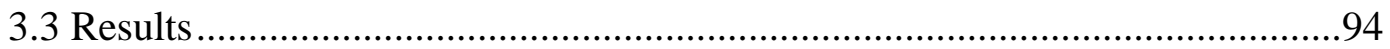

3.3.1 TTC17 is an ER resident protein ..............................................94

3.3.2 TTC17 is a soluble protein that resides within the ER lumen ..........96

3.3.3 TTC17 is upregulated by folding, calcium, trafficking and proteasomal stress .................................................................97

3.3.4 Endogenous TTC17 and ER chaperones show elevated expression

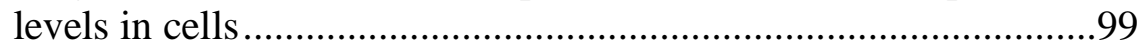

3.3.5 TTC17 interacts with a number of chaperones ............................101

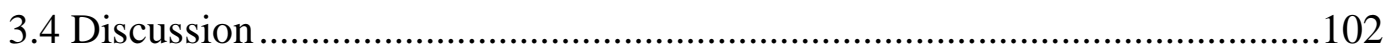

3.5 Materials and Experimental Procedures .............................................. 107

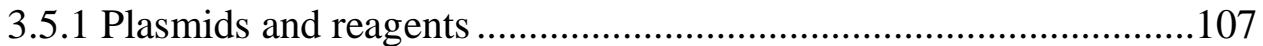

3.5.2 Cell lines/Tissue culture...........................................................107

3.5.3 In silico analysis of TTC17 ......................................................108

3.5.4 Affinity purification and glycosylation assay .............................108

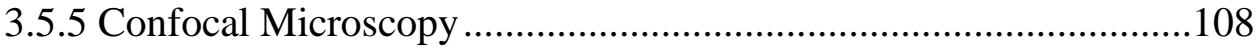

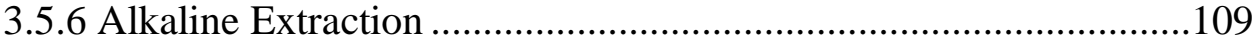

3.5.7 Trypsin protection .................................................................. 110

3.5.8 Immunblotting and affinity purification ....................................110

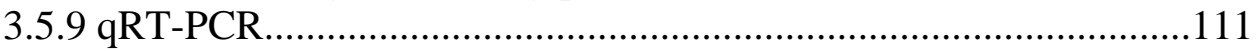

3.5.10 Shotgun LC-MS/MS and silver stain ....................................112

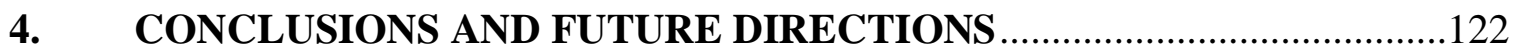


4.1 TMTC3 is a novel O-mannosyltransferase

4.2 TTC17 is a novel ER adaptor involved in ER stress ....

4.3 Summary

\section{APPENDIX: TTC13 IS A NOVEL ER RESIDENT TPR-CONTAINING}

PROTEIN.

136

BIBLIOGRAPHY 


\section{LIST OF FIGURES}

Figure

1.1. Tetratricopeptide Repeats .27

1.2. ER TPR-containing proteins .29

1.3. Sec72, SGTA and SEL1L are involved in ER protein translocation 30

1.4. ER TPR co-chaperone, ERdj6 and protein modifiers, FICD and TMTC1-4 control protein homeostasis through interactions with $\mathrm{BiP}$ and glycosylation

2.1. TMTC3 and TMTC4 are ER resident proteins .72

2.2. TMTC3 and TMTC4 are transmembrane proteins with their TPR domains facing the ER lumen .73

2.3. Basal transcript abundance and fold induction of TMTC proteins by ER stress ........74

2.4. TMTCs and POMTs share similar functional domain architecture .75

2.5. TMTC3 rescues O-mannosylation of E-cadherin in HEK293 COSMC/POMGnT1/TMTC1/2/3/4-/- cells .76

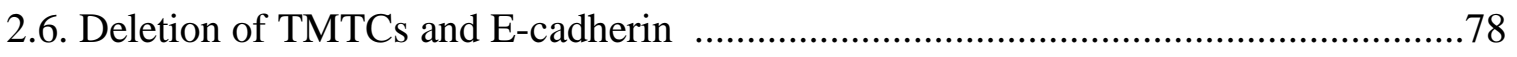

2.7. TMTC3 enhances E-cadherin mediated cellular adherence ...................................79

2.8. TMTC3 disease variant predictive outcomes and carbohydrate analysis $\quad . . . . . . . . . . . . . .81$

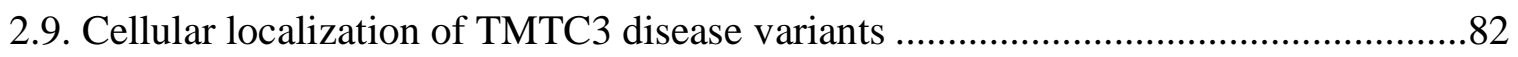

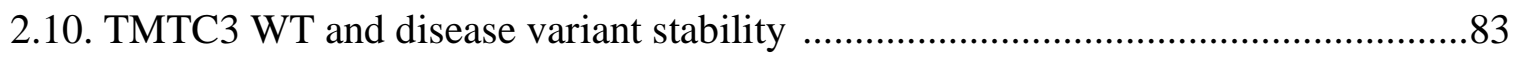

2.11. TMTC conservation in commonly studies species and RNA expression in Xenopus laevis........................................................... 84

2.12. Knockdown of Tmtc3 in developing Xenopus laevis embryos affects gastrulation 86

2.13. Putative active sites of TMTC3 .88

3.1. TTC17 is an ER resident TPR-containing protein 113

3.2. TTC17 is a soluble protein that resides within the ER lumen 115 
3.3. Basal transcript abundance and fold induction of TTC17 by ER stress

3.4. TTC17 and ER stress chaperones show elevated expression in cells

3.5. TTC17 is up-regulated by heat in reglucosylation deficient cells

3.6. ER localized S-tagged TTC17 interacts with a number of chaperones

3.7. Structural models of TTC17 TPR clusters reveal positively charged patches on the concave surface

4.1. TMTC3 and TMTC4 reside in higher molecular weight complexes and may interact with substrate via conserved Asn

A1. TTC13 is an ER resident TPR-containing protein

A2. Basal transcript abundance and fold induction of TTC13 by ER stress

A3. TTC13 is a soluble protein that resides within the ER lumen

A4. ER localized S-tagged TTC13 interacts with a number of chaperones 


\section{CHAPTER 1}

\section{INTRODUCTION}

(Sections from this chapter are adapted from (Graham et al., 2019))

\subsection{A historical perspective of endoplasmic reticulum morphology, organization and}

\section{function.}

In eukaryotic cells, cellular processes are frequently organized into specific subcompartments or organelles. The endoplasmic reticulum (ER) is comprised of a single lumen and contiguous membrane that spreads throughout the cell, commonly making it the largest organelle within a cell. The ER was first observed in 1902 by Emilio Veratti when he used Camillo Golgi's staining method and found a cellular structure distinct from the Golgi apparatus. It was not until 1953 that the ER was named and acknowledged to be an organelle. With the development of electron microscopy, Keith Porter and George Palade validated the presence of a net-like (reticulum) structure within (endo) the cytoplasm (plasmic) via high-resolution electron microscopy (EM) imaging (Palade and Porter, 1954; Veratti, 1961). Palade also developed a method to isolate ER-derived "microsomes" and in combination with microscopy-based analyses, extensive biochemical assays were performed leading to the discovery that the ER participates in a number of essential processes including protein folding, quality control, trafficking, secretion and homeostasis, calcium signaling, and lipid biogenesis and regulation (Caro, 1964; Lamriben et al., 2016; Mazzarello et al., 2003; Palade and Siekevitz, 1956; Stein and Stein, 1967; Veratti, 1961). Studies in the last few decades have shown that different 
subdomains characterized by the enrichment of specific factors exist within this singular luminal space of the ER, creating functional sub-regions (Lynes and Simmen, 2011).

Early microscopic and biochemical observations showed two distinct domains of the ER: the rough ER, characterized by the presence of ribosomes, and the smooth ER that is devoid of ribosomes. Extensive studies on the rough ER have provided numerous breakthroughs in understanding its function but progress on the smooth ER has been slower.

The rough ER membrane is easily distinguishable in EM images as it is studded with ribosomes that provides the rough appearance. Using combined EM and autoradiography, the flow of proteins from the cytoplasm, through the ER, and onwards to the Golgi was observed and provided primary evidence that the ER is the site of entry into the secretory pathway (Caro, 1964). Ribosomes are nestled onto translocons (Sec61) embedded in the ER membrane that provide the conduit for translocation into the ER lumen (Park and Rapoport, 2012). Proteins resident to the rough ER include transloconassociated factors such as proteins involved in signal sequence processing or glycosylation, as well as molecular chaperones (i.e. BiP, calnexin and calreticulin) and oxidoreductases (i.e. PDI and Erp57) that assist the folding and maturation of the newly synthesized polypeptides (Alder et al., 2005; Harada et al., 2009; Johnson and van Waes, 1999; Shibatani et al., 2005). The signal sequence peptidase and glycosyltransferase complexes, as well as chaperones and oxidoreductases, are known to participate in cotranslational events in the rough ER but they may also be observed in other regions of the ER (Chen et al., 1995; Daniels et al., 2003; Gilchrist et al., 2006; Molinari and Helenius, 1999, 2000). 
Over the last two decades, work pertaining to the smooth ER has identified and characterized several subdomains including ER exit sites (ERES) and the ER quality control compartment (ERQC-C) (Benyair et al., 2015a; Budnik and Stephens, 2009). ERES mediate the export of secretory cargo from the ER and generate the ER-Golgi intermediate (ERGIC) compartment (Jensen and Schekman, 2011). This portion of the ER is part of the transitional or intermediary ER, characterized by its overlap with the rough ER and its transition into the Golgi vesicular network. An emerging subdomain of the ER is the ERQC-C. In order to ensure proper protein processing, nascent proteins of different folding states must be segregated by distinguishing properly folded proteins at ERES for export to the Golgi from those that are terminally misfolded for targeting for ER-associated degradation (ERAD) in the ERQC-C (Benyair et al., 2015a). It has been suggested that a number of the ER quality control factors, as well as accumulated misfolded secretory cargo, are also collected into peri-centrosomal vesicles, or quality control vesicles (QVCs) (Benyair et al., 2015b). However, these membrane enclosed QVCs and the localization of ERAD and ERQC factors are not well defined.

To maintain cellular homeostasis, organelles are connected by vesicle-mediated trafficking and membrane contact sites where two heterologous membranes are closely apposed ( within $30 \mathrm{~nm}$ ) but do not fuse. The ER forms membrane contact sites with multiple membrane systems including the plasma membrane, mitochondria, Golgi, endosomes, and lipid droplets (Zhang and $\mathrm{Hu}, 2016$ ). Association of the ER with other organelles generally involves portions of the smooth ER and protein-protein interactions and/or protein-phospholipid interactions between the ER and the apposed organelle. The areas and shapes of such contacts are dynamic and may correlate with the functional 
demands of the contact, which include lipid and calcium exchange, fission and organelle movement.

The first role shown to be associated with the ER was calcium storage as $\mathrm{Ca} 2+$ was observed to be sequestered in the sarcoplasmic reticulum (the term for the ER in muscle cells) (Mazzarello et al., 2003). The mechanisms by which the ER sequesters and releases $\mathrm{Ca} 2+$ gave essential insight into the studies of muscle contraction, signaling, fertilization and neurotransmission (Hales et al., 1974; McGraw et al., 1980). Whereas some calcium binding proteins, like calreticulin, or re-uptake pumps, like SERCA, appear to be widely distributed throughout the ER, IP3 receptors and ryanodine receptor calcium channels are often found clustered within the smooth ER (Satoh et al., 1990; Takei et al., 1992).

The use of microscopic radioautography demonstrated the intracellular movement of labelled lipids, showing lipids were observed in the rough and smooth elements of the ER then in mitochondria and Golgi shortly thereafter. It was concluded that these lipid particles represented lipoproteins that formed in the ER, were processed in the Golgi and then transported into vacuoles to be dispersed into circulation (Stein and Stein, 1967). Lipid and calcium exchange and regulation between the ER and rest of the cell was later determined to be attributed mainly to the ER forming physical membrane contacts with the plasma membrane and mitochondria (Giorgi et al., 2009; Hayashi et al., 2009; Pichler et al., 2001).

The domain of the smooth ER found in close contact with the plasma membrane is termed the plasma-associated membrane (PAM) (Pichler et al., 2001). A significant role of the PAM, also known as the cortical or peripheral ER, is calcium exchange and 
lipid transfer. The mitochondria-associated membranes (MAMs) are important for energy metabolism and regulated cell-death, therefore they are pivotal for cellular function and survival. MAMs also enable highly efficient transmission of calcium from the ER to mitochondria to stimulate oxidative metabolism (Boehning et al., 2003; Rizzuto et al., 1998; Simmen et al., 2010). Additionally, the smooth ER is now known to provide the point of origin for entire organelles such as the peroxisome, Russell bodies, and lipid droplets (Kalantari et al., 2010; Kopito and Sitia, 2000; Mast et al., 2010).

A multitude of cellular functions rely on adaptor proteins and multi-protein complexes to mediate compartmentalization and organellar contact sites. A number of ER resident factors utilize protein-protein interaction motifs to nucleate multi-protein complexes directing, at least in part, the formation of ER subdomains (Zhang and $\mathrm{Hu}$, 2016). A better understanding of the employment of adaptor proteins and protein-protein interaction motifs could lead to a more complete understanding of ER functional organization. This thesis aims to describe how proteins containing TPR motifs contribute to the roles and functional organization of the ER.

\subsection{Structure and function of TPR domains}

Adapter or scaffold proteins commonly utilize repeat motifs to nucleate protein complexes since repeats allow a variety of binding surfaces with minimal sequence variation. They are comprised of tandem repetitions of a short structural motif and have been used as molecular recognition tools in a wide variety of applications (Sawyer et al., 2013). Examples of repeat proteins are Leucine Rich Repeats (LRR), ankyrin repeats (Ank), the WD40 repeat, the armadillo repeat (ARM), and the TPR. Each of these motifs has its own signature, a conserved set of amino acids that specify the repeat structure 
(Good et al., 2011; Sawyer et al., 2013). The TPR is of particular interest because they are found in numerous proteins, serving as interaction modules and multi-protein complex mediators, and recently TPR-containing proteins were found to comprise approximately one third of the mammalian protein chaperone network in the cell (Brehme et al., 2014; Zeytuni and Zarivach, 2012).

Since their identification, TPR-containing proteins have been found in all kingdoms of life and to regulate a number of diverse biological processes including protein folding and import, organelle targeting and vesicle fusion. A single TPR motif consists of 34 amino acids in a degenerate consensus sequence defined by a jig saw pattern of small and large hydrophobic amino acids. Though this general consensus sequence or pattern describes TPR motifs, no residue is invariant; however, residue type is highly conserved at three positions $(8,20$ and 27$)$ relative to the motif's N-terminal residue (D’Andrea and Regan, 2003; Magliery and Regan, 2005).

The canonical unit of the TPR adopts a basic helix turn helix fold, and adjacent TPR units form a series of repeating antiparallel $\alpha$-helices (Figure 1.1A). This yields an overall super-helical structure affected by the residue found between adjacent motifs and the number of repeats next to one another. A positive correlation was determined between protein thermostability and the number of TPR motifs using a non-natural recombinant TPR-containing protein with 20 sequential TPRs (Kajander et al., 2007). Additionally, increasing numbers of TPR motifs confer kinetic folding cooperativity and were shown to involve the thermodynamic interplay between the stability of individual repeats as well as the interaction between repeats (Javadi and Main, 2009). 
TPR-containing proteins use different binding pockets to bind diverse ligands that usually do not share secondary structure or sequence similarities. Although a defined rule set is lacking, binding is usually highly specific. The crystal structures of many TPRdomain containing proteins show two anti-parallel $\alpha$-helices packed in tandem arrays to form a structure with an amphipathic groove which can bind a target peptide (Grove et al., 2008; Zeytuni and Zarivach, 2012). This is not, however, the only mode of target recognition by TPR domains, with short amino acid insertions and alternative TPR motif conformations also shown to contribute to protein interactions, highlighting diversity in TPR domains and the versatility of this structure in mediating biological events (Allan and Ratajczak, 2011). In nature, TPR motifs can be found in tandem arrays of 2-16 sequential motifs (D’Andrea and Regan, 2003; Jiménez et al., 2012). Clusters of three TPR tandem motifs are the most common, and they form a distinct curve, binding the ligand on the concave side (Figure 1.1B) (Scheufler et al., 2000). The binding of ligands for clusters of three TPRs seems to be more specific, whereas when higher numbers of repeats are found adjacent to one another, the binding is more promiscuous (D'Andrea and Regan, 2003; Jínek et al., 2004).

To obtain such diverse binding, the TPRs utilize their distinct fold to serve as an interaction platform. This platform can exhibit different surface residues in each binding surface, yielding specificity by a combination of factors (Zeytuni and Zarivach, 2012). Residue type affects the electrostatic nature of the binding surface by contributing positive and negative charges. Additionally, residues with different hydrophobicity and size can support hydrophobic interactions between TPR domains and ligands. The secondary structure of TPR-bound ligands varies between extended coil to $\alpha$-helix 
conformation, or a combination of the two. Elongated conformation maximizes the ligand surface presented to the TPR domain, as well as optimizes H-bond formation and thereby facilitates the specific recognition of the short amino acid stretches. Given the complicated organization of the ER, which in part is directed by the formation of multiprotein complexes, and TPR-containing proteins possessing intracellular signaling scaffold, spatial organization, and assembly-line properties, TPRs may contribute to the ER's various functions. Nine proteins containing TPRs have thus far been shown to localize to the ER and contribute to its diverse cellular functions (Figure 1.2) (Kaneko and Nomura, 2003; Li et al., 2018; Preissler et al., 2015; Racapé et al., 2011; Sunryd et al., 2014; Yan et al., 2002). The roles and functions of these nine proteins will be discussed below.

\subsection{Functional roles for ER TPR-containing proteins}

\subsubsection{Protein translocation across the intracellular membranes}

Protein translocation is a process by which a polypeptide chain moves across a membrane (Blobel, 1980; Schnell and Hebert, 2003). The protein is frequently directed to its target membrane by an $\mathrm{N}$-terminal signal sequence and protein translocation can occur co-translationally or post-translationally. TPR-containing proteins have been shown to be essential for a number of cellular translocation processes as cells or organisms lacking these proteins exhibit growth and survival defects (Harkness et al., Nieuwkoop et al., 1994; Sun et al., 2014). In order to better understand the function of TPR-containing proteins involved in translocation across the ER membrane, we first describe one of the well-studied TPR-containing protein import systems involving the translocation of 
proteins across the outer mitochondrial membrane to provide framework for translocational roles.

The majority of mitochondrial proteins are synthesized by cytosolic ribosomes and then imported into the organelle post-translationally. TOM (the translocase of the outer mitochondrial membrane) is a multi-protein complex that contains surface receptors for pre-protein recognition and a translocon that serves as the membrane conduit to cross the outer mitochondrial membrane. The translocase complex is comprised of at least seven proteins, including TOM70 and TOM20, both of which contain TPR motifs. Mitochondrial targeting requires the cytosolic molecular chaperones Hsp70 and Hsp90, and their chaperone activity and substrate binding is regulated by a number of cochaperones, of which some contain TPR motifs (discussed below in the chaperone networks section). TOM70, the mitochondrial import receptor, is also a member of the TPR co-chaperone family as it contains a conserved TPR-clamp domain comprised of three TPR motifs for interaction with Hsp70 and Hsp90 (Young et al., 2003). Cellular studies on this interaction between human TOM70 and chaperones has shown it to be important for preprotein import preparation and the initiation of the import process (Fan et al., 2006). TOM70 also contains an additional two clusters of TPRs (each containing three TPR motifs), which may be involved in preprotein recognition and translocation (Wu and Sha, 2006). TOM20 contains a single TPR motif, which is unique in TPR functional analysis. The structures of both rat and yeast TOM20 reveal that the single TPR motif along with a third helix form a hydrophobic groove, which is the binding site for $\mathrm{N}$-terminal presequence peptides aiding in transport cargo recognition (Muto et al., 2001). These findings show the important roles that TPR-containing proteins play in 
protein import and how the different TPR functional domains exhibit various binding specificities.

In the secretory pathway, TPR-containing proteins have been shown to be crucial for protein translocation both into and out of the ER (Figure 1.3). ER post-translational translocation in yeast requires the Sec61 translocon channel and a complex of 4 additional proteins: Sec63, Sec62, Sec71 and Sec72 (Itskanov and Park, 2019; Wu et al., 2018). Using a combined structural and biochemical approach, the role of the $C$. thermophilum Sec71/72 subcomplex was determined (Tripathi et al., 2017). The crystal structure reveals that Sec72 contains a TPR domain that has multiple chaperone interaction sites and is anchored to the ER membrane by Sec71 and Sec63. This TPR domain interacts with the C-terminus of Ssa1, cytosolic Hsp70, which binds to the concave inner surface of the TPR domain. Surprisingly it also interacts with Ssb1, a cytoplasmic Hsp70 that binds ribosome associated nascent polypeptide chains even though it lacks the C-terminal TPR-binding residues of Ssa1. Ssb1 instead interacts with the TPR domain through its ATPase domain along the backside (convex) of the TPRs allowing for translocation substrates to be recruited to the Sec71/72 complex both co- and post-translationally (Tripathi et al., 2017). Recent cryo-EM data reveals that Sec63 positions Sec71/72 for the capture of polypeptides associated with cytosolic Hsp70, an interaction mediated by the TPR domain of Sec72 (Figure 1.3A) (Itskanov and Park, 2019; Wu et al., 2018). Notably, Sec71/Sec72 genes are restricted to fungi and this posttranslation translocation process has been worked out in yeast. A similar process is expected to occur in metazoans to a lesser extent, however proteins other than Sec71/72 may be involved in recruiting Hsp70/90 chaperones. 
Another TPR-containing protein involved in a specialized post-translational membrane integration process is small glutamine-rich TPR-containing protein alpha (SGTA). SGTA acts as a co-chaperone within a complex of proteins, including BAG6 and TRC40 (Get3 in yeast), that facilitates the targeting of tail-anchored membrane proteins to a Golgi to ER traffic (GET) membrane integration complex in the ER membrane, as well as the sorting of membrane and secretory proteins that mislocalize to the cytosol for degradation (Martínez-Lumbreras et al., 2018). In combination with the BAG6 complex, SGTA helps to perform molecular triage to regulate the fate of tailanchored (TA) membrane proteins and mislocalized secretory cargo by binding hydrophobic substrates and transferring selected protein clients to the BAG6 complex for sorting (Figure 1.3B) (Shao et al., 2017). SGTA is comprised of an N-terminal dimerization domain, a central domain consisting of three TPR motifs and a C-terminal substrate binding motif (Chartron et al., 2011; Darby et al., 2014; Dutta and Tan, 2008; Martínez-Lumbreras et al., 2018; Simon et al., 2013). The TPR domain interacts directly with Hsp70 and Hsp90 chaperones, as well as the proteasomal subunit, Rpn13 (Leznicki et al., 2015; Minami et al., 2010; Thapaliya et al., 2016). The TPR domain gives SGTA the co-chaperone-like ability to participate in both productive "on-pathway" folding as well as protein degradation.

As proteins enter the secretory pathway, they are assessed by a number of ER resident quality control factors to determine if they are properly folded or require additional folding attempts (Ellgaard and Helenius, 2003; Tannous et al., 2015). If a protein is deemed terminally misfolded, it is targeted for ERAD, which requires retrotranslocation from the ER to the cytoplasm for polyubiquitination and subsequent 
degradation by the proteasome. SEL1L co-localizes with HRD1, one of the ER E3 ligases involved in ERAD (Gardner et al., 2000). It also associates with additional ERAD factors including OS-9 and GRP94 (the ER Hsp90) that have been shown to deliver mutant $\alpha 1$ antitrypsin to the HRD1-SEL1L ubiquitin complex for degradation, confirming its role in ERAD (Figure 1.3C) (Christianson et al., 2008).

The recently solved structure of SEL1L provides insight into how it contributes to the ERAD process (Jeong et al., 2016). SEL1L is comprised of a fibronectin type II domain at the N-terminus followed by eleven SEL1L-like repeat (SLR) motifs and a Cterminal transmembrane domain (Figure 1.2). The SLR motif shares a similar antiparallel $\alpha$-helical structure with the TPR motif, but the consensus sequence length is extended (36-44 amino acids compared to 34) (Mittl and Schneider-Brachert, 2007). Studies on SEL1L from mus musculus show that the SLR motifs make up the major portion of the luminal domain of SEL1L and are isolated into three clusters consisting of 4 SLRs (SLR-N(terminal) includes repeats \#1-4), 5 SLRs (SLR-M(12dle) repeats \#5-9) and 2 SLRs (SLR-C(terminal) repeats \#10 and 11). SLR \#9 of the SLR-M domain was shown to be required for the homo-oligomerization of SEL1L (Jeong et al., 2016). The SLR-C domain is responsible for mediating the interaction with a luminal portion of HRD1. Additionally, in yeast, SLR-C has been shown to mediate interactions with Yos9 (OS-9 homologue) as truncation mutants in Hrd3p (the SEL1L homologue) lacking this region were no longer able to bind Yos9 (Figure 1.3C) (Gauss et al., 2006). The Nterminal cluster contains 4 motifs and sequence analysis suggests that it may bind a specific partner or act as a classic co-chaperone (D’Andrea and Regan, 2003). The SLR domains in SEL1L have been shown to support homo-oligomerization or complex 
formation with other ERAD components; however, the role of the N-terminal SLR domain is still unknown.

Together, TPR and SLR-domain containing proteins aid in nucleating interactions at the ER membrane that support the translocation of proteins into and out of the ER lumen. The TPR domains in Sec72 and SGTA both recruit Hsp70/Hsp90 chaperones to complexes that mediate targeting of proteins to the ER for quality control, therefore promoting efficient organization of specific substrates and productive folding. The SLR domains of SEL1L contribute to multi-protein complex formation and recruitment of necessary ERAD factors so that terminally misfolded proteins do not accumulate, maintaining cellular protein homeostasis in a complex environment.

\subsubsection{Molecular chaperone adapters and regulators}

A main role for TPR-containing proteins in the cell is nucleating chaperone macromolecular complexes and regulating their activity (Brehme et al., 2014; Caplan, 2003). Hsp70 and Hsp90 are major molecular chaperones in the eukaryotic cytosol, playing essential roles in protein quality control by preventing aggregation, catalyzing productive folding of newly synthesized proteins and promoting degradation of misfolded polypeptides (Hartl et al., 2011; Hideaki and Yohtalou, 1991). Co-chaperones of Hsp70 and Hsp90 are characterized as non-client binding partners that participate in their function and regulation (Caplan, 2003). Hsp70 and Hsp90 cooperate with co-chaperones during the process of protein folding and require the help of co-chaperones containing TPR motifs for many of their functions. Isoforms of Hsp70 and Hsp90 exist within the different organelles, often performing similar functions. The ER contains both Hsp70 and Hsp90 paralogs called BiP/GRP78 and GRP94, respectively. Both BiP and GRP94 have 
been shown to localize to both the rough and smooth ER as they are some of the most abundant proteins within the organelle, participating in quality control, productive folding and stress response. As such, they are often recruited to sub-regions of the ER by cochaperones dictating their role in maintaining protein homeostasis and one of the cochaperones contains TPRs (discussed below).

To better understand how TPR-containing proteins act as co-chaperones, we first describe cytosolic TPR-containing proteins because their roles as co-chaperones have been extensively characterized. One of the most well-studied TPR-containing proteins, Hsp70/Hsp90 Organizing Protein (HOP), mediates the spatial proximity of Hsp70 and Hsp90 by associating with both chaperones and facilitating the passage of cargo from one major chaperone to the other for efficient protein folding. HOP is a monomeric protein composed of nine TPR motifs, clustered into three distinct TPR domains (Odunuga et al., 2004; Yi et al., 2010). Both chaperone sequences end with the motif EEVD and this tetrapeptide is recognized by the HOP TPR1 and TPR2A domains for Hsp70 and Hsp90, respectively. Crystal structures of the TPR-peptide complexes show peptides spanning the groove in the concave surface of the TPR domains and binding is mediated by electrostatic interactions with the EEVD motif with the C-terminal aspartate acting as a two-carboxylate clamp (Figure 1.1B) (Scheufler et al., 2000). The hydrophobic contacts between the peptide residues upstream of EEVD and the TPR domain backbone are critical for specificity.

These structural studies gave essential insight into how TPR co-chaperones are able to recruit cytosolic Hsp70 and Hsp90 and subsequent studies have identified numerous TPR co-chaperones. Unlike HOP, which uses two TPR domains to bring 
Hsp70 and Hsp90 together, other TPR co-chaperones with a TPR domain for Hsp chaperone binding often contain a different domain (i.e. E3 ligase) that targets the Hsp chaperone and its bound substrate for a specific process. Additionally, studies identified selective binding between chaperone-TPR pairs (Assimon et al., 2015). In vitro studies found that the TPR co-chaperones HOP, CHIP and DNAJC7 bound both Hsp70 and Hsp90 with similar affinities but co-chaperones FKBP51 and 52 preferably bound Hsp90. The intrinsic affinity and post-translational modifications tune the interactions between the Hsp70 and Hsp90 proteins and their TPR co-chaperones. Phosphorylation of the Hsp70 or Hsp90 C-termini significantly decreased their binding affinity to CHIP. Many of the Hsp70 and Hsp90 co-chaperones contain functional domains outside of the TPR region that direct the localization or function of their requisite chaperone-TPR pairs, which could explain selective binding between these chaperone families and the distinct roles they play within the cell.

Although it is likely that more than 100 co-chaperones exist in mammals, it appears that of the ones identified and analyzed, the proteins fall into two classes, those that contain a J-domain and those that contain TPR motifs (Caplan, 2003). Erdj6 (also known as P58IPK) is an ER resident protein that contains both features (Figure 1.2). The crystal structure of human Erdj6 revealed three N-terminal TPR domains, containing three TPRs each (similar to that found for HOP), and a C-terminal J-domain (Svärd et al., 2011). Interestingly, studies in mice show Erdj6 can be found in complex with the ER Hsp70 paralog, BiP (Figure 1.4A) (Rutkowski et al., 2007). Evidence of its role in secretory protein maturation and quality control was provided when it was found to coimmunoprecipitate with a newly synthesized secretory protein in cells. A chaperone-like 
role for Erdj6 was further supported by the fact that protein maturation was stimulated upon its overexpression and knockout cells showed decreased protein synthesis under both normal and stressed conditions; and its expression was induced by ER stress (Rutkowski et al., 2007; Yan et al., 2002).

Even though many of the cytosolic TPR co-chaperones interact with the Cterminal EEVD motif of Hsp70 or Hsp90, Erdj6 most likely binds to BiP via its Cterminal J-domain. Studies of classic Hsp chaperone systems in bacteria (E. coli) have shown that J-domain containing proteins have the ability to affect chaperone substrate binding by regulating nucleotide binding (Liberek et al., 1991). Mammalian Erdj6 contains the critical Hsp70 binding tripeptide motif (HPD) in its C-terminal J-domain (Svärd et al., 2011). The N-terminal TPR cluster contains a conserved hydrophobic patch that may be involved in binding misfolded polypeptides. This conserved hydrophobic patch is $100 \AA$ away from the BiP binding motif in the J-domain and structure-based mutagenesis for the conserved hydrophobic residues significantly reduced the molecular chaperone activity of Erdj6 (Figure 1.4A) (Tao et al., 2010). A flexible linker between the TPR subunits and the J-domain could allow movement suggesting that substrates bound to the hydrophobic surface/cleft in the TPR cluster could be brought to BiP bound at the C-terminal end (Svärd et al., 2011). Additionally, mouse Erdj6 constructs lacking the Jdomain expressed in cells still bind misfolded substrate, suggesting that the $\mathrm{N}$-terminal TPR motifs can bind polypeptides (Petrova et al., 2008).

As there has been estimated to be over 114 TPR-containing proteins in the chaperome (Brehme et al., 2014), it is likely that there are TPR-containing proteins in addition to Erdj6 and SEL1L that contribute to the organization and regulation of the 
chaperone network in the ER. Among the 114 proteins in the TPR-containing chaperone family, two proteins, TTC13 and TTC17, were simultaneously identified by a bioinformatic screen we performed for novel secretory adaptor proteins as putative TPRcontaining proteins that are potentially targeted to the ER by an N-terminal signal sequence (Figure 1.2). TTC13 remains uncharacterized and a study conducted in 2014 suggested that TTC17 is involved with actin organization and ciliogenesis in zebrafish and humans based on an interaction between TTC17 and C2orf62, which was determined using only a small GST-tagged purified region of TTC17 (aa 945-1041), excluding TTC17's N-terminal ER targeting sequence (Bontems et al., 2014). However, our data shows that both TTC17 and TTC13 reside within the ER (Figure 3.1 and Appendix A1). The function for either protein is not yet understood. Interestingly, the organization of the predicted TPR motifs in both proteins indicates that they may act as co-chaperones. TTC13 contains seven predicted TPR motifs; one near the $\mathrm{N}$-terminus of the protein followed by six consecutive motifs (Figure 1.2) (Karpenahalli et al., 2007). The six consecutive motifs are similar to the closely adjacent clusters of three motifs seen in Erdj6. TTC17 contains eight predicted TPRs according to TPRPred, one in the Nterminal portion of TTC17, a cluster of three TPRs in the middle and a cluster of four TPRs at the C-terminus. Although TTC17 is much larger than HOP, the two clusters could coordinate chaperone spatial organization and activities in a similar manner to HOP. Given that many of the ER localized soluble folding factors possess the C-terminal ER retention motif, KDEL, it will be of special interest to determine if this sequence, in analogy to the C-terminal EEVD motif of Hsp70 and 90 mediating HOP binding, directs 
binding to an ER adapter, like TTC13 or TTC17, that might help support ER organization and retention.

\subsubsection{Protein modifications}

Protein modifications involve covalent additions to proteins that expands their function and regulation (Bürkle, 2001; Truttmann and Ploegh, 2017). Protein modifications play fundamental roles in regulating protein folding, targeting, interaction with ligands or protein partners, functional states and stability. TPR-containing proteins contribute to protein regulation and function through post-translational modification in the ER by modifying chaperones with small molecules or by glycosylation (Figure 1.4) (Schjoldager and Clausen, 2012; Truttmann and Ploegh, 2017).

$\mathrm{BiP}$ is the ER member of the Hsp70 family and it relies on a number of partners, including J-domain co-chaperones (Hsp40 family members), nucleotide exchange factors, and signal transducers for its various activities (Dudek et al., 2009; Pobre et al., 2018). The activity of BiP is also modulated by AMPylation by the TPR-containing ER protein FICD (filamentation-induced by cyclic AMP domain containing protein) (Figure 1.4A) (Preissler et al., 2015, 2016a). AMPylation, also known as adenylation, is the process whereby adenosine monophosphate (AMP) from an ATP molecule is transferred to either a Tyr, Ser or Thr residue on the target protein. AMPylation of eukaryotic proteins is intimately related to the presence of FIC domains (Faber et al., 1998). FIC proteins are identifiable by their $\operatorname{HXFX}(\mathrm{D} / \mathrm{E})(\mathrm{G} / \mathrm{A}) \mathrm{N}(\mathrm{G} / \mathrm{K}) \mathrm{RXXR}$ motif, which is the domain responsible for AMPylating substrate (Garcia-Pino et al., 2014). FICD, also known as Huntingtin interacting protein E (HYPE), is the only FIC-domain containing protein in the human genome and it also contains TPR motifs (Worby et al., 2009). BiP is a 
substrate of FICD (Sanyal et al., 2015). FICD expression was upregulated under stress conditions and its knockdown prevented induction of the unfolded protein response (UPR). BiP is AMPylated at residue Thr518 in the substrate binding domain leading to its inactivation (Preissler et al., 2015, 2016b). This modification is reversible as shown by the regulatory residue, Glu234, on FICD that switches the AMPylation/de-AMPylation reactivity (Preissler et al., 2017). When the cell is under normal homeostatic conditions, FICD AMPylates BiP to create an inactive BiP pool. As the UPR is activated, FICD deAMPylates BiP so that it may engage unfolded substrate providing a rapid method to deploy active chaperones.

FICD is an ER resident type II membrane protein comprised of an N-terminal transmembrane (C) domain followed by two TPR motifs, a linker region and a C-terminal FIC domain (Figure 1.2). The FIC domain exhibits well-conserved structure with Pfam canonical four $\alpha$-helical FIC domain structure (Garcia-Pino et al., 2014). Dimerization of FICD can occur, leaving both of the FIC domains open for substrate binding and the TPR motifs available for protein-protein interactions; however, the interacting partners of the TPR domain have yet to be determined (Bunney et al., 2014). FICD's reversible ampylation of $\mathrm{BiP}$ contributes to tunable chaperone activation and productive folding.

Glycosylation, the covalent addition of carbohydrate molecules to a functional group on polypeptides, is one of the most abundant and diverse protein modifications observed in the cell (Schjoldager and Clausen, 2012). Glycosylation can promote favorable folding energetics and lower aggregation propensity to help support proper protein folding (Hebert et al., 2014; O’Connor and Imperiali, 1996; Price et al., 2012; Solá and Griebenow, 2009). Two general types of glycosylation occur, N- and O-linked 
glycosylation, whereby saccharides are attached either to the amide group of an Asn residue (N-linked) or to the hydroxy of Ser or Thr residues (O-linked) (Ecker et al., 2003). Approximately one third of the mammalian proteome is targeted to the secretory pathway, and the majority of these proteins are glycosylated (Apweiler et al., 1999). While N-linked glycosylation is the most common modification in the ER, Oglycosylation also occurs here. Recently, a family of membrane proteins containing long stretches of TPR domains was found to be involved in O-mannosylation in the ER.

O-linked glycosylation takes place in several parts of the cell. The main pathway is located in the Golgi, however O-mannosylation begins in the ER. It is catalyzed by ER resident protein O-mannosyl transferases most commonly involving POMT1 and POMT2 (Figure 1.4B) (Ecker et al., 2003). These carbohydrate chain modifications can be used to monitor glycoprotein movement through the secretory pathway, acting as a maturation and quality control tag to indicate the status of the folding polypeptide in a manner similar to that previously observed for $\mathrm{N}$-linked glycans (Hebert et al., 2014; $\mathrm{Xu}$ and Ng, 2015).

A new class of TPR-containing proteins that localize to the ER has recently been revealed: transmembrane TPR-containing proteins (TMTC) 1-4 (Sunryd et al., 2014). TMTC1-4 contain homologous structural elements as their $\mathrm{N}$-terminal halves are comprised of a number of hydrophobic domains and the C-terminal halves are composed mainly of TPR motifs (Figure 1.2). Although the structures for TMTC1-4 have yet to be determined, the predictive architecture could be compared to that of the POMT proteins and other O-linked transferases (Figure 1.4B). Both TMTC1-4 and POMT1/2 contain numerous hydrophobic segments anchoring them to the ER membrane. According to 
studies performed on yeast PMT genes, this positions the active site of the transferase close to the membrane to potentially enable the efficient transfer of the mannose from the dolichol-mannose precursor embedded in the membrane to the substrate (Bai et al., 2019). In order to attach, modify, or recognize glycoproteins, folding factors and machinery often possess multiple substrate recognition motifs or domains mediating specificity. In the POMT proteins, three MIR domains, named for the three proteins in which they occur (Mannosyltransferase, Inositol 1,4,5-triphosphate receptor (IP3R) and Ryanodine receptor), are present on a loop that faces the ER lumen and putatively bind substrates or chaperones (Fujimori et al., 2017). In contrast, the TMTC proteins possess 8-10 consecutive TPR motifs in place of the MIR domains that potentially interact with a broad range of target substrates as observed for other O-linked transferases such as $\mathrm{O}$ linked N-acetylglucosamine (GlcNAc) transferase (OGT). Information on the function of the TPRs of OGT may provide the framework for understanding the role of the TPRs in the TMTCs.

OGT resides in the cytoplasm and catalyzes the transfer of GlcNAc from UDPGlcNAc to Ser and Thr of cytoplasmic, nuclear and mitochondrial proteins including numerous transcription factors, tumor suppressors, kinases, phosphatases and histonemodifying proteins (Shafi et al., 2000; Yang et al., 2002). OGT contains an N-terminal domain comprised of 12.5 TPRs that mediates the recognition of a broad range of target proteins, as well as a C-terminal glycosyltransferase domain (Iyer and Hart, 2003; Lubas et al., 1997). The crystal structure of the homodimeric TPR domain of OGT, containing 11.5 of the consecutive TPR motifs, displays an elongated superhelical structure (Figure 1.1C, left panel). The concave surface of the superhelix is lined by conserved Asn, in a 
manner reminiscent of the peptide binding site of importin- $\alpha$, suggesting that this helical structure might help select and recruit substrates for modification (Figure 1.1C, right panel). Therefore, it would follow that the TPR domains of TMTC1-4 might also be used for substrate selection and positioning. TMTC1-4 have been implicated in the Omannosylation of the cadherin family of proteins as their knockout shows a significant reduction in the number of modified O-mannosylated sites within this family of proteins (Larsen et al., 2017a). However, active sites and direct transfer of O-mannose from the TMTCs to cadherins as well as interactions between the TMTCs and these proteins has yet to be determined. With further characterization, it will be of interest to determine if the TPR domains of TMTC1-4 can specifically bind the repeat domains of cadherins.

Though similar in composition, TMTC1-4 may play varying roles within the ER as they are associated with unique disease phenotypes. Interestingly, TMTC3 is implicated in two diseases that appear to involve O-linked glycosylation. Biallelic mutations in human TMTC3 are associated with recessive forms of cobblestone lissencephaly $(\mathrm{COB})$, a severe brain malformation due to the over-migration of neurons and glial cells (Jerber et al., 2016). The cause of the over-migration defect is impaired interaction between glial limitans and the extracellular matrix (ECM), which is dependent upon glycosylated cell surface proteins making physical linkages between the cytoskeleton of glial cells and the ECM. Coincidentally, heterozygous variants of TMTC3 were also identified in periventricular nodular heterotopia (PVNH), another disease resulting in defects in neuronal migration shown to cause brain malformations (Farhan et al., 2017). Expression analysis in patient-derived cells confirmed reduced transcript and protein levels of TMTC3. Neuron-specific knockdown of Tmtc3 in flies resulted in 
increased susceptibility to induced seizures. This phenotype was rescued by neuronspecific expression of human TMTC3, suggesting a role for TMTC3 in synaptic cells and seizure biology.

A mutation in TMTC2 (Val381Ile) is linked to nonsyndromic sensorineural hearing loss (SNHL) (Runge et al., 2016). Exome sequencing, lineage and association analyses identified a fully penetrant sequence variant in the $T M T C 2$ gene region that is associated with SNHL in a nine-family member cohort. This same variant was then found in a group of 363 unrelated individuals and associated with SNHL. TMTC4 was also linked to hearing loss in mice as tmtc4 inactivation in the cochlea caused acquired postnatal hearing loss (Li et al., 2018). After demonstrating a direct link between the more common noise-induced hearing loss (NIHL) and the UPR, mice with homozygous inactivation of both Tmtc4 and Chop had less hearing loss than knockout of Tmtc4 alone using inverse genetic complementation.

Post-translational modifications frequently affect protein function via changes in the protein structure and dynamics (Li et al., 2010). Post-translational modifications participate in a number of roles such as gene expression regulation, mediation of proteinprotein interactions and, as demonstrated above by ER TPR-containing proteins, activation or deactivation of enzymatic activities or protein stability or destruction. As modulators of cell signaling and regulation, they perform a crucial role in maintaining cellular homeostasis. Diseases linked to post-translational modifications often involve mutations of post-translational target sites, however, as exhibited above, diseases can result from mutations in the protein modifiers themselves (Jerber et al., 2016; Lazarus et al., 2011; Li et al., 2018; Runge et al., 2016). Specifically, mutations in both the active 
sites as well as TPR region result in disease (Jerber et al., 2016; Lazarus et al., 2011; Pravata et al., 2019).

\subsubsection{Regulation of ER calcium homeostasis}

Intracellular calcium is a universal second messenger in mammalian cells and chronic changes in $\mathrm{Ca} 2+$ signaling contribute to the pathogenesis of many diseases (Glaser et al., 2018). Ca2+ concentrations vary within the cell (10-100 nM) generally being lowest in the cytoplasm. One of the major functions of the smooth ER is calcium storage and it contains a host of factors dedicated to sensing and regulating intracellular calcium concentrations communicating with the cytoplasm, mitochondria and extracellular environment through the plasma membrane to maintain homeostasis (Lunz et al., 2019). The ER is also able to release stored $\mathrm{Ca} 2+$ through the ER resident IP3 and ryanodine receptors and upon depletion, the ER Ca2+ re-uptake channel, SERCA, can refill the depleted stores. TPR-containing proteins have been shown to alter cytoplasmic calcium levels by interacting with and modulating the activity of SERCA (Sunryd et al., 2014).

TMTC1, TMTC2 and TMTC4 were identified and characterized as ER-localized, transmembrane proteins involved in calcium homeostasis (Li et al., 2018; Sunryd et al., 2014). They interact with the calcium re-uptake channel, SERCA2B, while TMTC2 and TMTC4 also bound the carbohydrate binding chaperone, calnexin. TMTC1, 2 and 4 regulate the ability of SERCA to sequester $\mathrm{Ca}_{2}+$ back into the ER after stimulated $\mathrm{Ca} 2+$ release. Live cell $\mathrm{Ca}_{2}+$ measurements revealed that overexpression of either TMTC1 or 2 caused a reduction of $\mathrm{Ca} 2+$ present in the cytoplasm following stimulation suggesting increased retention or re-uptake, whereas knockdown of either or deletion of TMTC4 increased $\mathrm{Ca} 2+$ in the cytoplasm indicating less re-uptake by the ER. Additionally, 
$T M T C 2$ is differentially expressed in patients receiving serotonin re-uptake inhibitor (SSRIs) treatment for depression over the course of eight weeks (Madsen et al., 2018). TMTC2 was among other genes involved in calcium homeostasis that were shown to significantly increase with time and subsequent SSRI treatment. It will be of interest to determine if O-mannosylation plays a role in calcium regulation by direct modification of SERCA2B.

\subsection{Summary}

The ER utilizes protein-protein interaction motifs to efficiently execute various roles to maintain cellular homeostasis in a large single luminal space and a contiguous membrane. In particular, the presence of TPR motifs provides binding specificity for subsets of interactors while domains outside of the TPRs mediate activity. Thus far, ER TPR- or TPR-like containing proteins contribute to protein translocation both into and out of the ER membrane, post-translational modification and act as co-chaperones. Eleven proteins, with tandem arrays from 2-10 sequential TPRs, have been identified that associate with or are localized within the ER. Interestingly, the presence of TPR motifs has become increasingly easier to predict using algorithms (Letunic and Bork, 2018), but the structures of the ER TPR-containing proteins are poorly understood, and oftentimes the function of the protein is identified prior to structure. Further studies using super resolution microscopy and proteomics are critical to provide a better understanding of the localization and identifying interacting partners of the ER TPR-containing proteins.

Given that the ER is involved in maintaining cellular protein, calcium and lipid homeostasis, it would be of interest to explore other families of repeat proteins, such as WD-40, ankyrin repeats or coiled-coil domains, to identify additional factors that 
contribute to ER morphology, organization and function. Coiled-coil domain containing proteins have previously been shown to contribute to ER membrane curvature and shape. The integral membrane protein Climp-63, which localizes exclusively in sheets, is thought to use its luminal coiled-coil domain to bridge between two apposed membranes (Klopfenstein et al., 2001). Additionally, the surface of ER sheets is kept flattened, likely by the sheet-enriched integral membrane proteins kinectin and $\mathrm{p} 180$, which possess a cytosolic coiled-coil domain that may form rod-like scaffolds (Shibata et al., 2010). WD40 repeat containing protein, Sec13p, is involved in ER cargo export and ankyrin repeat containing ASB11 is a novel ER-associated ubiquitin ligase (Andresen et al., 2014; Barlowe, 1998). There are also other protein domains, such as intrinsically disordered regions (IDRs) (i.e. in SEL1L, FICD and TMTC3) or Proline-rich regions (i.e. in SEL1L) predicted to be present in some of the ER TPR-containing proteins, which could contribute to essential interactions and function. Macromolecular complexes mediate many essential cellular processes. Key to understanding their mechanisms is knowing how these macromolecular assemblies are held together, localized and recruit substrates, properties that can be aided by critical repeat domains such as TPR motifs. 

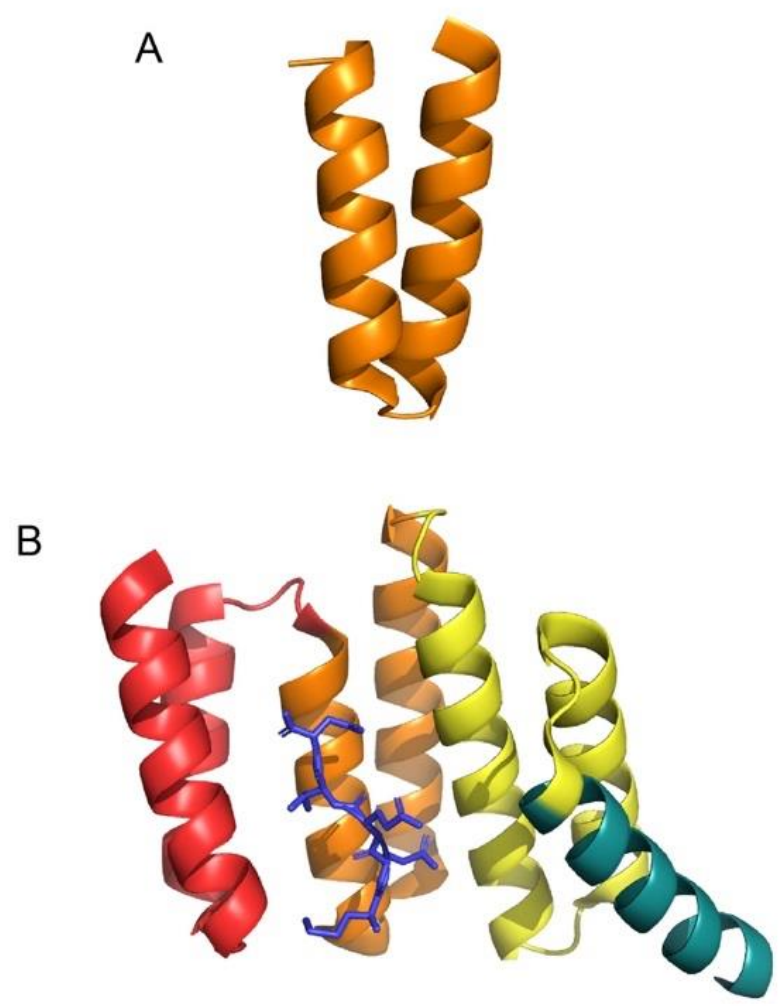

C

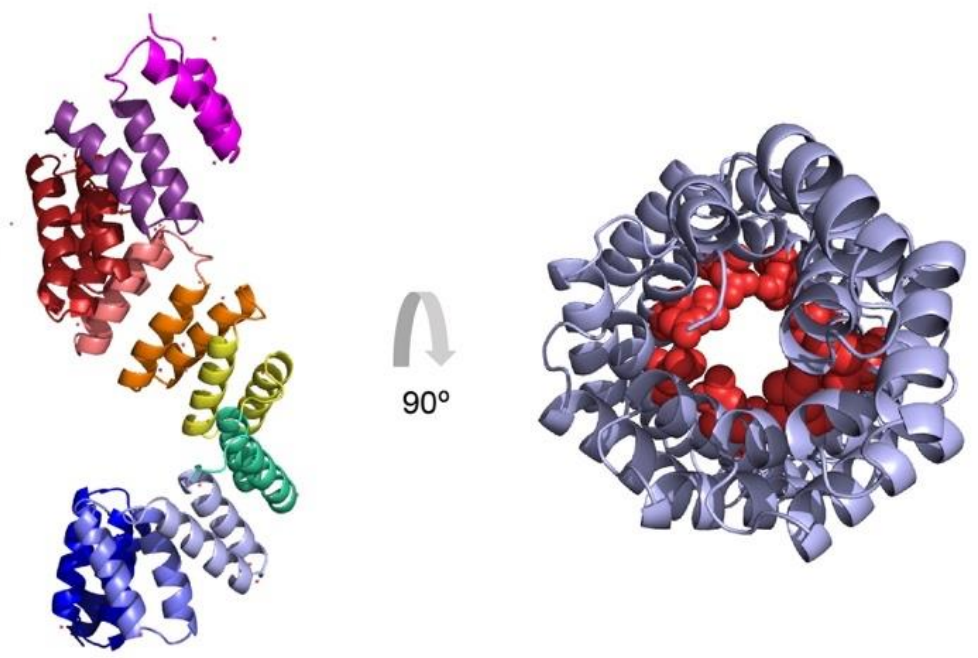

Figure 1.1. Tetratricopeptide Repeats. (A) A single tetratricopeptide repeat (TPR) motif (orange) consists of 34 amino acids that folds into two anti-parallel alpha helices. They 
are found in multiple organisms and all organelles within the cell. TPR motifs are known to mediate protein-protein interactions in multi-protein complexes and are most often found in clusters of 3 or more to form a functional domain. (B) The crystal structure (1ELR) represents the TPR domain found in Hsp70/Hsp90 Organizing Protein (HOP), which binds to the chaperone Hsp90. The single TPR motifs are indicated in red, orange and yellow, with the $\mathrm{C}$-terminal capping helix in turquoise. The $\mathrm{C}$-terminal pentamer of Hsp90, which is the ligand for this cluster, is shown in blue. (C) The crystal structure (1W3B) represents the TPR region of OGT. It forms a superhelix with individual motifs indicated in pink, purple, brick red, red, orange, yellow, green, periwinkle, blue and violet from $\mathrm{N}$-terminus to $\mathrm{C}$-terminus, respectively. The structure in the right panel is the 11.5 TPR motifs from left panel rotated 90 degrees, which demonstrates the superhelical nature of the tandem repeats. Conserved Asn (red spheres), thought to mediate target protein interaction, as seen in importin- $\alpha$, line the inner surface of the superhelix. 
SEL1L

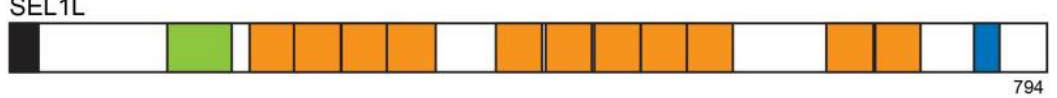

ERdj6

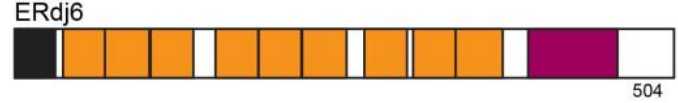

FICD

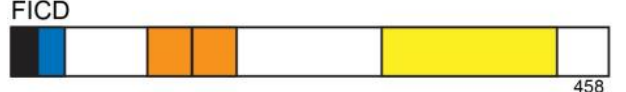

TMTC1
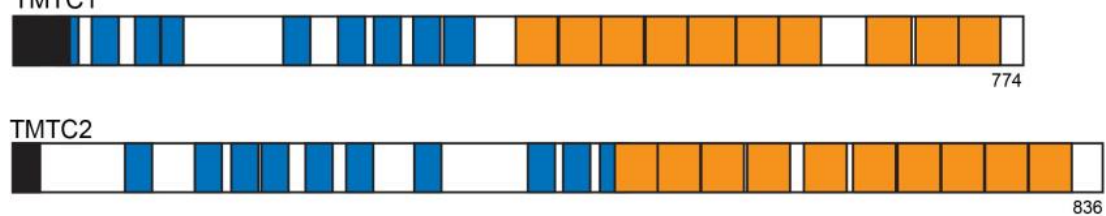

TMTC3

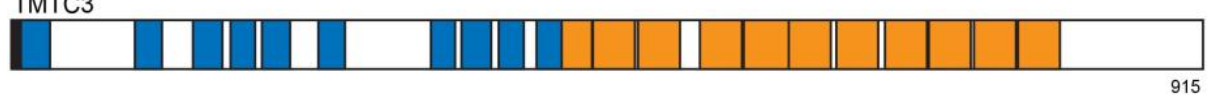

TMTC4

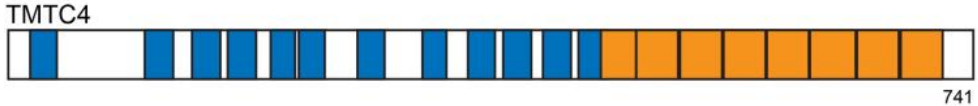

TTC13

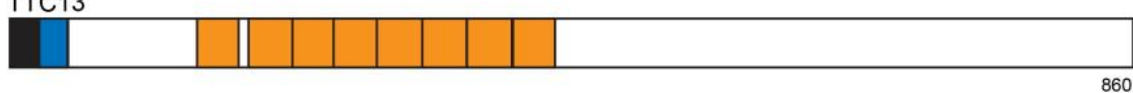

TTC17

860

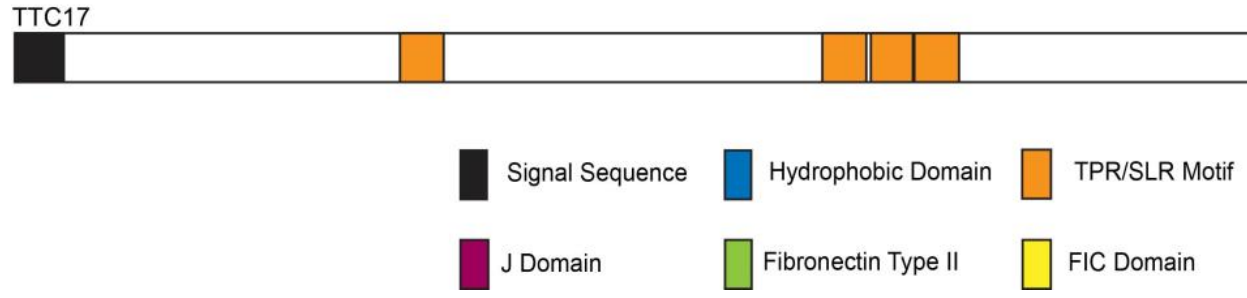

Figure 1.2. ER TPR-containing proteins. Nine TPR-containing proteins have been shown to localize to the ER. N-terminal signal sequences (black squares) target these proteins to the ER and the names, positions of tetratricopeptide repeats (TPRs) (orange squares) and hydrophobic domains (blue squares) are designated. SEL1L has a fibronectin-like type II domain at its N-terminus indicated in green. Erdj6's J-domain (magenta rectangle) and FICD's FIC domain (yellow rectangle) are located at the C-terminus of each protein. 

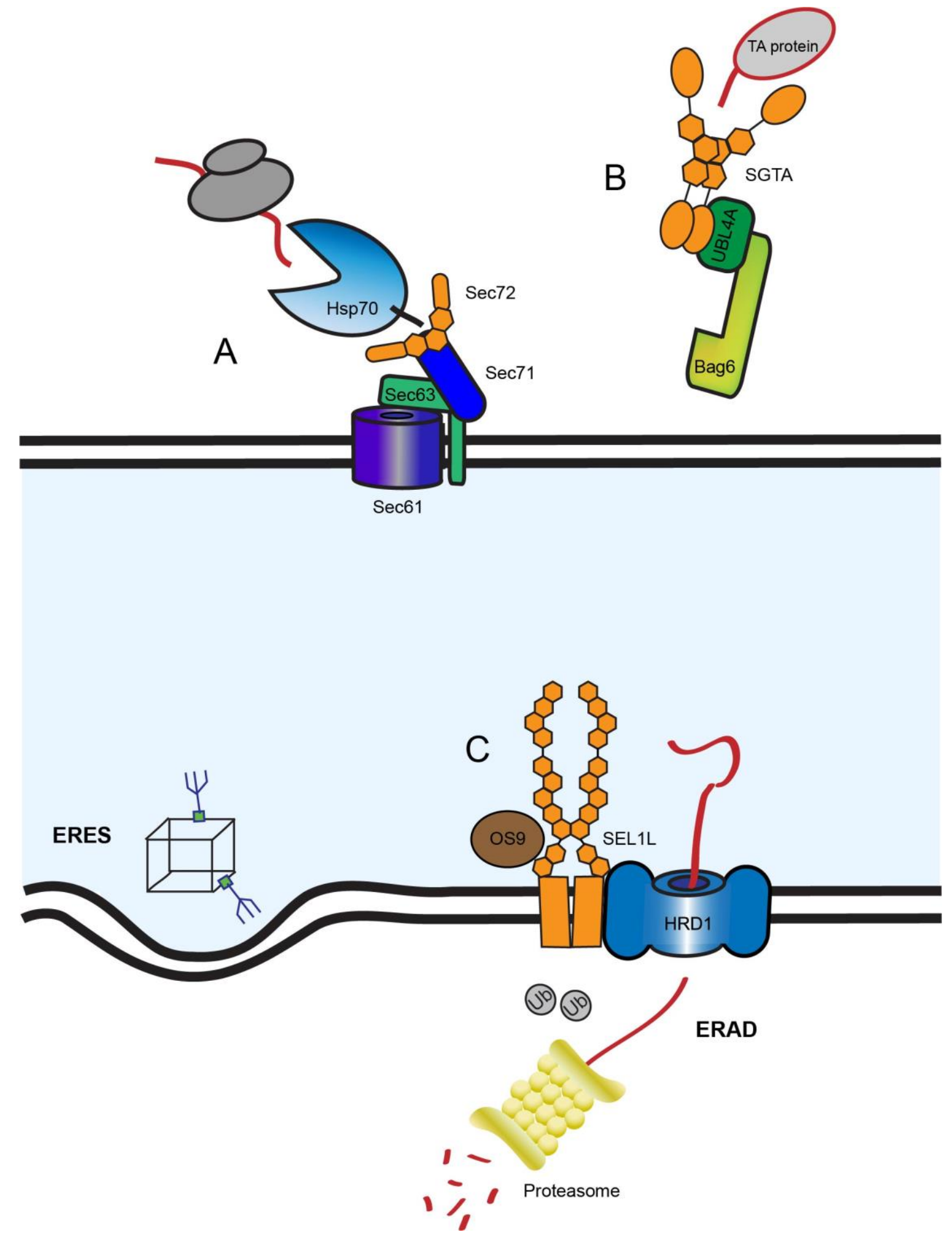

Figure 1.3. Sec72, SGTA and SEL1L are involved ER protein translocation. (A) Sec72 (orange) is anchored to the ER membrane by Sec71 (dark blue) and Sec63 (green) at the 
Sec61 (purple) translocon. The TPR domain, comprised of three TPR motifs (orange hexagons), binds the C-terminus of the yeast Hsp70 (blue), Ssa1, aiding in the recruitment of translated polypeptides to the Sec61 translocon. (B) SGTA (orange) aids in post-translational translocation, specifically of tail-anchored proteins. SGTA is comprised of an N-terminal UBL-binding domain, which is also responsible for homodimerization, a middle TPR domain and C-terminal substrate binding domain that captures the tail-anchored proteins (light grey). SGTA is in a complex with UBL4A and Bag6 (dark and light green, respectively) and plays a role in molecular triage. This complex is responsible for the proper targeting of tail-anchored proteins to the ER membrane as well as segregating misfolded polypeptides and targeting them for degradation. As chaperones aid in this process, SGTA's TPR domain is responsible for chaperone recruitment. The TPRs bind the C-termini of both Hsp70 and Hsp90 as well as proteasomal subunit, Rpn13. (C) As nascent polypeptides are queried for folding status, properly folded proteins are distinguished for export at ER exit sites (ERES) and misfolded proteins are targeted for degradation by ERAD. SEL1L (orange) is in complex with Hrd1, the ER retrotranslocon responsible for translocating misfolded proteins across the ER membrane for subsequent degradation by the proteasome (yellow) in the cytoplasm. SEL1L contains 11 SLR motifs (hexagon \#1-11 from N to C-terminus), which are very similar to TPR motifs but with an extended consensus sequence length, that comprise the majority of the luminal portion of the protein. The SLR motifs nearest the membrane (\#10-11) interact with a luminal portion of Hrd1 and are necessary for interaction with ERAD factor, OS9 (brown). A TPR motif in the middle region (\#9) is responsible for homo-oligomerization of SEL1L. 


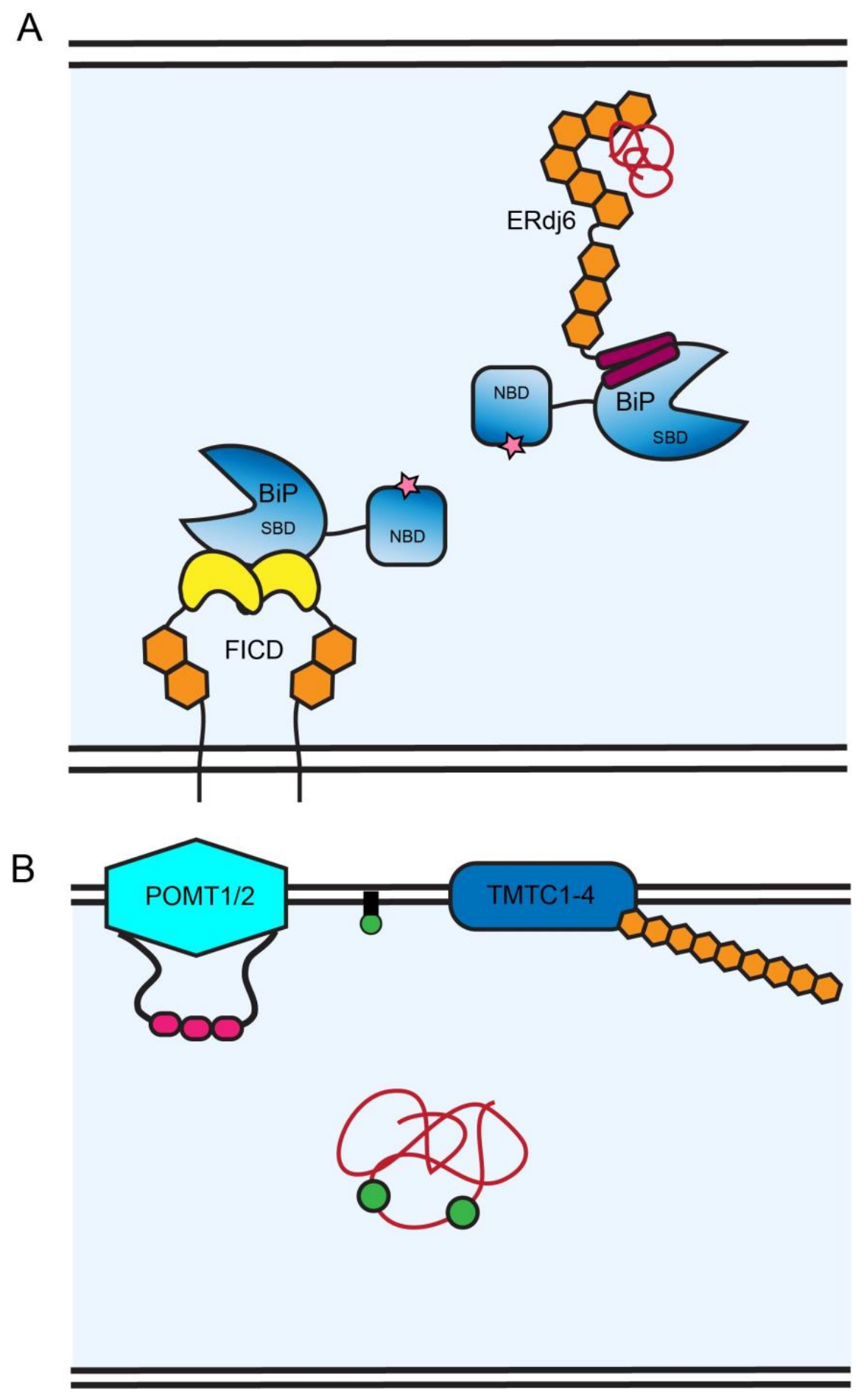

Figure 1.4. ER TPR co-chaperone, Erdj6, and protein modifiers, FICD and TMTC1-4 control protein homeostasis through interactions with BiP and glycosylation. (A) Erdj6 has 9 TPR motifs (orange hexagons) followed by a C-terminal J domain (magenta 
rectangles). The J-domain contains an Hsp70 interaction motif, HPD, and modulates the nucleotide binding activity of the ER Hsp70, BiP (gradient blue). The N-terminal TPR domain of Erdj6 can bind exposed hydrophobics on folding polypeptides (red) and potentially pass them off or sequester them for BiP bound at the J-domain. FICD is comprised of $2 \mathrm{~N}$-terminal TPR motifs (orange hexagons) and a C-terminal FIC domain (yellow), which is responsible for regulating BiP chaperone activity by AMPylating BiP on its substrate binding domain. FICD forms a homodimer mediated through the FIC domain and AMPylates BiP under normal conditions to create an inactive $\mathrm{BiP}$ pool. As the UPR is activated, FICD de-AMPylates BiP so that it may engage unfolded substrate (red). (B) TMTC1-4 (blue and orange) are implicated in O-mannosylation. The TMTCs are composed of $\mathrm{N}$-terminal hydrophobic domains (blue) embedding them in the ER membrane and 8-10 consecutive C-terminal TPR motifs (orange hexagons). POMT1 and 2 are the known protein O-mannosyl transferases of the ER. They are composed of a number of transmembrane domains represented by the light blue hexagon and contain 3 MIR domains (pink) in a luminal loop. The MIR domains are thought to recruit substrate to the membrane so that O-mannosyl transferases can transfer a mannose (green circle) from the dolichol-mannose precurser (black and green) in the membrane to the substrate (red). 


\section{CHAPTER 2}

\section{TMTC3 CONTRIBUTES TO O-MANNOSYLATION OF E-CADHERIN}

\subsection{Abstract}

Protein glycosylation plays essential roles in protein structure, stability and activity such as cell adhesion. The cadherin superfamily of adhesion molecules carry Olinked mannose glycans at conserved sites and it was recently demonstrated that the TMTC1-4 genes contribute to the addition of these O-linked mannoses. Here, biochemical, cell biological and organismal analysis was used to determine that TMTC3 supports the O-mannosylation of E-cadherin, cellular adhesion and embryonic gastrulation. Using genetically engineered cells lacking all four TMTC genes, overexpression of TMTC3 rescued O-linked glycosylation of E-cadherin and cell adherence. Knockdown of the Tmtcs in Xenopus laevis embryos caused a delay in gastrulation that was rescued by the addition of human TMTC3. Mutations in TMTC3 have been linked to neuronal cell migration diseases including Cobblestone lissencephaly. Analysis of TMTC3 mutations associated with Cobblestone lissencephaly found that three of the variants exhibit reduced stability and missence mutations were unable to complement TMTC3 rescue of gastrulation in Xenopus embryo development. Our study demonstrates that TMTC3 regulates O-linked glycosylation and cadherinmediated adherence, providing insight into its effect on cellular adherence and migration, as well the basis of TMTC3-associated Cobblestone lissencephaly.

\subsection{Introduction}

Protein glycosylation is the most common and diverse co/post-translational protein modification (Freeze and Elbein, 2009). Carbohydrates play general metabolic, 
structural and biophysical roles in the cell (Apweiler et al., 1999; Hebert et al., 2014; O’Connor and Imperiali, 1996; Price et al., 2012; Solá and Griebenow, 2009; Varki and Sharon, 2009). There are two general types of glycosylation that are categorized by the type of bond used to covalently attach the carbohydrate to the protein (Imperiali and Hendrickson, 1995; Jayaprakash and Surolia, 2017). N- and O-linked glycans are attached to proteins through $\mathrm{N}$-glycosidic (Asn) or O-glycosidic (Ser/Thr) bonds, respectively. A third of the proteome is targeted to the secretory pathway in eukaryotic cells where both $\mathrm{N}$ - and O-linked modifications are commonly observed. The endoplasmic reticulum (ER) serves as the entry portal for secretory pathway cargo. The majority of the proteins in the ER are modified by N-linked glycans; however, a restricted fraction is modified by O-linked glycans (Apweiler et al., 1999; VesterChristensen et al., 2013).

A diverse set of O-glycans are added in the ER that include mannose, fucose, glucose or N-acetyl glucosamine (Joshi et al., 2018). O-mannosylation is conserved from fungi to mammals. It is initiated by the transfer of a mannose saccharide from a donor dolichol P-mannose substrate embedded in the ER membrane to a Ser/Thr on the target protein (Bause and Lehle, 1979). This transfer is catalyzed by ER resident protein Omannosyl transferases most commonly involving POMT1 and POMT2 with $\alpha$ dystroglycan as the main substrate in mammals, and Pmt1-7 in yeast (Guerreiro et al., 1996; Immervoll et al., 1995; Lussier et al., 1995; Strahl-Bolsinger et al., 1993; Wing et al., 1992). Their general architecture is that of large polytopic membrane proteins, which display variations of a diacidic motif (i.e. Asp-Glu) in the first luminal loop region proximal to the membrane (Figure 2.4B) (Bai et al., 2019; Lairson et al., 2008; Liu and 
Mushegian, 2003; Loibl and Strahl, 2013). Metazoan and yeast O-mannosyltransferases also contain conserved Mannosyltransferase, Inositol triphosphate and Ryanodine receptor (MIR) domains. MIR domains have been shown to be essential for enzymatic activity in yeast and have been implicated in substrate binding based on studies performed on other mammalian MIR domain containing proteins such as SDF2 (Fujimori et al., 2017; Girrbach et al., 2000; Lommel et al., 2011; Meunier et al., 2002; Ponting, 2000).

A new putative family of O-mannosyl transferases was recently discovered in mammals (Larsen et al., 2017a, 2017b). This family is comprised of four tetratricopeptide repeat (TPR)-containing proteins that appear to be ER polytopic transmembrane proteins called TMTC1-4 (transmembrane and TPR-containing proteins 1-4)(Cao et al., 2012; Della-Morte et al., 2011; Larsen et al., 2017a; Li et al., 2018; Racapé et al., 2011; Sunryd et al., 2014). TMTC1, 2 and 4 are ER proteins involved in $\mathrm{Ca}_{2}+$ regulation as they associate with ER Ca2+ re-uptake pump SERCA2B (Li et al., 2018; Sunryd et al., 2014). The knockouts of TMTC1-4 have been shown using glycoproteomics to be involved in the O-mannosylation of cadherins (Larsen et al., 2017a, 2019). TMTC1-4 contain homologous structural elements as their $\mathrm{N}$-terminal halves are comprised of a number of hydrophobic domains and the $\mathrm{C}$-terminal halves are predicted to be composed mainly of long stretches of TPR motifs (8-12) (Figure 2.1A) (Karpenahalli et al., 2007; Letunic and Bork, 2018). Two of the TMTCs, TMTC3 and TMTC4, contain putative diacidic motifs (Asp-Asp or Glu-Glu) on N-terminal luminal loops based on predictive topology, making them appear homologous to the POMTs and Pmts (Figure 2.4B) (Bai et al., 2019; Hessa et al., 2007). 
TPRs are protein structural motifs that support protein-protein interactions and are frequently found on co-chaperones (Brehme et al., 2014; Magliery and Regan, 2005). A single TPR motif consists of a degenerate 34-amino acid sequence that is comprised of two anti-parallel $\alpha$-helices (Figure 1.1A) (D’Andrea and Regan, 2003; Magliery and Regan, 2005). TPR domains are most commonly found in a cluster of three TPR motifs; however, clusters comprised of up to sixteen sequential TPRs have been observed (D’Andrea and Regan, 2003). Proteins with three TPRs in a cluster favor the recognition of short and defined sequences, whereas proteins with long stretches of consecutive TPRs tend to be more promiscuous in their selectivity. Unlike classic TPR co-chaperones, which contain three to four consecutive motifs, the composition of TMTC1-4, with eight to twelve consecutive motifs, is similar to that of the well-studied TPR-containing cytoplasmic O-GlcNAc transferase (OGT), which contains a stretch of 12.5 TPRs followed by a C-terminal catalytic domain (Jínek et al., 2004; Lazarus et al., 2011). The structure of OGT reveals that the TPRs form a super helix responsible for homodimerization of OGT and are proposed to scaffold interactions with other proteins, potentially playing a role in substrate selectivity (Figure 1.1C). Dimerization of the TPR domain is mediated by the convex faces of the superhelical monomers, and the concave surface contains conserved Asn, which form a continuous ladder (Figure 1.1C right panel). This Asn conservation is similarly observed in the ARM-repeat proteins importin$\alpha$ and $\beta$-catenin (Conti et al., 1998; Huber and Weis, 2001). The Asn in these proteins contribute to binding of a target peptide (nuclear localization sequence with importin- $\alpha$ and E-cadherin with $\beta$-catenin), suggesting that the TMTCs may use a similar mechanism of protein-protein interaction and substrate selection. 
Mutations in the TMTC genes have been linked to various human disease states (Farhan et al., 2017; Jerber et al., 2016; Li et al., 2018; Runge et al., 2016). Point mutations in human TMTC2 and the knockout of Tmtc4 in mice result in hearing loss (Guillen-Ahlers et al., 2018; Li et al., 2018; Runge et al., 2016). Ten mutations in TMTC3 are associated with neuronal cell migration diseases (Farhan et al., 2017; Jerber et al., 2016). Biallelic mutations resulting in single amino acid or frame shift changes in TMTC3 were identified in a cohort of families with recessive forms of Cobblestone lissencephaly, a severe brain malformation in which over migration of neurons and glial cells results in the formation of cortical dysplasia, or brain development abnormalities (Jerber et al., 2016). Another study identified heterozygous variants of TMTC3 in patients with periventricular nodular heterotopia $(\mathrm{PVNH})$, a common brain malformation caused by the failure of neurons to migrate from the ventricular zone to the cortex (Farhan et al., 2017). While mutation or loss of the TMTC genes has been associated with a number of diseases, an understanding of how these mutations result in specific defects is unclear.

Here, in silico, biochemical, cell and developmental biological approaches were used to expand our understanding of the organization, localization, activity and function of TMTC3 and TMTC4. Previously uncharacterized TMTC3 and 4 were identified as ER TPR-containing membrane proteins with their TPR domains orientated within the ER lumen. Using TMTC HEK293 knockout cells, it was demonstrated that TMTC3 complementation recovered the O-mannosylation of E-cadherin. While the knockout of the TMTCs did not affect the stability, localization or trafficking of E-cadherin, it did affect cellular adherence and the overexpression of TMTC3 was able to partially recover 
adherence, specifically E-cadherin mediated adherence. Subsequent analysis of protein O-mannosyltransferase protein architecture showed that TMTC3 shares functional similarity with O-mannosyltransferases (POMT1/2 and Pmt1-7) as it contains two diacidic motifs (Asp-Asp and Glu-Glu) located in a putative N-terminal luminal loop and mutation of the Asp-Asp motif results in reduced TMTC3 stability. Additionally, the knockdown of Tmtc3 and Tmtc4 in Xenopus laevis resulted in an embryonic gastrulation delay phenotype and the delay was rescued by human TMTC3. There are eight disease variants of TMTC3 recently associated with Cobblestone lissencephaly and two associated with PVNH (Farhan et al., 2017; Jerber et al., 2016). While novel biochemical characterization of eight of these disease variants showed that they all localize to the ER, three of them are less stable than wild type (WT) and none of missense mutations are able to rescue the delay in gastrulation caused by the knockdown of Tmtc3 in Xenopus laevis. The identification of TMTC3's role in O-glycosylation of E-cadherin in combination with its knockdown in Xenopus embryos provides further insight into the role Oglycosylation plays in cell-cell adhesion and migration, and the etiology of Cobblestone lissencephaly caused by TMTC3 mutation.

\subsection{Results}

\subsubsection{TMTC3 and TMTC4 are ER resident proteins.}

In silico analysis, using SignalP4.0, TargetP1.1, $\Delta \mathrm{G}$, TPRPred and domain architecture database SMART7, indicated that TMTC3 (NCBI Accession \# NP_861448.2. (http://www.ncbi.nlm.nih.gov/protein/NP_861448.2)) and TMTC4 (NCBI Accession \# NP_001073137.1 (http://www.ncbi.nlm.nih.gov/protein/NP_001073137)) contained potential $\mathrm{N}$-terminal signal sequences, ten and twelve hydrophobic segments 
and eleven and eight C-terminal TPR motifs, respectively (Figure 2.1A) (Emanuelsson et al., 2000; Hessa et al., 2007; Karpenahalli et al., 2007; Letunic and Bork, 2018; Nielsen, 2017; Nielsen et al., 1997, 2019). TMTC3 and TMTC4 cDNAs were subcloned into mammalian expression vectors encoding a C-terminal S-tag, and their cellular localization was determined by glycosylation assay and confocal immunofluorescence microscopy. Secretory proteins are commonly modified in the ER with $\mathrm{N}$-linked glycans at the consensus site Asn-Xxx-Ser/Thr. TMTC3 and TMTC4 possess five and three predicted N-linked glycosylation consensus sites, respectively (Figure 2.1A), therefore a glycosylation assay was used to further analyze ER targeting and localization (Gupta, 2002). As the molecular weight of an $\mathrm{N}$-linked glycan is $\sim 2.5 \mathrm{kDa}$, the removal of $\mathrm{N}$ linked glycans by glycosidase treatment results in a corresponding increase in mobility for the deglycosylated protein. Endoglycosidase H (Endo H) trims the high mannose glycans encountered in the ER while Peptide-N-Glycosidase F (PNGase F) removes complex glycans acquired in the Golgi in addition to high mannose glycans.

HEK293T cells were transfected with TMTC3 or TMTC4 containing C-terminal S-tags. Cell lysate and media fractions were affinity precipitated with S-protein agarose beads followed by glycosidase treatment. Shifts upon PNGaseF treatment (Figure 2.1B, lanes 9 and 15) were observed for both TMTC 3 and TMTC4 demonstrating that both proteins were targeted to the ER and received N-linked glycans. A similar increase in mobility was observed upon Endo $\mathrm{H}$ treatment (Figure 2.1B, lanes 8 and 14) indicating that the carbohydrates were high mannose glycoforms suggesting that TMTC3 and TMTC4 are ER resident proteins (Figure 2.1B). 
COS7 cells were transfected with either TMTC3 S-tag or TMTC4 S-tag as COS7 cells are highly amenable to imaging. Immunofluorescence staining of TMTC3 and TMTC4 was compared against an ER (ERp57) or Golgi (GM130) marker (Figure 2.1C). Both TMTC3 and TMTC4 co-localized with Erp57, while co-localization was not observed with GM130 (Figure 2.1C). Therefore, the glycosylation profiles for TMTC3 and TMTC4 and the cellular distribution are consistent with TMTC3 and TMTC4 residing in the ER.

\subsubsection{TMTC3 and TMTC4 are ER membrane proteins with luminal orientated TPR motifs.}

Analysis of the TMTC 3 and TMTC4 protein sequences with $\Delta \mathrm{G}$ prediction demonstrated that they contained ten and twelve hydrophobic segments, respectively, that could potentially serve as transmembrane domains to create polytopic membrane proteins (Figure 2.1A) (Hessa et al., 2007). Alkaline extraction of membrane fractions was performed to separate membrane and soluble forms of proteins following centrifugation to determine if TMTC 3 and TMTC4 are integral membrane proteins (Mostov et al., 1981).

HEK293T cells were transfected with either TMTC3 or TMTC4 S-tag, and proteins were radiolabeled with [35S]-Met/Cys for $1 \mathrm{hr}$. Cells were homogenized in isotonic buffer and fractions were separated by centrifugations. TMTC 3 and TMTC4 were found in the nuclear $(\mathrm{N})$ and total membrane (TM) fractions (Figure 2.2A, lanes 2 and 4). The nuclear localization of TMTC3 and TMTC4 is likely explained by the contiguous nature of the ER and nuclear membrane prohibiting their separation as the ER proteins calnexin and calreticulin were also in the nuclear fractions. Alkaline extraction 
of the total membrane fractions followed by centrifugation found TMTC 3 and TMTC4 exclusively in the membrane pellet (P) (Figure 2.2A, lane 6). This profile was observed for the ER membrane protein, calnexin, and not its soluble paralogue calreticulin, which largely accumulated in the supernatant (S) (Figure 2.2A, lane 5). Therefore, both TMTC3 and TMTC4 are integral membrane proteins.

Since TMTC3 and TMTC4 are membrane proteins, a trypsin protection assay was employed to determine if their C-terminal TPR motifs are positioned in the ER lumen or the cytoplasm. HEK293T cells were transfected with either TMTC3 or TMTC4 constructs containing C-terminal S-tags, and cells were homogenized prior to isolation of ER-enriched microsomes. Isolated microsomes were resuspended in an isotonic buffer, and aliquots were treated with triton X-100 and trypsin as indicated. Trypsin treatment produced a discrete TMTC3 fragment of $\sim 83.5 \mathrm{kDa}$ and TMTC4 fragments of $\sim 67.8$ and $57.1 \mathrm{kDa}$ (Figure 2.2B, compare lanes 5 to 6 and 9 to 10). As the TPR domains and Stags are both at the C-termini, this demonstrated that the TPR domains of TMTC3 and TMTC4 were positioned in the ER lumen (Figure 2.2C). Combined with the modification of the glycosylation sites added to the TPR rich regions (Figure 2.1B), these results demonstrated that the TPR motifs for both of the membrane proteins, TMTC3 and TMTC4, were facing the ER lumen.

\subsubsection{TMTC3 and TMTC4 are upregulated by folding and trafficking stress.}

To compare TMTC3 and 4 to known O-mannosyltransferases, the basal mRNA levels of the two classes of proteins were examined. TMTC1-4 and POMT1/2 mRNAs were similarly expressed with TMTC4 and POMT1 showing the highest levels at $\sim 2.5 \%$ of the reference gene $\beta$-actin (Figure 2.3A). All TMTCs and POMTs were significantly 
less expressed than the ER resident chaperone, BiP, which is $\sim 20 \%$ of $\beta$-actin (Figure 2.3A).

Proteins that reside in the secretory pathway are frequently transcriptionally upregulated by stress (Walter and Ron, 2011). To determine whether the TMTC3 and TMTC4 genes are transcriptionally regulated by ER stress, HEK293A cells were exposed to different ER stress conditions. Cells were subjected to $\mathrm{N}$-glycan synthesis inhibition (tunicamycin), calcium depletion (thapsigargin), redox stress (dithiotreitol, DTT), inhibition of anterograde protein trafficking (brefeldin A) or proteasomal inhibition (MG132). RNA was harvested from cells followed by reverse transcription to generate cDNA and changes in gene expression were measured by qRT-PCR.

TMTC3 gene expression was increased with thapsigargin and brefeldin A treatment by $\sim 2.1$ and 2.5-fold, respectively (Figure 2.3B). TMTC4 gene expression was increased with tunicamycin treatment by 1.8 -fold and 1.5 -fold with brefeldin A treatment (Figure 2.3B). Thapsigargin, DTT and MG132 did not produce a significant increase in gene expression of $T M T C 3$, although the transcription of $B i P$ was stimulated by all treatments. Interestingly, POMT1 and 2 were up-regulated by a number of stresses, including a 2-fold increase by tunicamycin (POMT1), and a 2- and 2.5-fold increase by thapsigargin for POMT1 and 2, respectively. Both POMT1 and 2 were up-regulated by brefeldin A treatment, 2.3 and 2.8-fold, respectively. POMT1 was also up-regulated 1.4fold by redox stress. Out of the TMTCs, TMTC4 was up-regulated by stress most significantly and POMT1 exhibited similar up-regulation in response to stress, which may correspond to their basal mRNA levels being higher or UPR induction upon their respective deletion (Jonikas et al., 2009; Li et al., 2018). 


\subsubsection{TMTC3 rescues O-mannosylation of E-cadherin}

TMTC1, TMTC2 and TMTC4 have been shown to associate with SERCA2B and play a role in calcium regulation (Li et al., 2018; Sunryd et al., 2014). To investigate the cellular role of TMTC3, initially its binding to SERCA2B was tested. HEK293T cells were transfected with either TMTC1, TMTC2, TMTC3 or TMTC4 cDNA. Upon cell homogenization and total membrane isolation, the TMTCs were affinity purified, and associated proteins were resolved by SDS-PAGE. Binding to SERCA2B was monitored by immunoblotting (Figure 2.5A). As previously shown, TMTC1, TMTC2 and TMTC4 associated with SERCA2B; however, no interaction between TMTC3 and SERCA2B was observed (Figure 2.5A, lane 9). Therefore, unlike the other TMTCs, TMTC3 does not appear to associate with SERCA2B or be involved in calcium regulation.

TMTC1-4 were discovered to contribute to the O-mannosylation proteome when a subset of substrates remained O-mannosylated in cells that lacked the known Omannosyltransferases, POMT1 and POMT2 (Larsen et al., 2017a, 2017b). A significant difference in O-mannosylation of the cadherin family of proteins was observed upon knockout of all four TMTCs in HEK293 cells (HEK293 quadruple knockout cells (TMTC1/2/3/4-/-)) (Larsen et al., 2017a). Glycoproteomics was used to determine that Ecadherin was O-mannosylated at nine sites (Figure 2.4A). These results were obtained using HEK293 cells also lacking COSMC and POMGnT1. COSMC is an essential chaperone to T-synthase that modifies glycoproteins with an O-GalNAc linked saccharide, and POMGnT1 is a glycosyltransferase that appends $\beta 1,2-$ GlcNAc to Omannose in the Golgi (Wang et al., 2010; Yoshida et al., 2001). The use of 
COSMC/POMGnT1-/- cells created truncated, more homogenous O-mannosylated modifications amenable for glycoproteomics analysis (Vester-Christensen et al., 2013).

To assess potential O-mannosyl transferase activity of the individual TMTCs, a glycosylation and carbohydrate binding assay was employed in glycosyltransferase deficient cell lines including COSMC/POMGnT1/TMTC1/2/3/4--. HEK293 cells with TMTCs complementation (Figure 2.5B). These cells were transfected at similar levels individually with S-tagged TMTC1, 2, 3 or 4 (Figure 2.13A). Cell lysates were collected and divided into two fractions. One fraction of the lysate was treated with PNGaseF to remove $\mathrm{N}$-linked glycans (Figure 2.5B, lane 2) prior to both fractions being affinity purified using the lectin, concanavalin A (Con A). Samples were analyzed via SDSPAGE and immunoblotted with E-cadherin antisera.

E-cadherin levels were not affected by the deletion of COSMC or the glycosyltransferases (Figure 2.6A). E-cadherin appears to receive O-linked glycans as it was pulled down by Con A even though the glycoproteins lacked N-linked glycans in the PNGaseF treated sample (Figure 2.5B, top blot, lane 2). Subsequently, the same assay was performed in HEK293 COSMC/POMGnT1/POMT1/POMT2-- cells and Oglycosylated E-cadherin still bound to Con A (Figure 2.5B, lane 2, middle blot). However, in HEK293 COSMC/POMGnT1/TMTC1/2/3/4-/- cells, O-glycosylated Ecadherin was not recovered by Con A after N-linked glycan removal suggesting a significant loss of both $\mathrm{N}$ - and O-linked glycans (Figure 2.5B, lane 2, bottom blot). This result was similarly observed in quadruple $T M T C$ s knockout cells transfected with Stagged TMTC1, 2 or 4 (Figure 2.5B, lanes 4, 6 and 10, bottom blot). However, cells transfected with TMTC3 were able to recover Con A-bound O-linked glycosylated E- 
cadherin (Figure 2.5B, lane 8, bottom blot). The recovery of O-glycosylated E-cadherin by TMTC 3 expression was repeated and quantified to confirm that TMTC 3 contributed to the O-linked glycosylation of E-cadherin (Figure 2.5C).

To determine whether TMTC3 mediated O-linked glycosylation of E-cadherin included O-mannosylation, a combinatorial endoglycosidase assay was performed. Stagged TMTC3 was transfected into HEK293 COSMC/POMGnT1/TMTC1/2/3/4--- cells and the lysate was subsequently treated with PNGaseF or both PNGaseF and $\alpha 1-2,3,6$ mannosidase prior to affinity purification with Con $\mathrm{A}$, as the mannosidase would specifically remove mannose residues. O-glycosylated E-cadherin was recovered from cells complemented with TMTC3 after PNGaseF treatment; however, the amount bound to Con A upon subsequent $\alpha 1-2,3,6$ mannosidase treatment was reduced by $\sim 45 \%$, indicating that TMTC3 contributes to O-mannosylation of E-cadherin (Figure 2.5D insert, compare lanes 3 to 2 ).

\subsubsection{TMTC3 enhances cellular adherence and binding to E-cadherin.}

Studies on protein O-mannosylation in yeast have revealed that O-glycans are important for protein stability, trafficking, localization, function and stress response (Petkova et al., 2012; Xu and Ng, 2015; Yang et al., 2009). To understand the effect of TMTC3 on E-cadherin, the expression, stability and trafficking of E-cadherin was assessed in TMTC1-4 knockout cells. Briefly, HEK293 COSMC/POMGnT1-/, COSMC/POMGnT1/POMT1/2-- and COSMC/POMGnT1/TMTC1/2/3/4-ı- cells were lysed and subjected to lectin affinity purification with Con A prior to samples being analyzed via SDS-PAGE and immunoblotted with E-cadherin antisera. There was no significant difference observed in expression between cell types (Figure 2.6A). Stability 
of E-cadherin was also assessed by a cycloheximide chase assay and a significant difference was not observed (Figure 2.6B). Cell surface localization of several cadherins, including E-cadherin, was monitored using confocal immunofluorescence microscopy. HEK293 COSMC/POMGnT1--, COSMC/POMGnT1/POMT1/2-/- and COSMC/POMGnT1/TMTC1/2/3/4-/- cells were fixed and stained for pan-cadherin (Figure 2.7A). In all three cell lines, cadherins localized to the cell surface. Interestingly, a morphology defect was observed in cells lacking TMTC1-4. While cell surface staining in HEK293 COSMC/POMGnT1-- and COSMC/POMGnT1/POMT1/2-/- cells appeared round and evenly distributed, cadherin staining in HEK293

COSMC/POMGnT1/TMTC1/2/3/4-/- cells showed greater irregularity.

Cell-cell adhesion interactions are essential for embryogenesis, tissue morphogenesis and renewal (Halbleib and Nelson, 2006). Adherins junctions are the sites of cell-cell contact where adhesion is mediated by cell-surface receptors of the cadherin family (Meng and Takeichi, 2009). E-cadherin plays a critical role in cell-cell adhesion and development and O-mannosylation has been shown to be important for its role in forming adherens junctions (Lommel et al., 2013). To determine the effect of TMTCs on E-cadherin mediated cellular adherence, general cellular adherence was evaluated. HEK293 COSMC/POMGnT1--, COSMC/POMGnT1/POMT1/2-/- and COSMC/POMGnT1/TMTC1/2/3/4-/- cells were re-suspended in growth media and subsequently plated and allowed to adhere for $30 \mathrm{~min}$. After $30 \mathrm{~min}$, the floating cells were collected for each cell type and adhered cells were trypsinized and collected. Each fraction was quantified and the percent of floating cells over the total was calculated. HEK293 COSMC/POMGnT1-/ and COSMC/POMGnT1/POMT1/2-/- cells showed 
similar levels of adherence with average floating cell percentages at 23\% (Figure 2.7B). Contrastingly, HEK293 COSMC/POMGnT1/TMTC1/2/3/4--- cells demonstrated reduced cellular adherence as the percentage of floating cells observed was $~ 33 \%$ (Figure 2.7B). To determine if any one of the TMTCs individually contributed to cellular adherence, TMTC1, 2, 3 and 4 cDNA was transfected into HEK293 COSMC/POMGnT1/TMTC1/2/3/4-/- cells. The adherence assay described above was performed and the percentage of adhered cells as compared to mock was calculated. Upon establishing a baseline using the mock treated cells, TMTC3 transfected cells were able to recover adherence the most with an observed increase over mock of $\sim 8 \%$ (Figure 2.7C).

Cadherins are cell-surface membrane glycoproteins that contain multiple repeats of extracellular cadherin (EC) domains and they mediate cell-cell adhesion by trans homodimerization between apposed cells (Brasch et al., 2012; Cavallaro and Christofori, 2004; Yagi, 2008). E-cadherin mediated cell-cell adhesion was assessed in HEK293 COSMC/POMGnT1--, COSMC/POMGnT1/POMT1/2--- and COSMC/POMGnT1/TMTC1/2/3/4-/- cells to determine if genetic manipulation of the glycosyltransferases affected its adhesion function. E-cadherin substrate was incubated overnight to allow for attachment to the plate surface. HEK293 COSMC/POMGnT1--, COSMC/POMGnT1/POMT1/2--- and COSMC/POMGnT1/TMTC1/2/3/4--- cells were then resuspended and $\sim 60,000$ cells were seeded on the substrate. Cells were allowed to adhere for $1 \mathrm{hr}$ prior to discarding unadhered cells. Adhered cells were then fixed with paraformaldehyde before permeabilization with triton X-100, and nuclei were stained with Hoescht. Hoescht images were captured at five different locations within each well 
and the number of nuclei quantified. COSMC/POMGnT1/TMTC1/2/3/4-/- cells showed markedly reduced binding to the extracellular region of E-cadherin compared to COSMC/POMGnT1-/- and COSMC/POMGnT1/POMT1/2-/- cells (Figure 2.7D and E). The same assay was performed on COSMC/POMGnT1/TMTC1/2/3/4-/- cells expressing TMTC3 and E-cadherin mediated adherence was recovered. These results, combined with the results from the carbohydrate binding assay (Figure 2.5B-D), suggest that TMTC3 affects E-cadherin mediated adherence through O-mannosylation of the glycoprotein.

\subsubsection{TMTC3 disease variants exhibit reduced stability.}

Cell-cell adhesion interactions are essential for embryogenesis, tissue morphogenesis and renewal, all key processes of development (Halbleib and Nelson, 2006). Cobblestone lissencephaly is a severe brain malformation characterized by irregular borders, dysplasia, hypoplasia and dysmyelination, which are due to overmigration of neurons and glial cells beyond the external basement membrane. The cause of this over migration defect is impaired interactions between glial limitans and the extracellular matrix of the basement membrane (Barkovich et al., 2012; Siegenthaler and Pleasure, 2011). Glycosylated cell surface binding receptors provide a physical link between the cytoskeleton of the glial cells and the basement membrane (Barresi and Campbell, 2006). Loss of glycosylation of these molecules contributes to functional defects during development by reducing binding to the extracellular matrix (Michele et al., 2002). Cobblestone lissencephaly has been associated with mutations in sixteen genes, and twelve of these genes are involved in O-linked glycosylation, including POMT1 and 2 (Jerber et al., 2016). 
Recently, a study identified biallelic mutations in TMTC3 from Cobblestone lissencephaly patients exhibiting intellectual disability, hypotonia and delayed milestones (Jerber et al., 2016). Six families had a total of eight distinct mutations in affected individuals. Using predictive algorithms, the altered organization of seven of these mutants and a heterozygous PVNH-associated mutant $(\mathrm{R} 71 \mathrm{H})$ was analyzed (Figure 2.8A). The point mutations do not appear to alter organization; however, the resulting residue mutations change the charge of the native residue, which may have some topological or functional effect on TMTC3 as some of these mutations are in close proximity to the putative active sites (Figure 2.8A, H67D, R71H and G384E). The frame shift mutations beginning at residue positions 488,562 and 654 greatly change the number and organization of the predicted TPR motifs resulting in truncated forms of TMTC3. Additionally, these frameshift mutations could result in five to seven non-native amino acids being transcribed before the premature stop. The frameshift mutation at position 841 results in a shortened C-terminus and three putative non-native residues, and the early stop codon mutation at position 873 would result in a protein that is missing 41 amino acids.

TMTC3 disease variants were characterized for ER localization via glycosidase assay and immunofluorescence confocal microscopy. TMTC 3 disease variant constructs, engineered to harbor any resulting non-native residues and C-terminal S-tags, were transfected into HEK293T cells prior to cell lysate and media fractions being affinity purified with S-protein agarose beads followed by glycosidase treatment. Shifts upon PNGaseF treatment (Figure 2.8B, lanes 3 and 9) were observed for all constructs indicating that these proteins were targeted to the ER and received N-linked glycans. A 
similar increase in mobility was observed upon Endo $\mathrm{H}$ treatment indicating that the carbohydrates were high mannose glycoforms (Figure 2.8B, lanes 2 and 8). All TMTC3 disease variants co-localized with calreticulin (CRT), while co-localization was not observed with the Golgi marker GM130 when immunofluorescence staining was performed in transfected COS7 cells (Figure 2.9). Therefore, the glycosylation profiles and the cellular distribution are consistent with TMTC3 disease variants residing in the ER like WT TMTC3.

The only exception to ER localization studies was the frameshift mutation R488Efs. This mutant did not express in COS7 cells so cellular localization via immunofluorescence was unable to be determined. In order to assess expression of R488Efs S-tag, MG132, a proteasome inhibitor, was added to the media of cultured cells transfected with the S-tagged mutant construct. The immunoblot revealed that R488Efs was stabilized by proteasome inhibition suggesting that once translated, it is quickly degraded (Figure 2.10C, compare lane 3 to 4). Additionally, a glycosylation profile was obtained using proteasome inhibition (Figure 2.8B, top right panel). A shift upon EndoH and PNGaseF treatment was not observed, therefore this mutant may not be glycosylated. This could be explained by the predictive organizational outcome of this variant as the frameshift results in a severe truncation of the protein, including most of the predicted $\mathrm{N}$ linked glycosylation sites (Figure 2.8A).

The rapid degradation of the R488Efs variant suggested that instability of TMTC3 could lead to disease, therefore the stability of the remaining disease variants was analyzed. HEK293T cells were transfected with the S-tagged TMTC3 mutant constructs and cells were treated with cycloheximide for 0-24 hr. Cells were collected at the 
indicated time points, lysed and subjected to S-protein affinity purification (Figure 2.10A). WT TMTC3 has a half-life of $\sim 12 \mathrm{hr}$ (Figure $2.10 \mathrm{~B}$, black line). Several of the mutants show similar or slightly extended half-lives; however, F562Lfs and G384E mutations lead to significantly reduced half-lives of $\sim 8$ and $2 \mathrm{hr}$, respectively (Figure 2.10B, light blue and purple lines). This increased instability could explain a disease phenotype. Three of the disease variants are rapidly recognized as non-native and degraded. Further biochemical investigation is necessary to better understand the effects of such mutations.

\subsubsection{TMTC3 and TMTC4 knockdown delays gastrulation in Xenopus laevis.}

Tissue morphogenesis during development is dependent upon the cadherin family of cell-cell adhesion molecules, which includes classical cadherins, protocadherins and atypical cadherins (Halbleib and Nelson, 2006). During vertebrate morphogenesis, the various cadherin family members are differentially expressed in a variety of tissues (Nandadasa et al., 2009). E-cadherin has been shown to play a central role in cell-cell adhesion during embryo development before implantation. If O-mannosylation is prevented either genetically or biochemically, embryo development is arrested (Lommel et al., 2013). Staining for E-cadherin during mouse embryonic development showed that it is retained basolaterally, however, it was reduced at sites that also showed reduced adherence.

Analysis of the TMTCs protein sequence conservation among commonly studied species revealed strong similarity to the TMTC paralogues in Xenopus laevis, therefore the potential phenotypic effects of TMTC knockdown in developing Xenopus embryos was assessed (Figure 2.11A). In Xenopus, C-cadherin is the major cadherin expressed 
maternally persisting during early cell division and gastrulation. During gastrulation, Ecadherin is expressed in the ectoderm. At the end of gastrulation, the ectoderm becomes segregated into the dorsal neural ectoderm, which then activates expression of $\mathrm{N}$-cadherin and turns off E-cadherin. The non-neural ectoderm retains E-cadherin expression becoming the epidermis (Nandadasa et al., 2009). A global mRNA expression study conducted in developing Xenopus embryos showed that TMTC3 and TMTC4 mRNA are maternally expressed and that TMTC3 is strongly expressed during the mid-blastula transition (MBT) and remains abundant throughout gastrulation (Figure 2.11B) (Session et al., 2016). As both E-cadherin and TMTC3 and TMTC4 mRNA begin to increase in expression prior to gastrulation phase, the effect of TMTC knockdown was observed during gastrulation.

Morpholino sequences (Genetools LLC) were designed to match the ATG region of Xenopus laevis TMTC1 to 4 to block protein translation of both allo allele (L and S). Individual morpholinos were microinjected into embryos at the 1-cell stage to ensure that every cell contained morpholino upon subsequent divisions. The injected embryos were allowed to develop as normal and gastrulation was observed via time-lapse video microscopy using an inverted light microscope. The diameter of the blastopore was measured during gastrulation (stage 10-12) for all injected embryos as well as noninjected controls. Those injected with the TMTC1, 2, 3 or 4 morpholino showed a significant delay in gastrulation as exhibited by a larger blastopore diameter than noninjected controls at the same time point (Figure 2.12A and B). The most significant effect was observed for TMTC3 and TMTC4 morpholino injected embryos. With TMTC3 mRNA showing the highest expression in development (Figure 2.11B) and to confirm 
specificity of this delay in gastrulation, human WT TMTC3 mRNA was subsequently injected after the TMTC3 morpholino in 1-cell stage embryos and gastrulation was observed as previously described (Figure 2.12A and B). Human WT TMTC3 was able to completely rescue the delay in gastrulation caused by knocking down Xenopus TMTC3, suggesting that the developmental defect is specific to TMTC 3 and that the human and Xenopus proteins share similar functions.

The O-mannosyltransferases POMT1/2 and Pmt1-7 contain diacidic motifs positioned near the membrane in the lumen of the ER, which are hypothesized to serve as the active site for the transferase activity (Figure 2.4B) (Bai et al., 2019; Lommel et al., 2011). TMTC3 has two N-terminal diacidic motifs that are proposed to be luminally orientated, Asp31Asp32 (DD) and Glu73Glu74 (EE) (Figure 2.4C). To determine whether a putative active site could be assigned to TMTC 3 , the diacidic motifs were mutated to di-Ala motifs. The TMTC3 DD>AA and EE>AA constructs were injected at the 1-cell stage with TMTC3 morpholino. While injected embryos were observed for gastrulation, a number of embryos were also collected to assess construct expression. Briefly, embryos were homogenized in lysis buffer prior to total protein precipitation with trichloroacetic acid and analysis by SDS-PAGE and immunoblotting for S-tag epitope (Figure 2.11C). The analysis showed that all constructs, human WT TMTC3, DD>AA and EE>AA were similarly expressed. While the WT TMTC3 was able to rescue the gastrulation defect, neither the DD>AA nor EE>AA construct could (Figure 2.12A and B). Given that a point mutation in TMTC3 (G384E) resulted in significantly decreased stability of the protein (Figure 2.10A and B), it is possible that mutating the 
two negatively charged residues within the first luminal loop of TMTC3 to neutral Ala could affect function of TMTC3.

Mutations in TMTC3 have been identified in patients with diseases resulting from defects in cell migration. Since the process of gastrulation in metazoans is dependent upon a series of complex and coordinated cell movements, TMTC3 disease variants were assessed in the previously described Xenopus gastrulation assay (DeSimone et al., 2007; Winklbauer, 2012). One-cell stage embryos injected with TMTC3 morpholino were subsequently injected with human TMTC3 mRNA harboring three of the point mutations found in patients, H67D, R71H and G384E (Figure 2.8A). The embryos were allowed to develop to gastrulation prior to being assessed as previously described. TMTC3 morpholino injected embryos display an average increased blastopore diameter of $\sim 880$ $\mu \mathrm{m}$ compared to non-injected or WT TMTC3 rescue controls, $\sim 530$ and $\sim 600 \mu \mathrm{m}$, respectively (Figure 2.12A and B). TMTC3 disease mutants H67D, R71H and G384E possessed increased blastopore diameters of $\sim 1100, \sim 1000$ and $\sim 930 \mu \mathrm{m}$, respectively. This data shows that while WT TMTC3 rescues the gastrulation defect, the disease variants resulting in point mutations in TMTC3 increased developmental delays suggesting that they may act as dominant negative mutants.

\subsection{Discussion}

Our study demonstrates that TMTC3 O-mannosylates E-cadherin and this modification is important for E-cadherin mediated cell-cell adhesion. TMTC3 is an integral ER membrane protein, likely adopting a polytopic structure, with its putative TPR region residing within the ER lumen. The knock down of Tmtc3 in Xenopus laevis led to a delay in embryonic gastrulation, a process that is heavily dependent on cell-cell 
adhesion and migration (Winklbauer, 2012). Although the TMTCs all share similar protein domain architecture and the TMTC mRNA profiles is comparable to previously known O-mannosyltransferases, POMT1 and 2, TMTC3 appears to play a critical role in cell adhesion. This study provides insight into cell migration diseases caused by mutations in TMTC3.

TMTC3 shares $24-28 \%$ identity and $38-43 \%$ similarity in amino acid sequence with the other members of the TMTC protein family, suggestive of a common evolutionary origin (Sievers et al., 2014). TMTC3 and TMTC4 are present in the chordata phylum and recent analysis shows that they are even conserved in protists (Larsen et al., 2019). Like TMTC1 and TMTC2, TMTC3 and TMTC4 are divided into two regions that appear to create distinct domains, an N-terminal hydrophobic region and a C-terminal domain consisting of large TPR clusters (Sunryd et al., 2014). $\Delta \mathrm{G}$ predicts that TMTC3 and TMTC4 have ten and twelve potential transmembrane domains, respectively (Figure 2.1A). Alkaline extraction of total membrane preparations demonstrated that TMTC3 and TMTC4 are integral membrane proteins and trypsin digestion of these preparations produced partially protected C-terminal fragments of both TMTC3 and TMTC4 that correspond to the entire predicted C-terminal TPR region, as well as a number of the hydrophobic segments. Therefore, TMTC3 and TMTC4 appear to be polytopic membrane proteins with their hydrophobic $\mathrm{N}$-termini providing long stretches of multiple hydrophobic segments and exposed cytoplasmic loops.

Protein O-mannosylation involves the transfer of a mannose from an activated dolichol monophosphate mannose to the hydroxyl group on a Ser or Thr. The Omannosyltransferases activity for yeast PMTs appears to involve a diacidic motif (Asp- 
Glu) that resides near the membrane in luminal loop 1 of the polytopic proteins (Figure 2.4B) (Bai et al., 2019; Lommel et al., 2011). TMTC3 possesses two diacidic motifs in the first predicted luminal loop (Asp31Asp32 (DD) and Glu73Glu74 (EE)) (Figure 2.4C). These diacidic residues were mutated to Ala and the stability of TMTC3 was monitored (Figure 2.13B, lower panel). While TMTC3EE>AA was expressed at similar levels to WT TMTC3, TMTC3DD>AA was not visualized after transfection. To determine if TMTC3DD>AA was unstable, a cycloheximide chase was carried out for WT TMTC3, TMTC3DD>AA, and TMTC3EE>AA (Figure 2.13C). TMTC3DD>AA was turned over rapidly when compared to WT TMTC3 and TMTC3EE>AA indicating that TMTC3DD>AA was unstable. Even though the instability of TMTC3DD>AA prohibited us from comparing its ability to rescue the O-mannosylation of E-cadherin in COSMC/POMGnT1/TMTC1/2/3/4--- HEK293 cells to WT TMTC3 and TMTC3EE>AA, we were able to show that TMTC3EE>AA rescues O-glycosylation of E-cadherin as Ecadherin still binds Con A in TMTC3EE>AA transfected cells (Figure 2.13B, upper panel lane 8). This potentially links the TMTC3 Asp31Asp32 (DD) diacidic motif to its activity. In Xenopus, neither the TMTC3EE>AA nor TMTC3DD>AA construct was able to rescue the delay in gastrulation caused by the TMTC3 morpholino (Figure 2.12A and B). These results indicated that the diacidic residues in loop 1 were important for protein stability and possibly the transferase activity.

The glycosylation profiles combined with the trypsin protection of the C-terminal domains, placed the TPRs within the ER lumen for both TMTC3 and TMTC4. This is similar to the TPR regions of TMTC1 and TMTC2 (Sunryd et al., 2014). TMTC3 and TMTC4 encode eleven and eight TPR motifs, respectively, according to TPRPred 
(Karpenahalli et al., 2007). TPR domains are found in proteins across species and organelles and participate in a number of activities including protein translocation, folding, and post-translational modification (Graham et al., 2019). Furthermore, TPRcontaining proteins comprise the largest functional family within the cellular proteostasis/chaperome network (Brehme et al., 2014). TPRPred predicted that the eleven TPR motifs of TMTC3 are organized into clusters of three and eight, while TMTC4 has eight sequential TPRs. The differences in the organization of the TPR domains for TMTC 3 and TMTC4 could have implications for their functions.

Extensive bioinformatic analysis shows that long stretches of consecutive TPR domains (observed up to sixteen) tend to be more promiscuous in their selectivity (Magliery and Regan, 2005). Given that TMTC3 and TMTC4 both possess a stretch of eight consecutive motifs, this suggests that they may bind a number of substrates. This is most similar to the well-studied TPR-containing cytoplasmic O-GlcNAc transferase (OGT), which contains a stretch of 12.5 TPRs. The structure of OGT revealed that the TPRs form a super helix with conserved Asn along the concave surface that form a continuous ladder (Figure 1.1C). The Asn in OGT that are particularly well-conserved are those at positions 6 and 9 in the TPR consensus and thought to contribute to inter-repeat interactions (Jínek et al., 2004). Seven of the eleven TPRs in TMTC3, as predicted by TPRPred, have Asn at position six and seven out of eight of the TPRs in TMTC4 have Asn at position six in addition to two of the eight having Asn at position nine. The number of conserved Asn at positions six and nine of the predicted TPRs in TMTC 3 and TMTC4 suggests that they may form super helical structures and use the Asn to nucleate protein-protein interactions as this also mimics the Asn conservation observed in the 
ARM-repeat proteins importin- $\alpha$ and $\beta$-catenin, which contribute to the binding of the target peptide (Conti et al., 1998; Huber and Weis, 2001). $\beta$-catenin was shown to interact with E-cadherin through conserved Asn on its ARM-repeat domains (Huber and Weis, 2001). $\beta$-catenin has twelve ARM-repeat domains and five of the domains contain an Asn at position 13 and are responsible for recognizing the backbone of extended peptides. Furthermore, alignment of murine cadherin family sequences (M-cadherin, OBcadherin, desmocollin 1a and desmoglein 1) to E-cadherin show that many of the observed E-cadherin $/ \beta$-catenin interactions are likely to be conserved among other cadherin family members. TMTC 3 contains seven Asn at a conserved position on its TPR motifs, which could be used to interact with the well conserved family of cadherins in order to recruit the substrates for O-mannosylation near the ER membrane (Figure 4.1B). Analysis of TPR-containing protein structures and their ligands revealed that smaller clusters of three and four TPRs corresponds to co-chaperone function (Brehme et al., 2014; Graham et al., 2019). Like one of the most well-studied TPR structures in the protein HOP, the N-terminal cluster of three TPRs in TMTC3 may bind and recruit chaperones for protein homeostasis, analogous to the MIR domains in the POMT/Pmts (Fujimori et al., 2017; Scheufler et al., 2000). MIR domains are observed in six nonredundant proteins, all of which target to the ER (Letunic and Bork, 2018; Nielsen, 2017). Stromal cell-derived factor 2 (SDF2) is a small protein of 211 amino acids comprised primarily of three MIR domains. While not much is known about MIR domain function for SDF2 and its isoform, SDF2L1, their MIR domains have been found to regulate the chaperone cycle of BiP (Fujimori et al., 2017). Both proteins form a stable complex with the ER J-domain protein ERdj3 and inhibit the aggregation of misfolded 
ER cargo by binding non-native proteins and promoting the BiP-substrate interaction cycle. We hypothesize that the three TPR motif cluster of TMTC3 may form a similar complex with chaperones for the delivery of unfolded substrate in order to expose putative sites for O-mannosylation.

Thirty-seven members of the cadherin superfamily of cell membrane receptors were identified as major carriers of O-mannose glycans (Vester-Christensen et al., 2013). Cell-surface receptors of the cadherin family mediate cell-cell adhesion at sites of cellcell contact, which is crucial for cells to function in an integrated manner (Lodish et al., 2000; Meng and Takeichi, 2009). In our study, cells lacking TMTC1-4 stained for cadherins display cell-cell attachment irregularity appearing less round and consistent in shape (Figure 2.7A). In combination with the data demonstrating that TMTC3 is involved in the O-mannylation of E-cadherin, this suggests that TMTC3 plays a role in cellular adherence through the O-mannosylation of E-cadherin.

Cadherins contain multiple repeats of extracellular cadherin (EC) domains and they mediate cell-cell adhesion by trans homodimerization between the most distal EC1 and EC1-2 domains on apposed cells (Brasch et al., 2012; Cavallaro and Christofori, 2004; Yagi, 2008). The other EC domains play a critical role in presenting the EC1 and 2 domains so that they may form these homodimers. The O-mannose modified sites identified are confined to the EC2-5 domains of both classic type 1 and 2 cadherins and these sites also appear to be evolutionarily conserved. E-cadherin, a type I classical cadherin, holds most epithelial sheets together and is highly abundant at the sites of cellcell contact along lateral surfaces (Lodish et al., 2000). We found that COSMC/POMGnT1/TMTC1/2/3/4-/- HEK293 cells adhere less to the immobilized 
extracellular region of E-cadherin than HEK293 COSMC/POMGnT1-/ and COSMC/POMGnT1/POMT1/2-- cells suggesting that genetic manipulation of the $T M T C$ s affects E-cadherin's ability to transhomodimerize (Figure 2.7D and E). Complementation with TMTC3 in COSMC/POMGnT1/TMTC1/2/3/4-- HEK293 cells increased E-cadherin mediated cell-cell adhesion, therefore demonstrating that TMTC3 O-mannosylation of E-cadherin plays a crucial role in its ability to homodimerize (Figure 2.7D and E, and Figure 2.5). Given that E-cadherin is also crucial during embryogenesis, the effects we observe upon knockdown of Tmtc3 and subsequent rescue of the gastrulation delay with human TMTC3 WT, could be attributed to the role TMTC3 plays in E-cadherin mediated cell-cell adhesion and O-mannosylation (Figure 2.12A and B) (Halbleib and Nelson, 2006).

Mutations in TMTC3 were identified in patients with Cobblestone lissencephaly and periventricular nodular heterotopia (PVNH), common brain malformations caused by defects in neuronal migration (Farhan et al., 2017; Jerber et al., 2016). More specifically, three of the mutations (R488Efs, F562Lfs and G384E variants) identified in Cobblestone lissencephaly patients that possess a significant number of clinically observed neurological defects were found to be unstable in our protein degradation assay (Figure 2.10) (Jerber et al., 2016). The rapid degradation of these variants could explain the development delays observed in patients due to lack of TMTC3. Our Xenopus developmental studies revealed that two of the mutants (H67D and R71H) lead to a developmental delay even though they were more stable in cells. Our characterization of the disease variants of TMTC 3 provides a link between a previously uncharacterized protein and disease resulting from its mutation. 
TMTC1-4 are not only implicated in the O-mannosylation of cadherins but were also found to be involved in the O-mannosylation of several ER/Golgi resident proteins, including ERp57, ERdj4 and FKBP10 (Larsen et al., 2017a). Each of these are important chaperones for protein folding of the secretory pathway aiding in disulfide bond formation, $\mathrm{BiP}$ activation and proline cis trans isomerization. O-mannosylation of both trafficked and resident secretory pathway proteins could contribute to their quality control either through increasing favorable folding or enhancing degradation of specific substrates upon misfolding. While previous studies show that $\mathrm{O}$-mannosylation is an important structural modification, recent studies show that it may also play a role in ER quality control (ERQC) (Xu and $\mathrm{Ng}, 2015)$. If certain mutants are stabilized, they could cause dysregulation of protein homeostasis due to an excess of O-mannose modification of particular ER resident chaperones.

In conclusion, we demonstrate that TMTC3 is crucial for O-glycosylation of Ecadherin and cell-cell adhesion and embryonic development. Evidence shows that although the TMTCs share architectural similarity, they perform distinct functions and help to regulate cellular homeostasis by participating in essential processes, posttranslational modification and calcium regulation. TMTC3 directed O-mannosylation is biologically important and further studies are of interest to better understand TMTC3's function within the cell and how mutations in this gene cause disease.

\subsection{Materials and Experimental Procedures}

\subsubsection{Plasmids and reagents}

Dulbecco's modified Eagle's medium (DMEM), DMEM GlutaMax, fetal bovine serum, penicillin and streptomycin were purchased from Invitrogen/ThermoFisher 
Scientific. Easy-Tag [35S]-Cys/Met was purchased from PerkinElmer Life Sciences. Sprotein-agarose beads and S-tag antibody were purchased from EMD Millipore. Endo H, PNGase F, Protoscript II first strand cDNA synthesis kit and all cloning reagents were purchased from New England Biolabs. FastStart SYBR Green qPCR mix was purchased from Roche Diagnostics, and all primers were acquired from IDT DNA. IRDye® 800CW Goat anti-Mouse IgG and IRDye ${ }^{\circledR}$ 680RD Goat anti-Rabbit IgG were purchased from LiCOR Biosciences. Protein A sepharose CL-4B was purchased from GE Healthcare. Antibodies directed toward the following antigens were also purchased: calnexin (Enzo Life Sciences); calreticulin (ThermoFisher Scientific); E-cadherin (GeneTex); pancadherin (Sigma); ERp57 (gift from Dr. Taku Tamura, Akita University, Japan), GM130 (BD Biosciences) and KDEL (Enzo Life Sciences). TMTC3 and TMTC4 cDNA was created by isolating RNA (HEK293T cells) and cloned into pcDNA3.1 A-, a plasmid harboring a C-terminal S-tag, using standard molecular biology techniques. All other chemicals were obtained from Sigma.

\subsubsection{Cell lines/Tissue Culture}

HEK293T, 293A or COS7 cells were grown in DMEM supplemented with 10\% fetal bovine serum, $100 \mathrm{U} / \mathrm{mL}$ penicillin and $100 \mathrm{mg} / \mathrm{ml}$ streptomycin. HEK293 cell lines (COSMC/POMGNT1--, COSMC/POMGNT1/POMT1/2--, and COSMC/POMGNT1/TMTC1/2/3/4---) were generously contributed by Dr. Henrik Clausen (University of Copenhagen) and grown in DMEM GlutaMax supplemented with $10 \%$ fetal bovine serum.

\subsubsection{In silico analysis of TMTC3 and TMTC4}


The primary amino acid sequences of TMTC 3 and TMTC4 were analyzed by UniProtKB and TPRpred to identify the number and position of putative TPR domains (Xu and Ng, 2015)(Karpenahalli et al., 2007, 2019). Hydrophobic domains were identified by the $\Delta \mathrm{G}$ software, which predicts transmembrane domains (Hessa et al., 2007). Putative N-linked glycosylation sites were identified by NetNGlyc (Gupta, 2002).

\subsubsection{Affinity Purification and glycosylation assay}

Transfected cells were lysed in MNT buffer (0.5\% Triton X-100, 20 mM MES, $100 \mathrm{mM} \mathrm{NaCl}, 30 \mathrm{mM}$ Tris- $\mathrm{HCl}[\mathrm{pH} 7.5])$. All steps were conducted at $4{ }^{\circ} \mathrm{C}$. The postnuclear supernatant (PNS) was isolated by centrifugation followed by pre-clearing with un-conjugated agarose beads for $1 \mathrm{hr}$. Cleared supernatant was incubated with S-protein agarose beads overnight and subsequently washed twice with wash buffer $(0.05 \%$ Triton$\mathrm{X}-100,0.1 \% \mathrm{SDS}, 300 \mathrm{mM} \mathrm{NaCl}, 100 \mathrm{mM}$ Tris- $\mathrm{HCl}$ [pH 8.6]). After the final wash, glycosylation assays were performed by adding appropriate buffers and either mock, Endo H or PNGase F enzymes according to the manufacturer's protocol. Finally, reducing sample buffer was added to all samples and they were analyzed by SDS-PAGE.

\subsubsection{Confocal Microscopy}

Cells were fixed with 3.7\% paraformaldehyde in phosphate buffered saline (PBS) for $15 \mathrm{~min}$ followed by permeabilization with $0.1 \%$ triton $\mathrm{X}-100$ for $15 \mathrm{~min}$ at $25^{\circ} \mathrm{C}$. Slides were stained with the indicated primary antibodies followed by staining with appropriate Alexa Fluor 488 or 594 secondary antibodies in immunostaining buffer (10\% fetal bovine serum in $1 \mathrm{X}$ PBS). Slides were rinsed and mounted onto cover slips with VectaShield (Vector Laboratories). Images were obtained with a Fluoview 1000 MPE, 1X81 motorized inverted research microscope (Olympus Inc.) equipped with a 
Hamamatsu C8484-05G camera. All images were acquired with a Plan Apo N 60x 1.42NA lens and processed by using the FV10-ASW and Adobe Photoshop software. For pan-cadherin staining, 24-well glass bottomed plates were prepared with $0.1 \%$ gelatin in PBS for $1 \mathrm{hr}$ at $37^{\circ} \mathrm{C}$ prior to coating each well with $10 \mu \mathrm{g} / \mathrm{mL}$ fibronectin (Sigma) overnight at $4{ }^{\circ} \mathrm{C}$. Cells were allowed to adhere in the wells for $2 \mathrm{hr}$ at $37^{\circ} \mathrm{C}$ and subsequently fixed in methanol for $10 \mathrm{~min}$ at $-20{ }^{\circ} \mathrm{C}$. Cells were stained with the indicated primary antibodies followed by staining with appropriate Alexa Fluor 488 or 594 secondary antibodies in $1 \mathrm{X}$ PBS containing $0.1 \%$ Tween, $2 \%$ bovine serum albumin (Fisher) and $0.02 \%$ sodium azide. Cells were rinsed and then incubated with Hoescht (Abcam) for $1 \mathrm{hr}$ at $25^{\circ} \mathrm{C}$. Images were obtained with a A1R: Nikon A1 resonant scanning confocal with TIRF module microscope equipped with an Andor Xyla camera. All images were acquired with a Plan Apo IR 60x 1.27WI lens and processed by using the Nikon Elements and Adobe Photoshop software.

\subsubsection{Alkaline extraction}

Alkaline extraction was performed as previously described (Sunryd et al., 2014). Briefly, radiolabeled cells were resuspended in ice-cold homogenization buffer $(20 \mathrm{mM}$ HEPES, $5 \mathrm{mM} \mathrm{KCl,} 120 \mathrm{mM} \mathrm{NaCl}, 1 \mathrm{mM}$ EDTA, and $0.3 \mathrm{M}$ sucrose [pH 7.5]) and passed through a 25 -gauge needle 20 -times. All subsequent steps were conducted at $4{ }^{\circ} \mathrm{C}$. The homogenate was centrifuged at $1,000 \mathrm{~g}$ for $10 \mathrm{~min}$ to pellet the nuclear fraction. The remaining PNS was centrifuged at 45,000 rpm in Beckman rotor (TLA 120.2) for 10 min to separate the cytosol (supernatant) from the cellular membranes (pellet). The cellular membrane fraction was resuspended in homogenization buffer, and a portion of the 
resuspended membranes was incubated with $0.1 \mathrm{M} \mathrm{Na}_{2} \mathrm{CO}_{3}(\mathrm{pH} 11.5)$ for 30 min on ice. The alkaline extracted portion was centrifuged at $65,000 \mathrm{rpm}$ for $20 \mathrm{~min}$ through a sucrose cushion (50 mM triethanolamine, $0.3 \mathrm{M}$ sucrose [pH 7.5]) to separate soluble proteins from membrane proteins in the supernatant and pellet, respectively. The $\mathrm{pH}$ was adjusted in the alkaline extracted sample with $1 \mathrm{M}$ Tris- $\mathrm{HCl}(\mathrm{pH}$ 7.5). An excess of MNT was added to all fractions, and immunoprecipitation or affinity precipitation was performed with protein-A sepharose and appropriate antisera or with S-protein agarose, respectively.

\subsubsection{Trypsin protection}

Transfected cells were resuspended in cold homogenization buffer $(10 \mathrm{mM}$ HEPES [pH 7.4], $10 \mathrm{mM} \mathrm{KCl,} 1.5 \mathrm{mM} \mathrm{MgCl,} 5 \mathrm{mM}$ sodium EDTA, 5 mM sodium EGTA and 0.25 M sucrose) and passed through a 25-gauge needle 20-times. All subsequent steps were conducted at $4{ }^{\circ} \mathrm{C}$. The homogenate was centrifuged at $1,000 \mathrm{~g}$ for $10 \mathrm{~min}$ to pellet the nuclear fraction. The remaining PNS was centrifuged at 45,000 rpm in Beckman rotor (TLA 120.2) for 10 min to separate the cytosol (supernatant) from the cellular membranes (microsomes). The microsomes were resuspended in homogenization buffer containing $0.1 \mathrm{M} \mathrm{NaCl}$ and $10 \mu \mathrm{g}$ trypsin and/or triton $\mathrm{X}-100$ was added to a final concentration of $0.1 \%$. After incubation at $27^{\circ} \mathrm{C}$ for $15 \mathrm{~min}$, the reaction was quenched with $100 \mu \mathrm{g}$ soybean trypsin inhibitor. Reducing sample buffer was added and analyzed via SDS-PAGE.

\subsubsection{Immunoblotting, endoglycosidase and affinity purification}

Non-transfected and transfected cells were lysed in MNT and the post-nuclear supernatant (PNS) was isolated by centrifugation followed by total protein concentration 
determination using 595 nm Protein Assay Dye Reagent (Bio-Rad). All subsequent steps were conducted at $4{ }^{\circ} \mathrm{C}$. Equal amounts of total protein were split into three fractions. Preclearing was performed on one of the fractions with control agarose beads for $1 \mathrm{hr}$ prior to affinity purification with S-protein agarose beads overnight. The remaining two fractions were subjected to a glycosylation assay by adding appropriate buffers and either mock or PNGase F enzyme according to the manufacturer's protocol. Following endoglycosidase treatment, affinity purification for glycoproteins using Con A (Sigma) was performed overnight. Beads were washed twice in wash buffer. Finally, reducing sample buffer was added to all samples and they were analyzed by SDS-PAGE. Proteins were transferred to a polyvinylidene difluoride membrane and immunoblotted with the appropriate antisera. Blots were developed and TIFF files were acquired using a LI-COR Odyssey CLx Imager. Densitometric quantification of western blots was performed using ImageJ software (Fiji). The amount of E-cadherin bound to Con A was calculated by dividing the amount of E-cadherin in the PNGaseF treated transfected cells with the amount of E-cadherin in the PNGaseF treated non-transfected cells (mock). The amount of E-cadherin in non-transfected cells was set to $100 \%$. Error bars represent the standard deviation for three independent experiments. TMTC3 S-tag transfected samples were also subjected to a glycosylation and carbohydrate binding assay that included both PNGaseF and $\alpha 1-2,3,6$ mannosidase treatment. Briefly, transfected cells were lysed as described above and split into three fractions. Glycosylation assays were performed by adding appropriate buffers and either mock or PNGase F enzymes according to the manufacturer's protocol and subsequent treatment with $\alpha 1-2,3,6$ mannosidase enzyme and appropriate buffers for $24 \mathrm{hr}$. Samples were analyzed as described above and the 
amount of E-cadherin bound to Con A was calculated by dividing the amount of Ecadherin in PNGaseF/ 11-2,3,6-mannosidase treated samples by the amount of PNGaseF treated E-cadherin, which was set to $100 \%$. Error bars represent the standard deviation for three independent experiments.

\subsection{9 qRT-PCR}

HEK293A cells were treated with regular growth media or dithiothreitol $(2 \mathrm{mM})$ for $2 \mathrm{hr}$ or tunicamycin $(1 \mu \mathrm{g} / \mathrm{mL})$, thapsigargin $(3 \mu \mathrm{M})$, brefeldin $\mathrm{A}(2.5 \mu \mathrm{g} / \mathrm{mL})$ and MG132 $(2.5 \mu \mathrm{M})$ for $24 \mathrm{hr}$ prior to RNA isolation with RNAeasy Mini Kit (Qiagen). One $\mu \mathrm{g}$ of purified RNA was reverse transcribed into cDNA using the Protoscript II Reverse Transcriptase kit (New England Biolabs). Quantitative real time polymerase chain reactions (qRT-PCR) were performed in $20 \mu \mathrm{L}$ reactions using the FastStart universal SYBR Green master (Rox) kit (Roche diagnostics Corp.) on an Mx3000P real-time PCR machine (Agilent Technologies Inc.) according to manufacturer's instructions. Changes in mRNA levels were calculated using the change in cycle threshold value method with $\beta$-actin as the reference gene (Pfaffl, 2001). Statistical analysis of the data was calculated using GraphPad Prism 5.0 (GraphPad software) and significance between treatment groups was determined using unpaired T-tests.

The following primers were used: $\beta$-actin (5' GCACTCTTCCAGCCTTCC 3', 5' TGTCCACGTCACACTTCATG 3'), TMTC1 (5' GCTGTTTCTATTGGCCTTTCTC 3', 5' TGTCTCTTTCACCAGCATCG 3'), TMTC2 (5'

GATGTCTTTGTCTTTCACAGGC 3', 5' TGTTTCCCATCCAGTATAACCG 3') TMTC3 (5' TTTTCCTAAGCCATCCCCTG', 5' ACAAAACCACAAAAGAGGCTG 3’), TMTC4 (5' CCCTCATTAAGTCCATCAGCG 3', 5' 
ATAACGAGAAATCCCAGGCC 3'), POMT1 (5' GTGAACTACCTCCCGTTCTTC 3', 5' CACAGGGAGCAGAAGGATTT 3'), POMT2 (5'

GGCACTGGCCTATCAACTATC 3', 5' CAACAGATTCAGCCACCAAAC 3') and BiP (5' CTGCCATGGTTCTCACTAAAATG 3', 5' TTAGGCCAGCAATAGTTCCAG 3').

\subsubsection{Cycloheximide chase analysis}

HEK293T cells were transfected with S-tagged WT TMTC3 or mutant TMTC3 for $40 \mathrm{hr}$ prior to incubation with $100 \mu \mathrm{g} / \mathrm{mL}$ of cycloheximide for indicated times. Cell lysates were prepared and the expression of S-tag TMTC3 was analyzed by Western blots. Briefly, transfected cells were lysed in MNT. Protein concentration of cell lysates was determined by using the $595 \mathrm{~nm}$ Protein Assay Dye Reagent (Bio-Rad). Western blots were carried out after 9\% SDS-PAGE. After incubation with IRDye-conjugated secondary antibodies, the protein-antibody complexes were visualized by the LI-COR Odyssey CLx Imager and densitometric quantification of western blots was performed using ImageJ software (Fiji). The stability of TMTC3 was calculated by dividing the amount of TMTC3 WT or mutants in cyclohexmide treated samples (2-24 hr time points) by the amount of TMTC3 in untreated samples ( 0 time point), which was set to $100 \%$. Error bars represent the standard error for three independent experiments.

\subsubsection{Cell Adhesion Assays}

Substrates included bovine fibronectin (Sigma), E-cadherin (R\&D Systems), and BSA (Fisher). Falcon Probind 96-well plates were coated with 25 and $50 \mu \mathrm{g} / \mathrm{ml}$ substrates in PBS overnight at $4{ }^{\circ} \mathrm{C}$, rinsed with PBS, and blocked with $10 \mathrm{mg} / \mathrm{ml} \mathrm{BSA}$ in PBS for 2 $\mathrm{hr}$ at $25^{\circ} \mathrm{C}$. Cells were harvested in serum free medium by resuspension. 
60,000 cells/well were seeded and the adhesion assay was performed for $1 \mathrm{hr}$ at $37^{\circ} \mathrm{C}$ in $5 \% \mathrm{CO}_{2}$ in a humidified atmosphere. Non-adherent cells were washed away with serum free media. Adherent cells were fixed for 20 min with $4 \%$ formaldehyde in PBS, rinsed with PBS, and permeabilized with $0.1 \%$ triton $\mathrm{X}-100$ for 20 min prior to staining with Hoescht (Abcam). Excess stain was removed with PBS. Each assay point was derived from three independent experiments.

\subsubsection{Xenopus embryo handling and media}

Eggs were obtained from adult Xenopus laevis, fertilized, and cultured as described previously (Cousin et al., 2008). Embryos were staged according to (Nieuwkoop and Faber 1994). Briefly, after in vitro fertilization, the eggs were dejellied in $2 \%$ cysteine ( $\mathrm{pH} \sim 8.3$ ) and then cultured in 3\% Ficoll in Modified Barth's Solution (1X MBS) at $15{ }^{\circ} \mathrm{C}$ until they reached the desired stage for injection. Embryos were grown in $0.1 \mathrm{X}$ MBS at either 15 or $18^{\circ} \mathrm{C}$. Time-lapse video microscopy was performed at $18^{\circ} \mathrm{C}$.

\subsubsection{Microinjection experiments}

Morpholinos (GeneTools, LLC) for TMTC1, 2, 3 and 4 were designed to block translation of the respective proteins and contain a 3'-fluorescein modification by the manufacturer and injected (10 ng) at the one-cell stage. mRNAs of human TMTC3 WT and previously described mutants, cloned into pcDNA3.1 A- with a C-terminal S-tag, were transcribed using T7 polymerase following linearization of plasmids with EagI or DraIII. Transcripts were desalted on G-50 Nick columns (Pharmacia), extracted with phenol/chloroform, and ethanol precipitated. Transcripts were quantified by absorbance 
at $260 \mathrm{~nm}$ and resuspended at $0.2 \mathrm{mg} / \mathrm{ml}$ in DEPC-treated $\mathrm{H}_{2} \mathrm{O}$. Transcripts $(0.4-1 \mathrm{ng}$ total) were injected at the one-cell stage, following injection of the TMTC3 morpholino.

\subsubsection{Embryo and cell imaging}

Pictures of embryos were taken using the Zeiss Axiovert $200 \mathrm{M}$ inverted microscope equipped with a Ludl xyz-stage control and a Hamamatsu Orca camera (Figure 2.12) or BZ-X Analyzer equipped with Nikon 10X CFI PlanFluor lens and BZX710 camera (Keyence). Images were taken using either the AxioVision software (Zeiss) or the BZ-X capture software (Figure 2.12) (BZ-X700 Analyzer). The diameter of the blastopore was measured using ImageJ software (Fiji) and quantified using GraphPad Prism 5.0 (GraphPad software). Statistical analysis between morpholino and TMTC3 construct injected groups was performed using one-way ANOVA and *, **, *** indicates a P-value of less than $0.05,0.01$ and 0.001 , respectively. Error bars represent standard deviation.

\subsubsection{Protein extraction and analysis}

Embryonic proteins were extracted from 10-20 frozen embryos using 1X MBS (Laskey et al., 1977) containing 1\% triton X-100, protease inhibitor cocktail (Sigma), 2 $\mathrm{mM}$ phenylmethylsulfonide fluoride (PMSF), and $1 \mathrm{mM}$ EDTA on ice followed by $16,000 \mathrm{~g}$ centrifugation for $30 \mathrm{~min}$ at $4{ }^{\circ} \mathrm{C}$. Supernatant was either directly added to an equal volume of $2 \mathrm{X}$ Laemmli buffer containing $2 \% \beta$-mercaptoethanol or precipitated with trichloroacetic acid (Sigma) and washed with acetone prior to resuspension in $2 \mathrm{X}$ Laemmli containing $2 \%$ of $\beta$-mercaptoethanol. Western blots were performed using standard techniques and the presence of translated TMTC3 constructs was assessed using anti-S-tag antisera. 
A
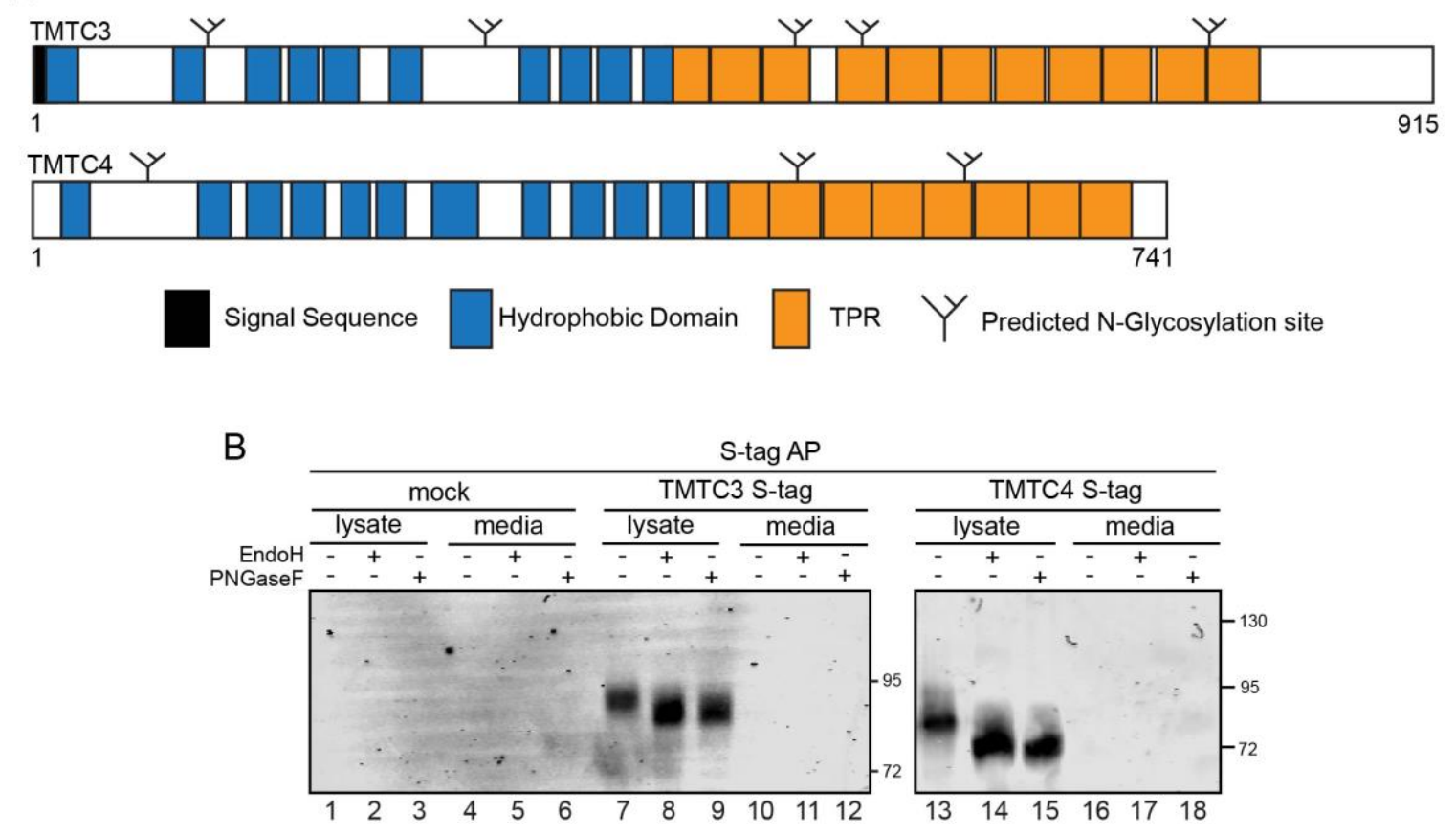

C

TMTC3
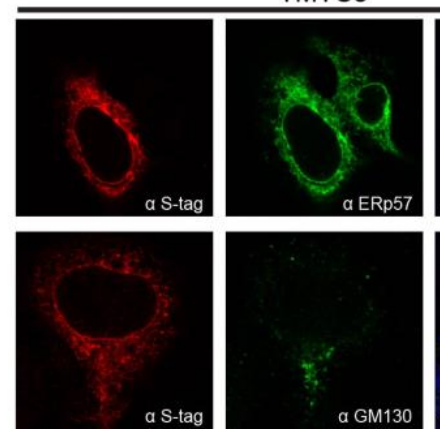
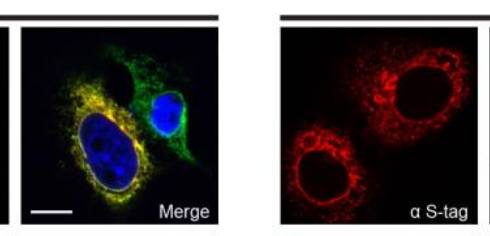

TMTC4
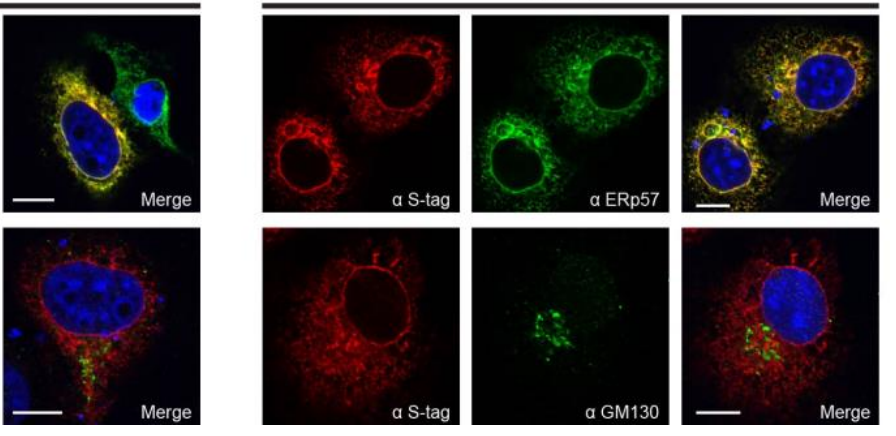

Figure 2.1. TMTC3 and TMTC4 are ER resident proteins. (A) The organization of TMTC 3 and TMTC4 with signal sequences (black), hydrophobic domains (blue) and TPR domains (orange) as designated. Predicted endogenous N-linked glycosylation sites are indicated by small black, branched structures. (B) HEK293T cells were transfected with S-tagged TMTC3 or TMTC4 as indicated and were affinity purified from the cell lysate and media using S-protein agarose. Samples were then subjected to a glycosylation assay with either EndoH (lanes 2, 5, 8, 11, 14 and 17) or PNGaseF (lanes 3, 6, 9, 12, 15 and 18) digestion as indicated. Reducing sample buffer was added and the samples were analyzed by a 6\% (TMTC3 S-tag) and 8\% (TMTC4 S-tag) SDS-PAGE. (C) Cellular localization of TMTC 3 and TMTC4 was investigated by confocal microscopy. COS7 cells were transfected with TMTC3 or TMTC4 cDNA. Fixed cells were stained with Stag, ERp57 (ER) or GM130 (Golgi) antisera. Nuclei were visualized by DAPI staining (blue). Scale bars correspond to $10 \mu \mathrm{m}$. 

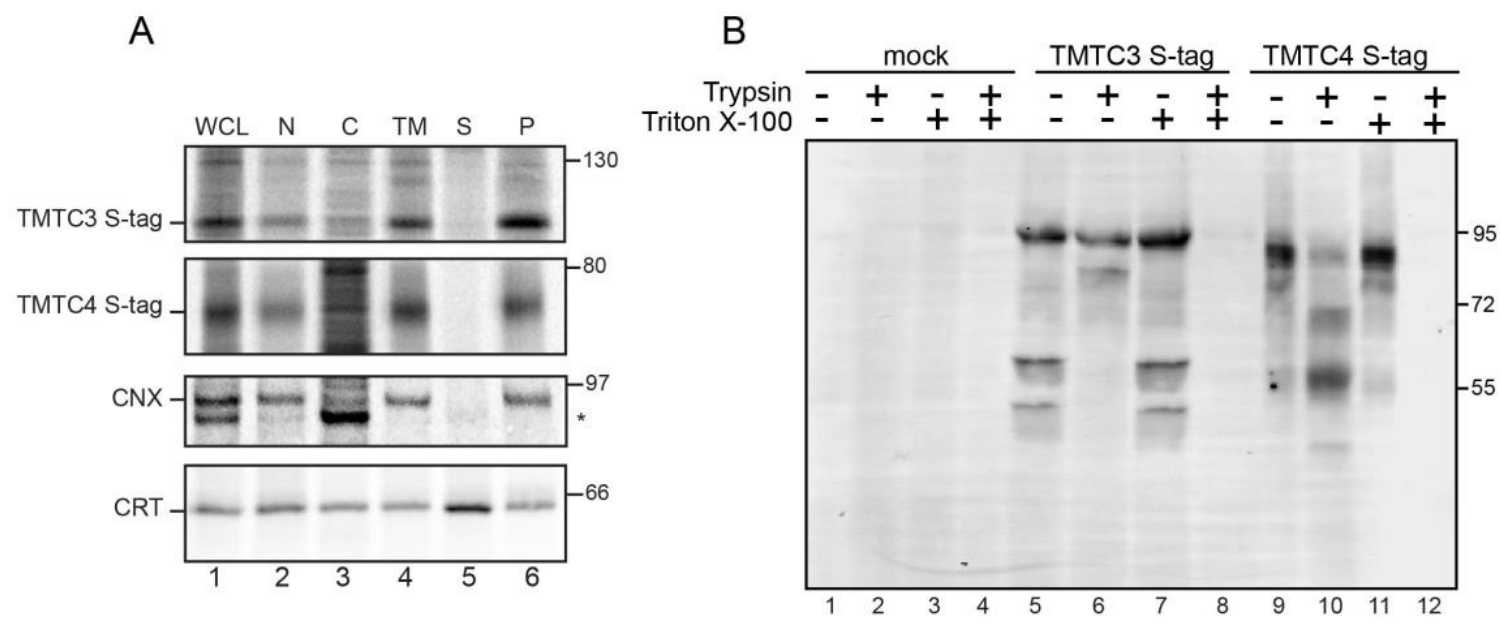

C
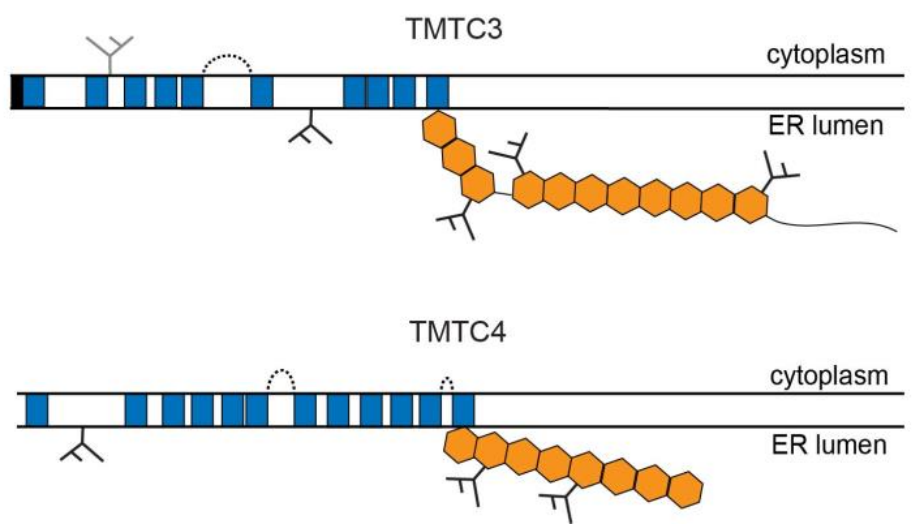

Figure 2.2. TMTC3 and TMTC4 are transmembrane proteins with their TPR domains facing the ER lumen. (A) HEK293T cells were transfected with S-tagged TMTC3 or TMTC4 as indicated and radiolabeled for $1 \mathrm{hr}$ with [35S]-Cys/Met. Cells were homogenized and fractionated prior to alkaline extraction. The fractions collected were whole cell lysate (WCL), nucleus (N), cytosol (C), total membrane (TM), as well as supernatant $(\mathrm{S})$ and pellet $(\mathrm{P})$ fractions upon alkaline extraction of the TM. All samples were subjected to affinity purification with S-tag agarose or calnexin (CNX) and calreticulin (CRT) immunoprecipitation where indicated. Samples were analyzed via SDS-PAGE and detected via autoradiography. (B) TMTC3 S-tag and TMTC4 S-tag were expressed in HEK293T cells. Cells were homogenized and microsomes were isolated by ultracentrifugation then resuspended in homogenization buffer. Aliquots of the ER microsomes were incubated for $15 \mathrm{~min}$ at $27{ }^{\circ} \mathrm{C}$ with or without triton X-100 and trypsin where indicated. Samples were separated on 9\% SDS-PAGE and immunoblotted for the S-tag epitope. (C) Putative trypsin protease cleavage areas (dashed line) on TMTC3 and TMTC4 based on exposed potential cleavage residues and size of cleaved bands in B. 
A

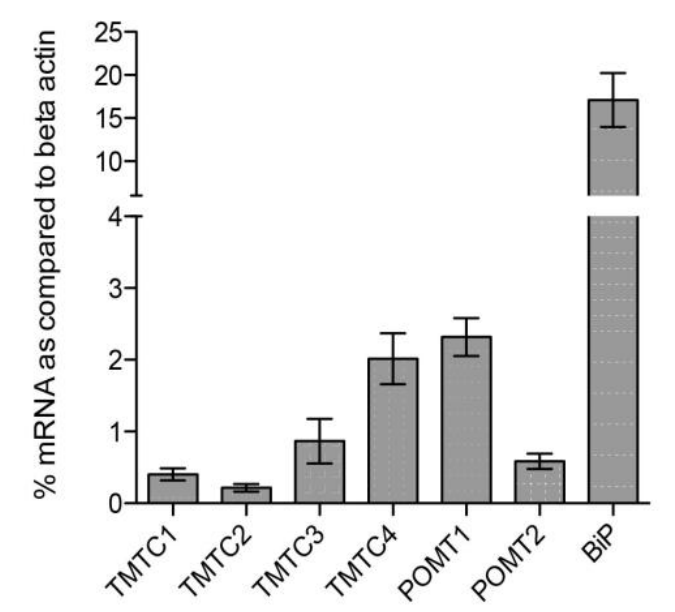

B

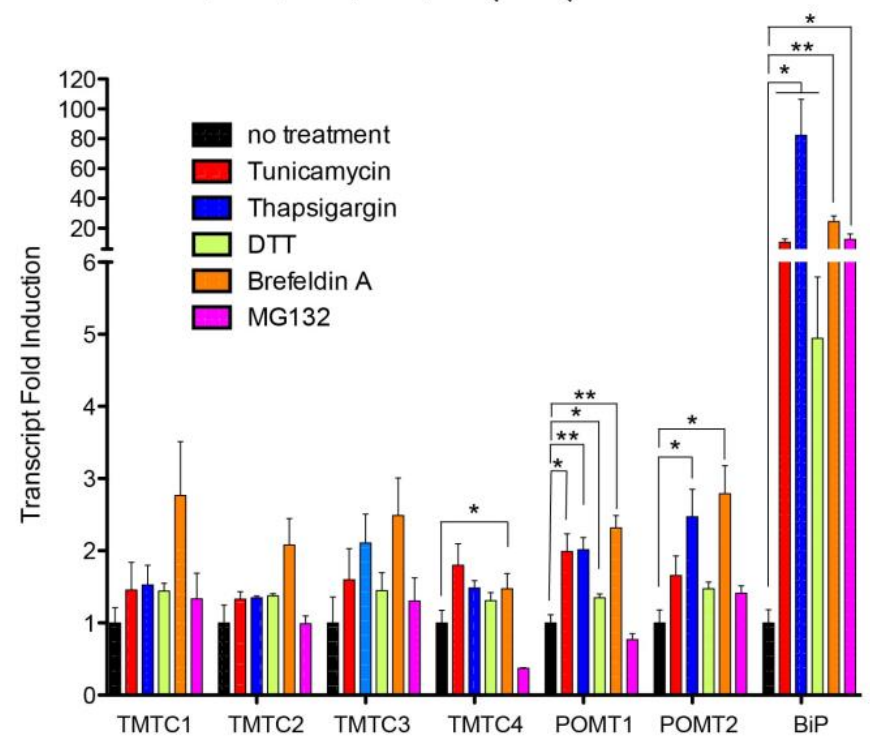

Figure 2.3. Basal transcript abundance and fold induction of TMTC proteins by ER stress. (A) RNA from HEK293A cells, grown under normal conditions, was harvested. RNA was reverse transcribed to cDNA followed by qRT-PCR with appropriate primers. Basal mRNA abundance was assessed using $\beta$-actin as a reference. Error bars represent standard error from at least three independent experiments. (B) HEK293A cells were treated with regular growth media or with $2 \mathrm{mM}$ DTT for $2 \mathrm{hr}, 1 \mu \mathrm{g} / \mathrm{mL}$ tunicamycin, 3 $\mu \mathrm{M}$ thapsigargin, $2.5 \mu \mathrm{g} / \mathrm{mL}$ brefeldin A or $2.5 \mu \mathrm{M}$ MG132 for $24 \mathrm{hr}$ prior to RNA purification. RNA was reverse transcribed to cDNA followed by qRT-PCR with appropriate primers, and changes in gene expression were calculated using $\beta$-actin as a reference. Statistical significance between treatment groups was determined using an unpaired $\mathrm{T}$ test. * and ** indicates a P-value of less than 0.05 and 0.01 , respectively. Error bars represent standard deviation from at least three independent experiments. 
A

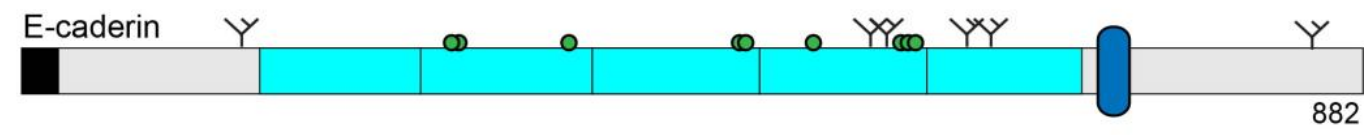

B
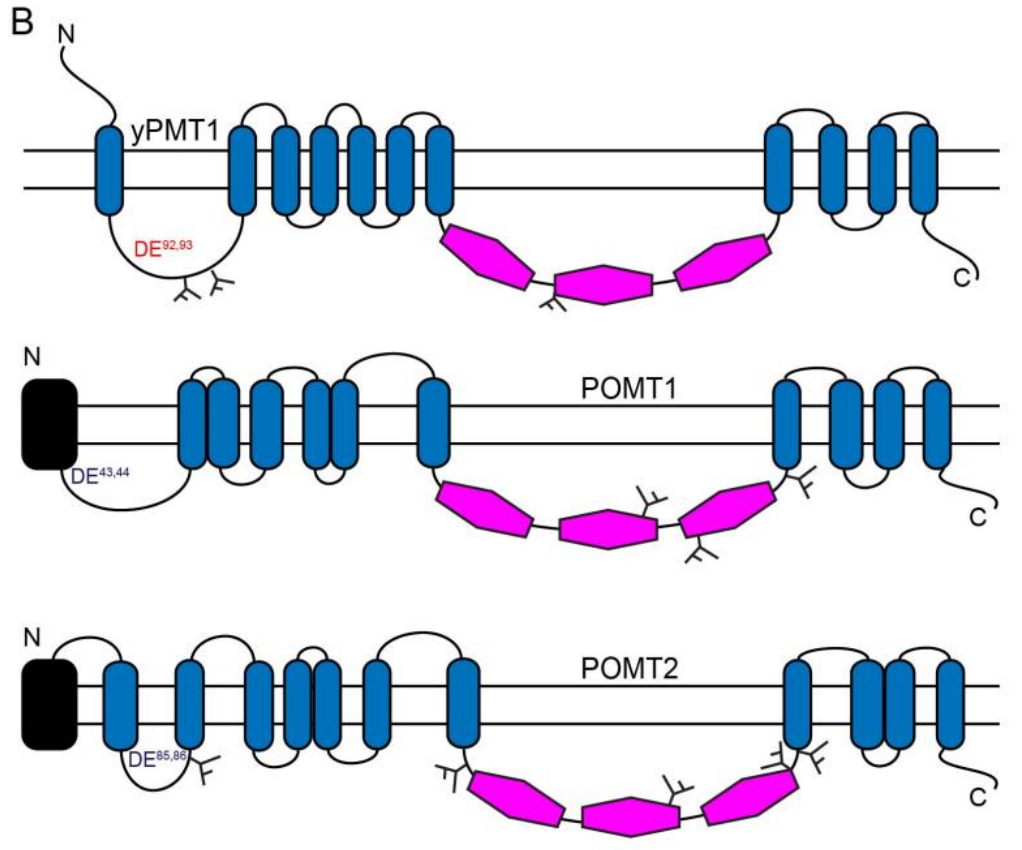

C

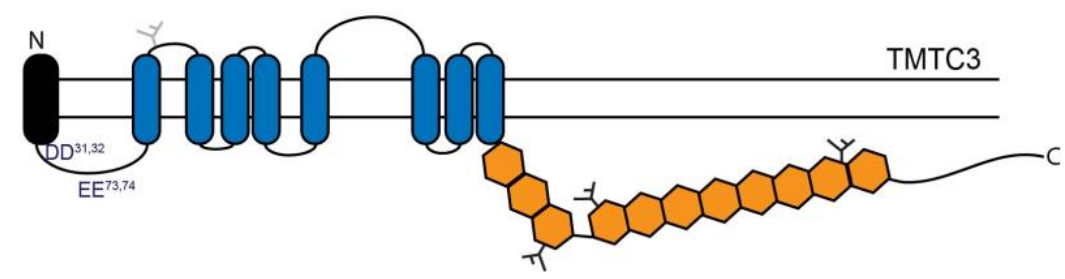

Figure 2.4. TMTCs and POMTs share similar functional domain architecture. (A) The organization of E-cadherin with signal sequence (black), extracellular cadherin domains (light blue) and transmembrane domain (dark blue) as designated. N-linked glycosylation sites are indicated by small black, branched structures and $\mathrm{O}$-mannosylation sites by green circles. (B) The organization of yeast Pmt1 and predicted organization of human POMT1 and 2 with signal sequences (black), hydrophobic domains (dark blue) and MIR domains (pink) as designated. Predicted endogenous N-linked glycosylation sites are indicated by small black, branched structures and putative diacidic motif active sites are indicated in dark blue (red for active site that has been identified in yPmts). (C) Predicted organization of human TMTC3 with signal sequence (black), hydrophobic domains (dark blue), TPR domains (orange) and predicted active site (diacidic DE and EE residues in dark blue) as designated. 
A

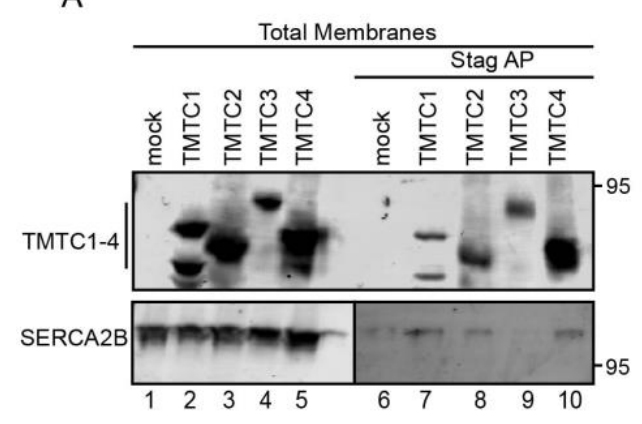

C

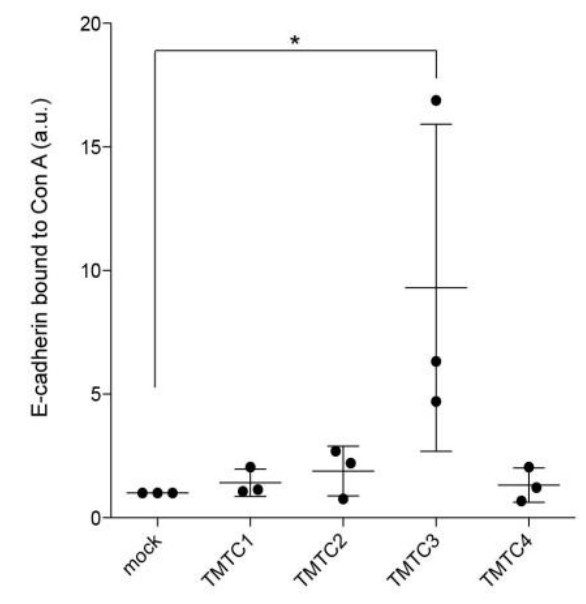

B

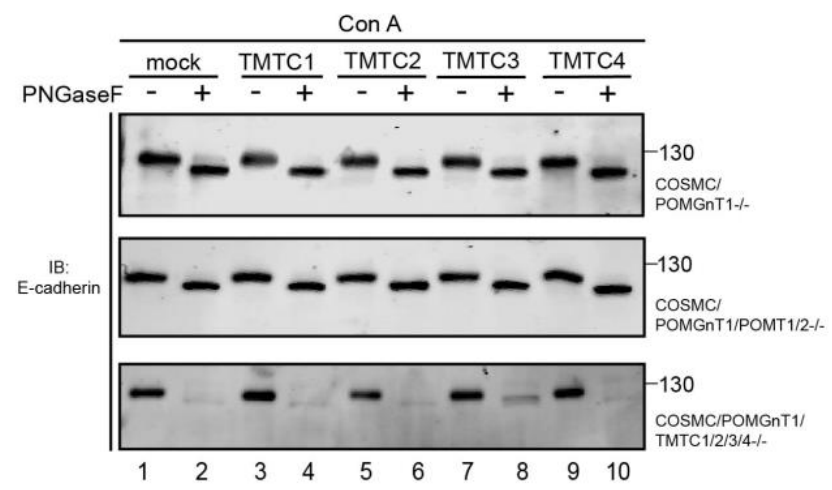

D

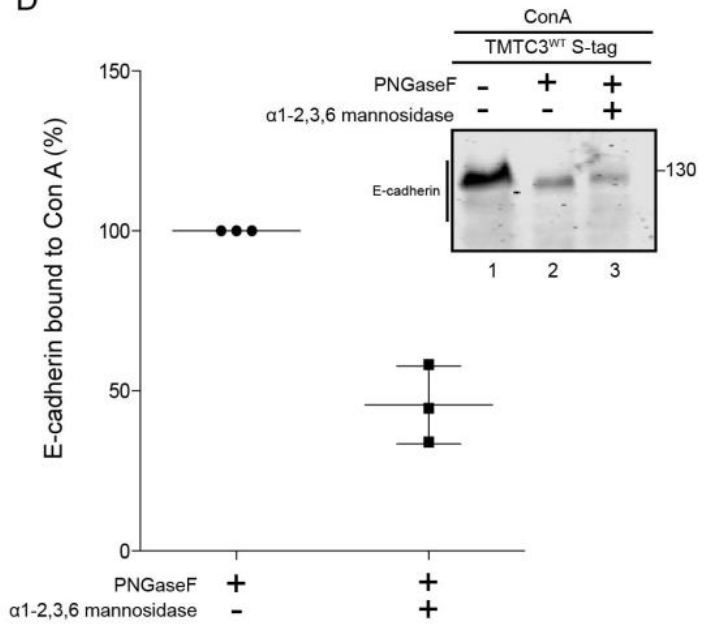

Figure 2.5. TMTC3 rescues O-mannosylation of E-cadherin in HEK293

COSMC/POMGnT1/TMTC1/2/3/4 - cells. (A) HEK293T cells expressing TMTC1, 2, 3 or 4 were harvested in isotonic buffer and homogenized. A portion of the cell homogenate was subjected to ultracentrifugation and resuspended in reducing sample buffer. This was considered the total membrane fraction (lanes 1-5). An excess of MNT lysis buffer was added to an equal amount of cell homogenate and subjected to S-protein agarose affinity purification (lanes 6-10). Proteins were detected by immunoblotting with appropriate antisera directed against the S-tag epitope and SERCA2B. (B) S-tagged TMTC1, 2, 3 or 4 cDNA was transfected into indicated cell lines. Cell lysates were collected and split, one half subjected to treatment with endoglycosidase PNGaseF (lanes 2, 4, 6, 8 and 10), prior to pulling down glycosylated proteins with Concanavalin A (Con A). Samples were then analyzed by $6 \%$ SDS-PAGE and immunoblotted with E-cadherin antisera to assess levels of glycosylated E-cadherin. (C) Quantification of relative density of O-glycosylated E-cadherin from COSMC/POMGnT1/TMTC1/2/3/4-/- cells transfected with TMTC1, 2, 3, and 4 cDNA, respectively. Statistical significance between nontransfected cells and TMTC1, 2, 3 or 4 transfected cells was calculated by using one-way 
ANOVA. Measurements designated $(*)$ have a $\mathrm{P}$ value of $<0.05$. Error bars represent standard deviation. (D) COSMC/POMGnT1/TMTC1/2/3/4-- cells were transfected with S-tagged TMTC3 cDNA. Cell lysate was collected and subjected to a glycosylation assay with either PNGaseF (lane 2) or combined PNGaseF and $\alpha 1-2,3,6$ mannosidase treatment (lane 3) prior to affinity purification of glycosylated proteins by Con A. Samples were then analyzed via 9\% SDS-PAGE and immunoblotted with E-cadherin antisera. Error bars represent standard deviation. 
A

Con A

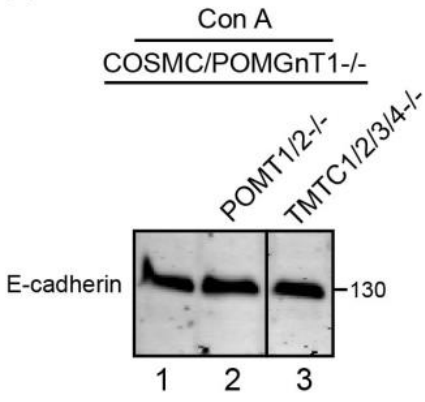

B
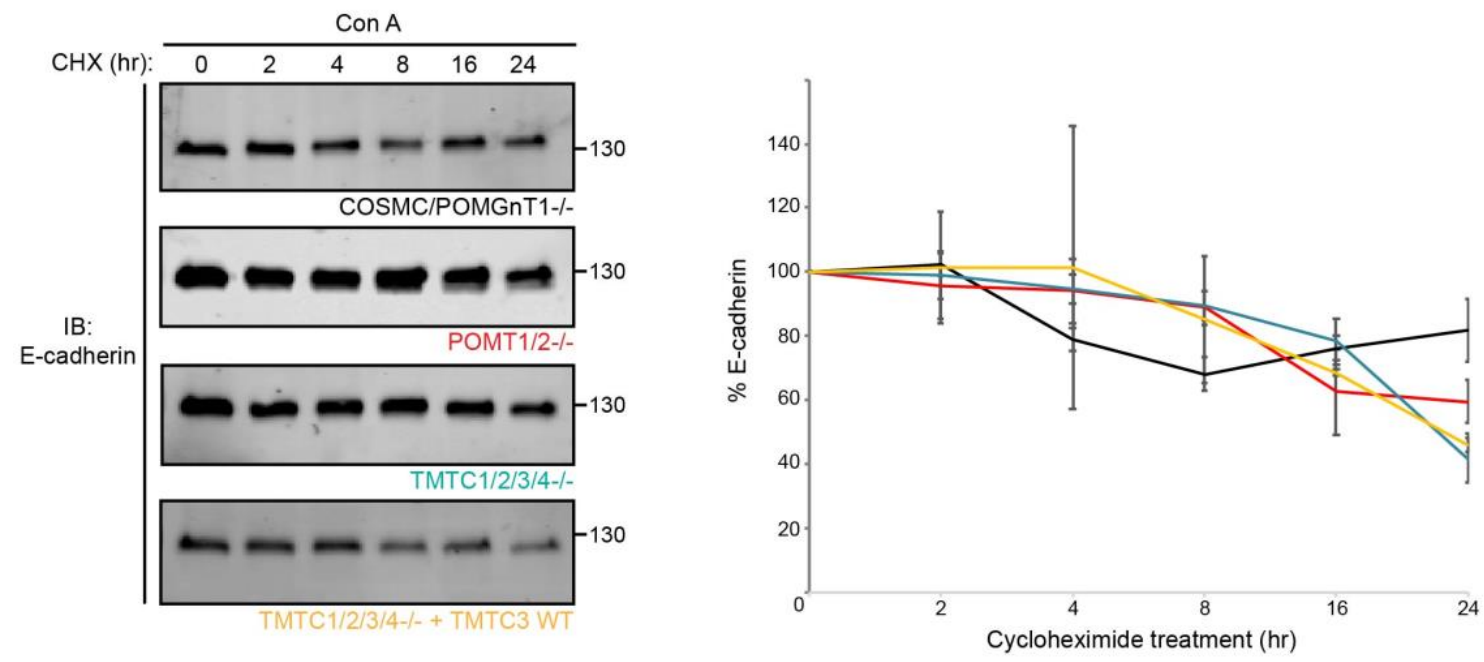

Figure 2.6. Deletion of TMTCs and E-cadherin. (A) Expression and stability of Ecadherin was assessed in O-mannosyltransferase knockout cells to determine effects of O-glycosylation. HEK293 COSMC/POMGnT1--, HEK293

COSMC/POMGnT1/POMT1/2-/- and HEK293 COSMC/POMGnT1/TMTC1/2/3/4-/- cells were lysed and total protein amount was assessed via Bradford assay. Equal amounts of protein were subjected to pull-down via Con A prior to samples being analyzed by $9 \%$ SDS-PAGE and immunoblotted with E-cadherin antisera to monitor levels of E-cadherin. (B) Previously described cell lines were treated with $100 \mu \mathrm{g} / \mathrm{mL}$ cycloheximide for indicated time $(0,2,4,8,16$ and $24 \mathrm{hr})$ prior to lysis, total protein quantification and subsequent affinity purification with Con A. Samples were then analyzed by $9 \%$ SDSPAGE (left panel) and the amount of E-cadherin remaining at 2, 4, 8, 16 and $24 \mathrm{hr}$ was quantified and normalized to the starting material $(0 \mathrm{~h})$ (right panel). Error bars represent standard error of the mean from three independent experiments. 
A

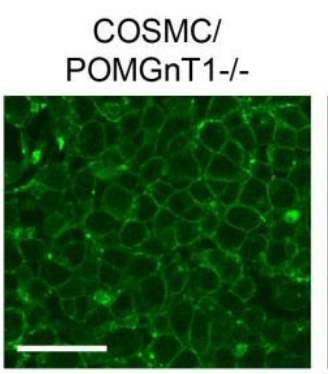

COSMC/POMGNT1 COSMC/POMGnT1

POMT1/2-/-

TMTC1/2/3/4-/-
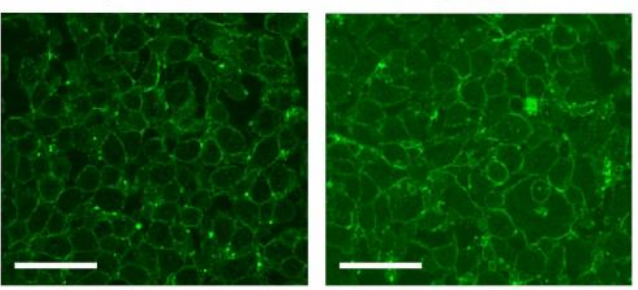

B

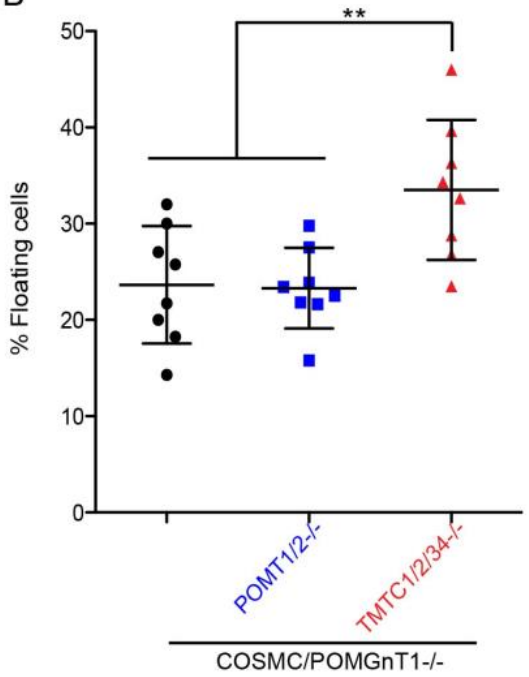

C

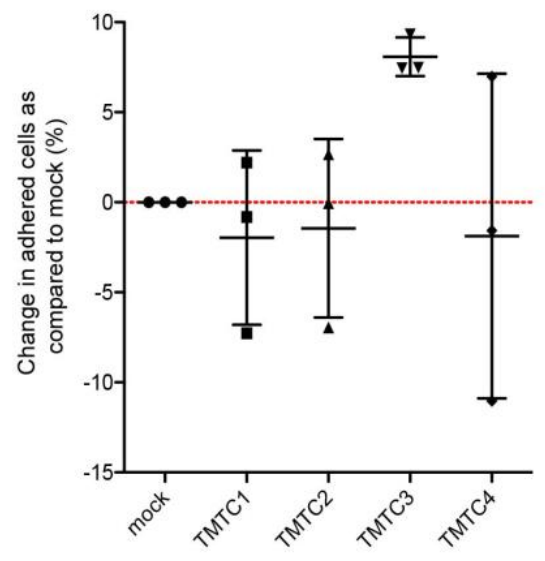

D

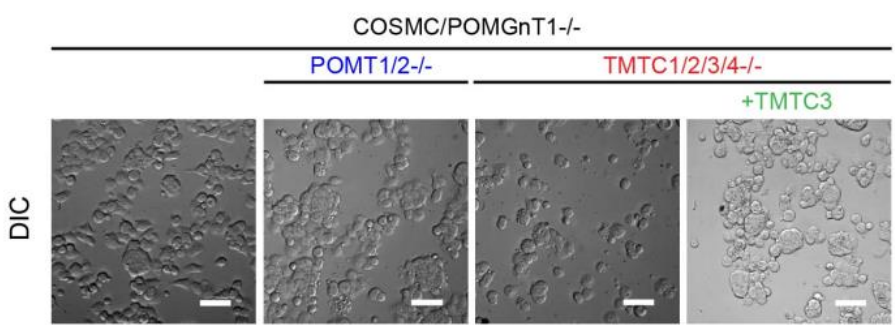

$\mathrm{E}$

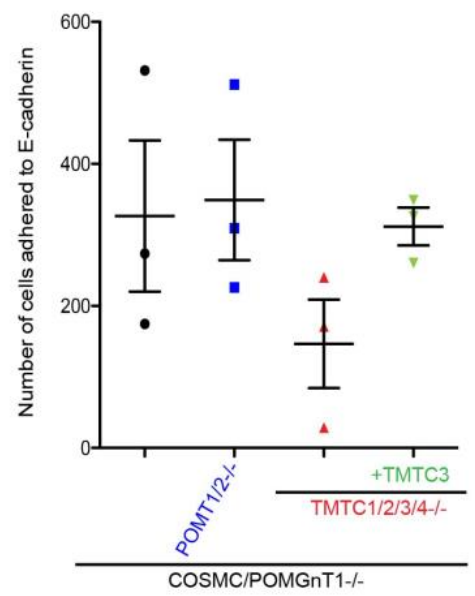

Figure 2.7. TMTC3 enhances E-cadherin mediated cellular adherence. (A) Cellular localization of cadherins was investigated by confocal microscopy. HEK293 COSMC/POMGnT1-/, COSMC/POMGnT1/POMT1/2-/- and COSMC/POMGnT1/TMTC1/2/3/4-/- cells were fixed and stained with pan-cadherin 
antisera. Scale bars correspond to $50 \mu \mathrm{m}$. (B) Adherence was assessed in previously described cell lines. Cells were resuspended in growth media and 1 x 106 cells were subsequently plated in a 24-well plate. Cells were allowed to adhere for $30 \mathrm{~min}$. Unadhered cells were collected and adhered cells were trypsinized and collected. The data describes the $\%$ of floating (unadhered) cells over the total (unadhered + adhered) after counting both fractions. Statistical significance between cell types was calculated by one-way ANOVA. Measurements designated $(* *)$ have a $\mathrm{P}$ value of $<0.01$. Error bars represent the standard deviation. (C) TMTC1, 2, 3 or 4 cDNA was transfected into COSMC/POMGnT1/TMTC1/2/3/4-- cells and adherence was assessed as described in B. The increase or decrease of adherence of cells transfected with TMTC1, 2, 3 or 4 was established by subtracting the \% of mock transfected adhered cells (red dashed line) from the $\%$ of adhered, transfected cells (adhered/ (unadhered + adhered)). Error bars represent the standard deviation. (D) Cellular adherence to immobilized E-cadherin was assessed via immunoassay. HEK293 COSMC/POMGnT1--, POMT1/2--, TMTC1/2/3/4-- cells and TMTC1/2/3/4-- cells transfected with TMTC 3 were resuspended and 60,000 cells were placed on immobilized E-cadherin $(50 \mu \mathrm{g} / \mathrm{mL})$. Cells were allowed to attach for $1 \mathrm{hr}$ prior to fixing with PFA, permeabilization with triton X-100 and staining with Hoescht. Hoescht nuclear staining and DIC images (pictured) were acquired at 40X magnification. Scale bars correspond to $50 \mu \mathrm{m}$. (E) The number of cells adhered to E-cadherin in previously described cell lines was estimated by averaging 5 randomized images capturing nuclei intensity at $405 \mathrm{~nm}$ within each well from three independent experiments. Error bars represent the standard deviation. 
A
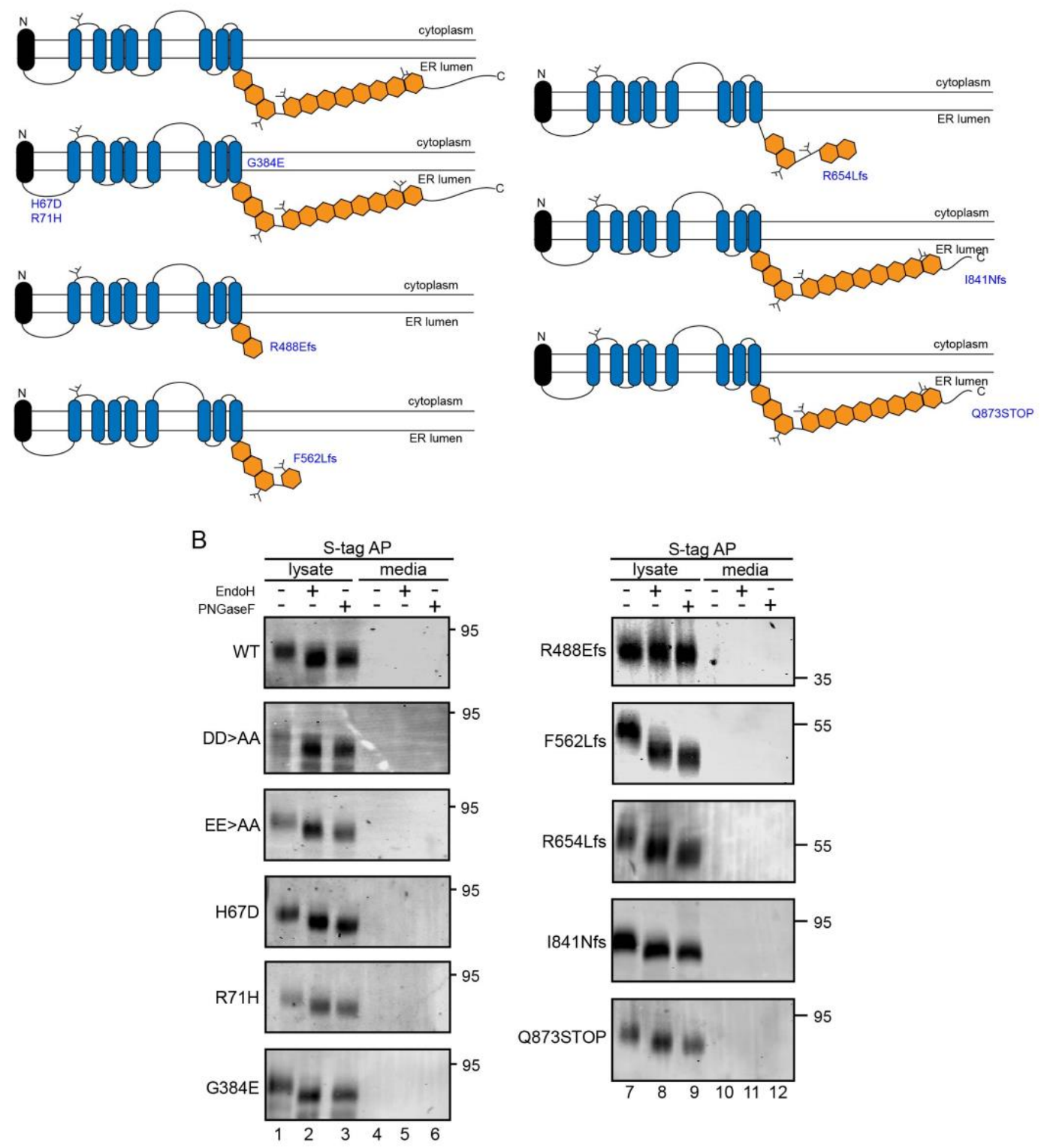

Figure 2.8. TMTC3 disease variant predictive outcomes and carbohydrate analysis. (A) The organization of TMTC3 WT and disease variants signal sequence (black), hydrophobic domains (blue), TPR domains (orange). N-linked glycosylation sites are indicated by small black, branched structures. Mutations are indicated in blue. (B) HEK293T cells were transfected with S-tagged TMTC3 WT and disease variants cDNA as indicated and were affinity purified from the media and the lysed cells using S-protein agarose. Samples were then subjected to a glycosylation assay with either EndoH (lanes 2, 5, 8 and 11) or PNGaseF (lanes 3, 6, 9 and 12) digestion as indicated. Reducing sample buffer was added, and the samples were analyzed by a $6 \%$ SDS-PAGE. 

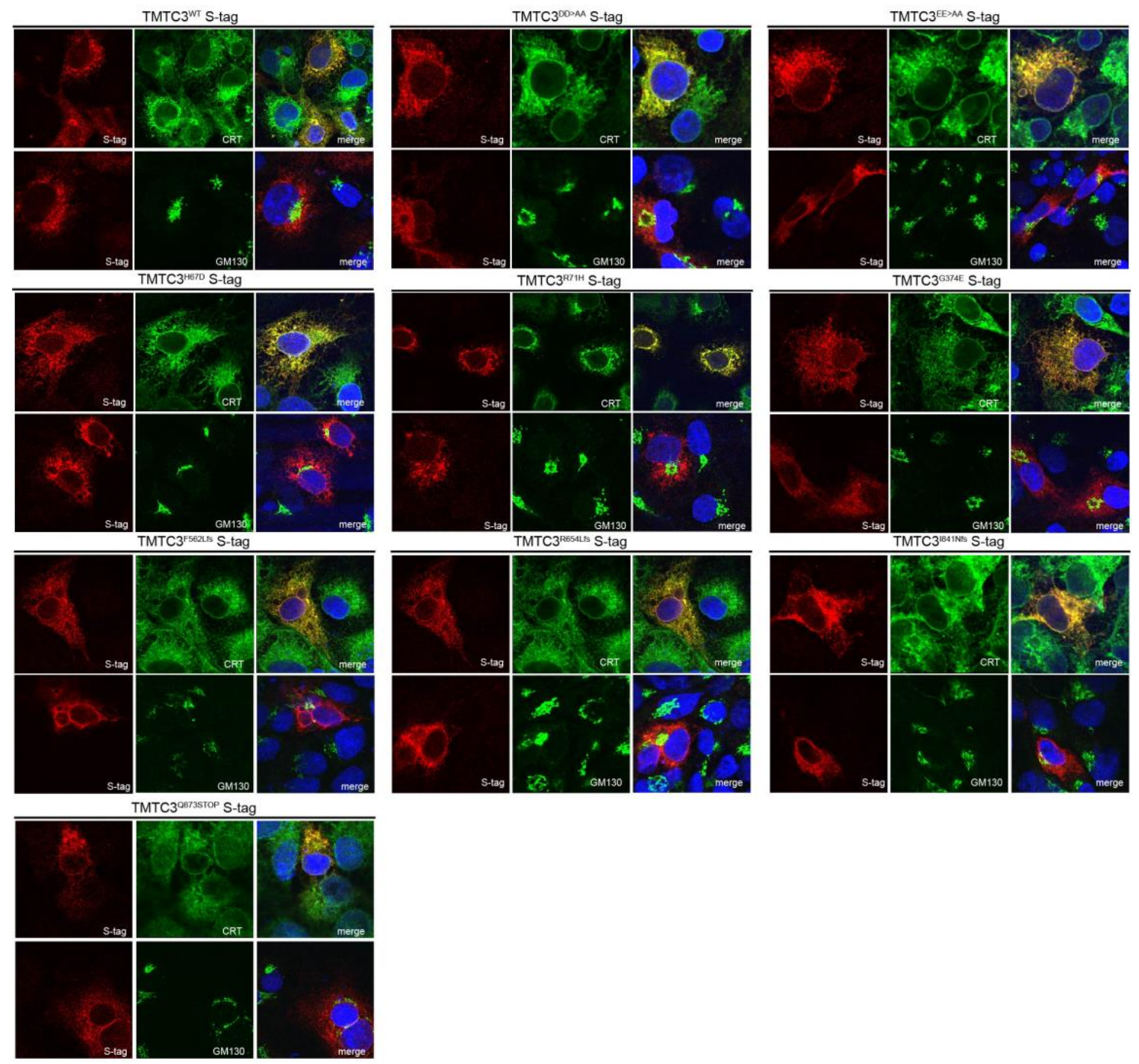

Figure 2.9. Cellular localization of TMTC3 disease variants. Cellular localization of TMTC3 disease variants was investigated by confocal microscopy. COS7 cells were transfected with TMTC3 disease variant cDNA. Fixed cells were stained with S-tag, Calreticulin (ER) or GM130 (Golgi) antisera. Nuclei were visualized by DAPI staining (blue). 
A

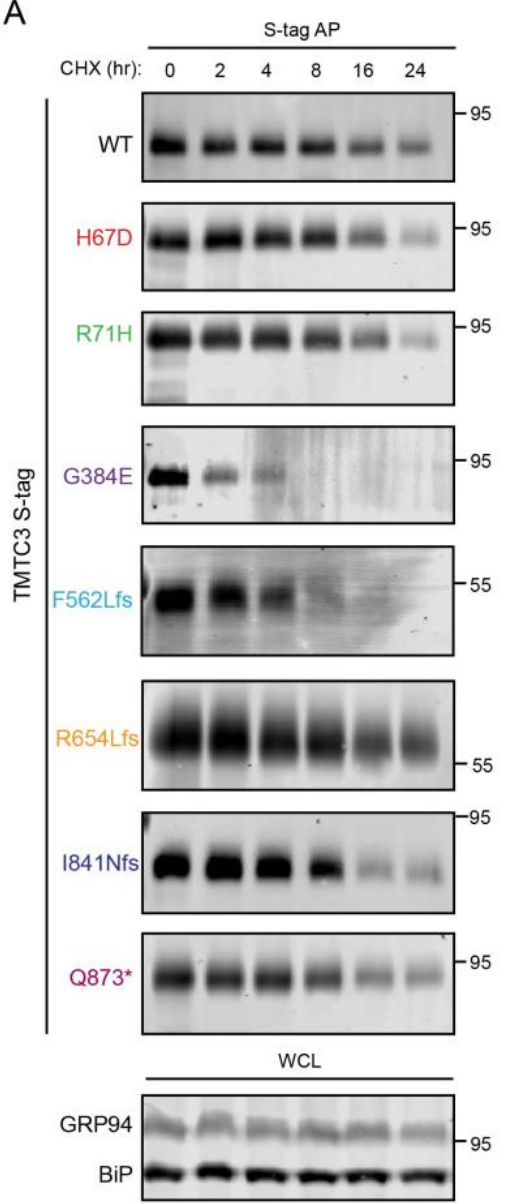

B
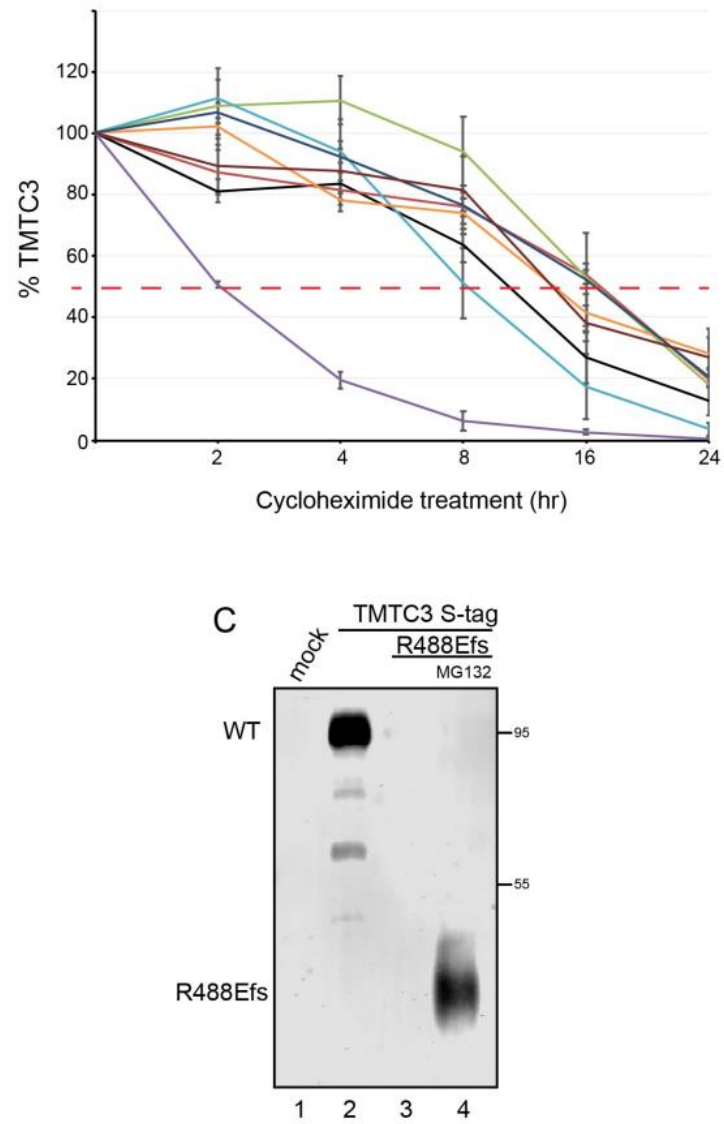

Figure 2.10. TMTC3 WT and disease variant stability. (A) HEK293T cells were transfected with S-tagged TMTC3 WT or disease variant cDNA as indicated. Cells were treated with $100 \mu \mathrm{g} / \mathrm{mL}$ cycloheximide for the indicated time prior to collection, lysed in MNT and samples were subjected to affinity purification with S-protein agarose. Reducing sample buffer was added and samples were analyzed via 9\% SDS-PAGE and immunoblotted for the S-tag epitope. Cell lysates were also subjected to precipitation with 10\% trichloroacetic acid, analyzed via 9\% SDS-PAGE and immunoblotted with KDEL antisera to determine stability of GRP94 and BiP (WCL). (B) Time points were collected at $0,2,4,8,16$ and $24 \mathrm{hr}$ after cycloheximide treatment. The amount of TMTC3 protein remaining at 2, 4, 8, 16 and $24 \mathrm{hr}$ was quantified and normalized to the starting material $(0 \mathrm{~h})$ and averaged from three independent experiments. Error bars represent standard error of the mean. (C) HEK293T cells were transfected with S-tagged TMTC3 WT or the TMTC3 R488Efs disease variant as indicated. Cells transfected with the TMTC3 R488Efs variant were treated with $20 \mu \mathrm{M}$ MG132 for $12 \mathrm{hr}$ prior to collection and lysis in MNT (lane 4). Samples were subjected to affinity purification with S-protein agarose and analyzed via SDS-PAGE prior to immunoblotting for the S-tag epitope. 
A

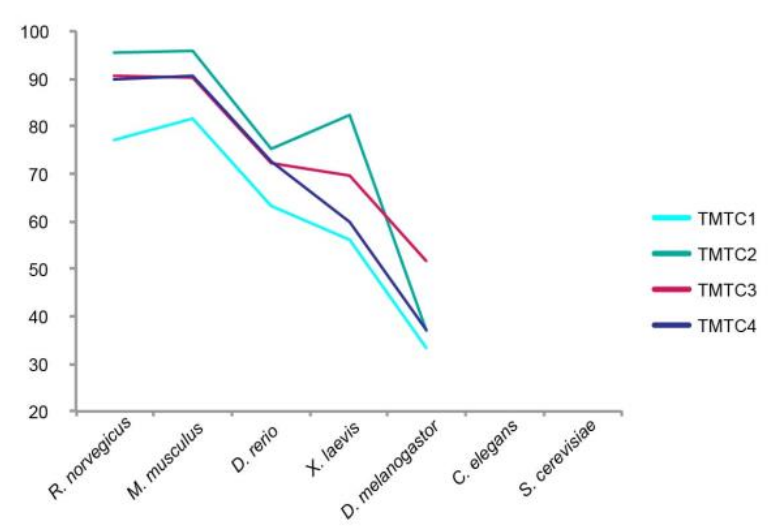

B

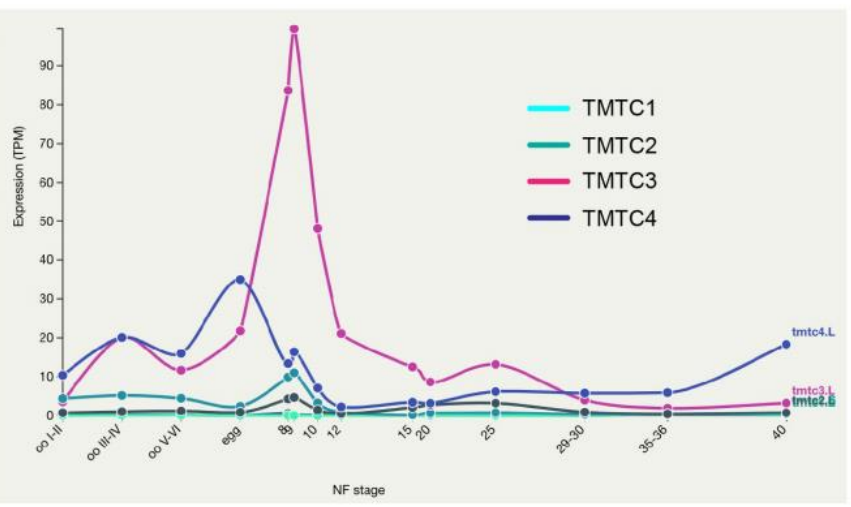

C
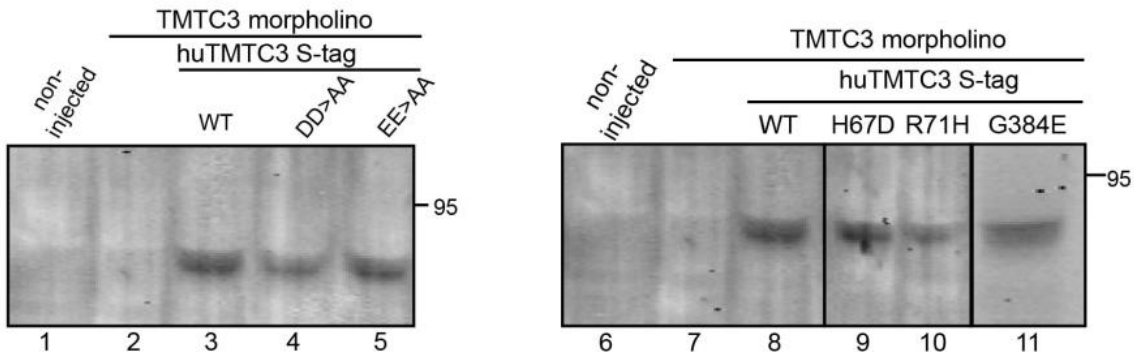
Figure 2.11. TMTC conservation in commonly studied species and RNA expression in Xenopus laevis. (A) Sequence identity (\%) of the TMTC proteins in commonly studied species was assessed by comparing amino acid sequences of each species listed to the human sequence. (B) RNA expression of TMTC1-4 during Xenopus laevis development up to stage 40. These expression profiles were based on data collected in the study by Session et. al in Nature 2016. (C) Embryos were injected with TMTC3 morpholino at the one cell stage approximately 45 min after fertilization. Selected embryos were subsequently injected with human TMTC3 RNA (lanes 3, 4, 5, 8, 9, 10 and 11). Noninjected and indicated injected embryos were collected at stage 12 and resuspended in Modified Barth's Saline (MBS) buffer with 1\% triton X-100. Total protein was precipitated with $10 \%$ trichloroacidic acid, resuspended in reducing sample buffer, analyzed by a $9 \%$ SDS-PAGE and immunoblotted for the S-tag epitope. 
A

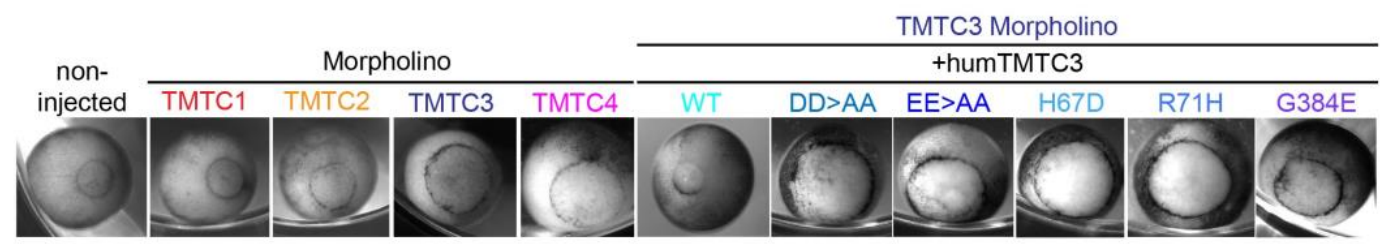

B

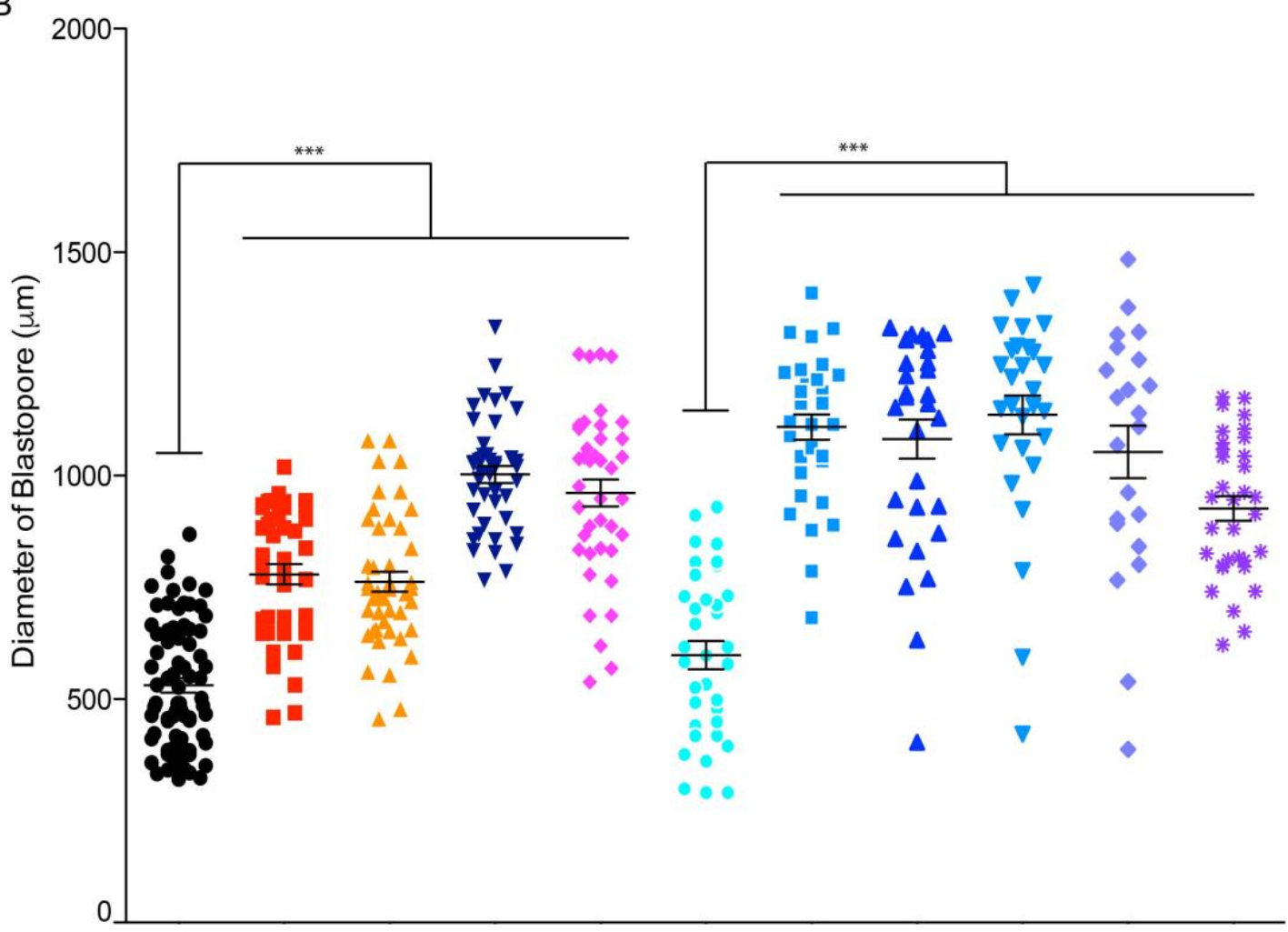

Figure 2.12. Knockdown of Tmtc3 in developing Xenopus laevis emrbyos affects gastrulation. (A) Embryos were injected with $10 \mathrm{ng}$ of morpholinos against TMTC1, TMTC2, TMTC3 and TMTC4 at the one-cell stage. The embryos were scored for gastrulation when control embryos reached stage 11-12. Embryos were also injected with $10 \mathrm{ng}$ of TMTC3 morpholino and subsequently injected with S-tagged huTMTC3WT, huTMTC3DD>AA, huTMTC3EE>AA, huTMTC3H67D, huTMTC3R71H and huTMTC3G384E at the one-cell stage. The embryos were scored as described above. (B) The average of three or more independent experiments is plotted on the graph. Statistical significance between injection conditions was calculated using one-way ANOVA. Measurements designated (***) have a $\mathrm{P}$ value of $<0.001$. The error bars represent the standard deviation to the mean. The number of embryos analyzed are as follows: non-injected n=75, TMTC1MO 
$\mathrm{n}=43$, TMTC2MO n=45, TMTC3MO n=43, TMTC4MO n=40, TMTC3MO + huTMTC3 $\mathrm{n}=35$, TMTC $3 \mathrm{MO}+$ huTMTC3DD $>$ AA $\mathrm{n}=33$, TMTC $3 \mathrm{MO}+$ huTMTC3EE $>$ AA $\mathrm{n}=30$, TMTC3MO + huTMTC3H67D $=28$, TMTC3 MO + huTMTC3R71H $\mathrm{n}=22$ and huTMTC3G384E $\mathrm{n}=33$. 
A

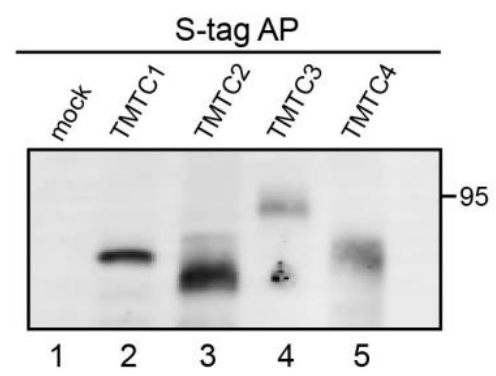

C
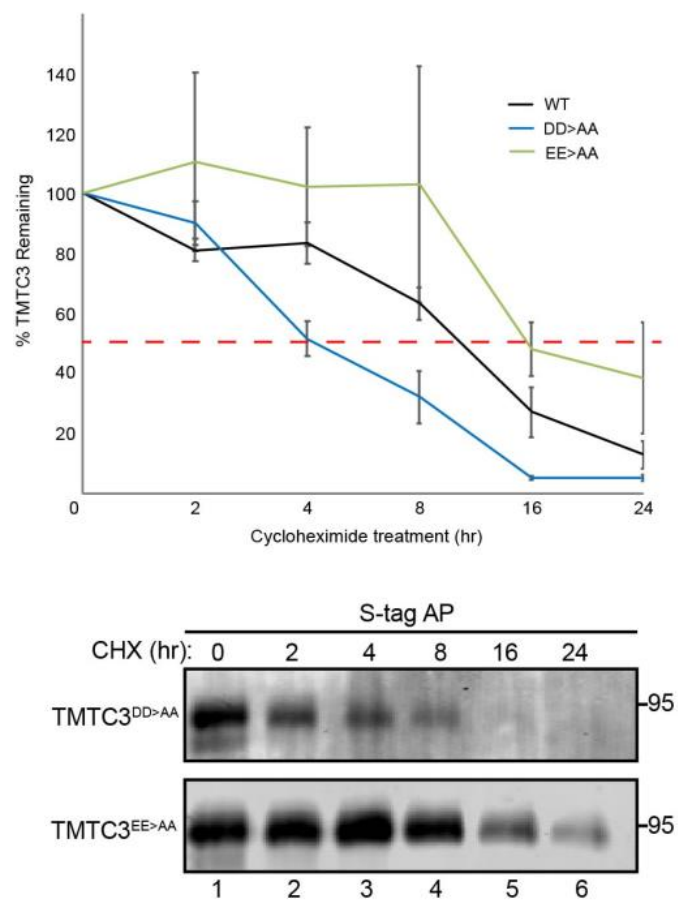

B
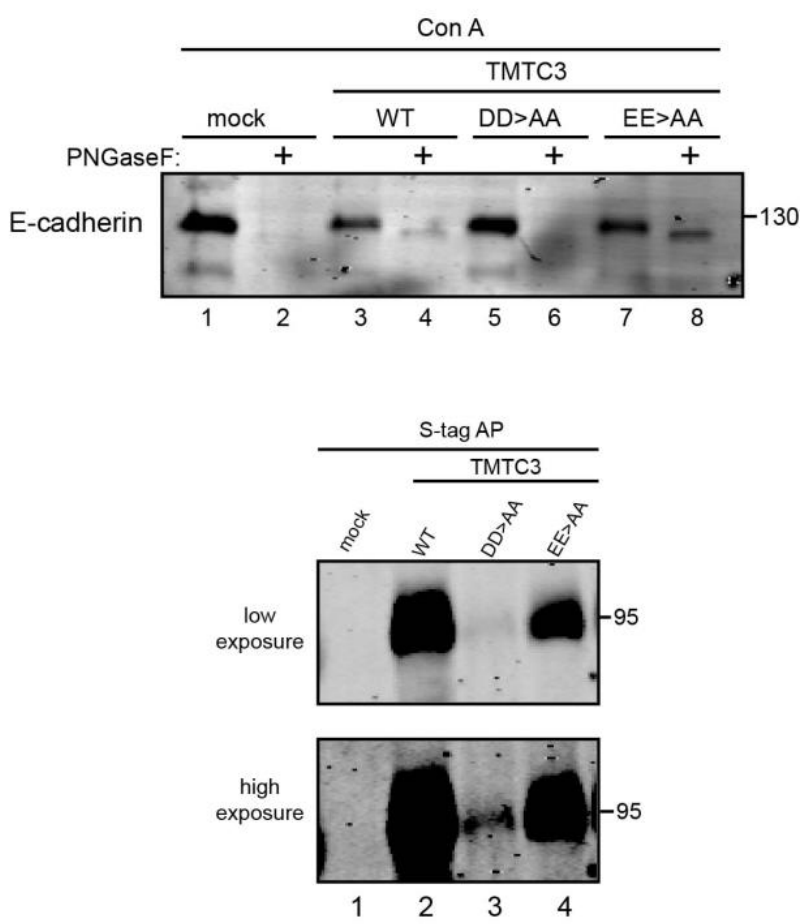

Figure 2.13. Putative active sites of TMTC3. (A) S-tagged TMTC1, 2, 3 or 4 cDNA was transfected into HEK293 COSMC/POMGnT1/TMTC1/2/3/4-/- cells. Cell lysates were collected and one third was subjected to affinity purification with $\mathrm{S}$-protein agarose.

Samples were then analyzed by 6\% SDS-PAGE and immunoblotted for the S-tag epitope to assess levels of TMTC1, 2, 3 and 4, respectively. (B) HEK293

COSMC/POMGnT1/TMTC1/2/3/4-/- cells were transfected with S-tagged TMTC3 WT or putative active site mutant (DD>AA or EE>AA) cDNA. Lysates were collected and split, one third subjected to treatment with endoglycosidase PNGaseF (lanes 2, 4, 6, 8 and 10), prior to pulling down glycosylated proteins with Con A. One third was subjected to affinity purification with S-protein agarose. Samples were then analyzed by $6 \%$ SDS- 
PAGE and immunoblotted with E-cadherin antisera (top blot) or S-tag antisera to assess levels of TMTC3 (lower blots). Lower panel represents the upper panel S-tag blot at a higher exposure to see the diminished expression of the DD>AA mutant (lane 3) (C) HEK293T cells were transfected with S-tagged TMTC3 WT or putative active site mutant cDNA as indicated. Cells were treated with $100 \mu \mathrm{g} / \mathrm{mL}$ cycloheximide for the indicated time prior to collection in lysis buffer. Samples were subjected to affinity purification with S-protein agarose and analyzed via 9\% SDS-PAGE and immunoblotted for the S-tag epitope. Time points were collected at $0,2,4,8,16$ and $24 \mathrm{hr}$ after cycloheximide treatment. The amount of TMTC3 protein remaining at 2, 4, 8, 16 and 24 $\mathrm{hr}$ was quantified and normalized to the starting material $(0 \mathrm{~h})$ and averaged from three independent experiments. Error bars represent standard error of the mean (upper panel). 


\section{CHAPTER 3}

\section{TTC17 IS A NOVEL ER ADAPTOR INVOLVED IN ER STRESS}

\subsection{Abstract}

The endoplasmic reticulum (ER) is a complex, multi-functional organelle, comprised of a continuous membrane and lumen that is organized into several functional regions with various roles including protein translocation, folding, quality control, secretion and the unfolded protein stress response (UPR). Total cellular protein homeostasis is maintained by a complicated chaperone network. Bioinformatic categorization of the various chaperome factors into functional families revealed that the largest family consists of proteins containing tetratricopeptide repeats (TPRs). TPRs are well-studied structural motifs that support intermolecular protein-protein interactions. TTC17 was identified by an in silico search for TPR-containing proteins that are predicted to possess an ER targeting signal sequence. Confocal microscopy and carbohydrate analysis revealed that TTC17 acquires a number of N-linked glycans and resides in the ER. TTC17 was determined to be a soluble protein within the ER lumen by alkaline extraction and trypsin protection assays performed on ER-derived microsomes. Immunofluorescence staining and immunoblotting of endogenous TTC17 found it to be highly up-regulated in cells that also show higher expression levels of ER chaperones BiP, GRP94 and GRP170. Transcript regulation analysis demonstrated that it is significantly up-regulated by chemically induced ER stress and cellular protein levels were markedly increased in a mutant cell line that undergoes adaptive stress and is grown at an elevated temperature. Since TTC17 lacks an ER retention sequence, it is likely retained in the ER by associating with other ER resident proteins. Proteomic analysis 
uncovered interactions between a C-terminally tagged, overexpressed TTC17 and a number of ER resident chaperones. Given that TTC17 is regulated by ER stress as are many of the chaperones it putatively interacts with, it is possible that TTC17 acts as an adaptor protein to spatially and/or temporally coordinate chaperones under cellular stress conditions.

\subsection{Introduction}

The endoplasmic reticulum (ER) is an essential compartment for protein biosynthesis and maturation within the cell (Ni and Lee, 2007). It also functions as the major storage organelle for $\mathrm{Ca} 2+$ and lipid biogenesis. Given the various, essential cellular functions that it performs and being comprised of a continuous membrane envelope and lumen, it is compartmentalized into numerous functional regions (Graham et al., 2019; Lynes and Simmen, 2011). The organization of the ER is directed by extrinsic factors that connect the ER to the nuclear envelope, the cytoskeleton, other organelles and plasma membrane and by ER resident adaptor proteins (Graham et al., 2019; Levine and Loewen, 2006; Voeltz et al., 2002). The adaptor proteins nucleate the formation of large protein complexes supporting the compartmentalization of the ER (Carvalho et al., 2006; Christianson et al., 2008; Mueller et al., 2008). This organization contributes to the ER's ability to efficiently perform functions in the maturation, quality control and trafficking of secretory pathway cargo, calcium regulation and lipid biogenesis (English et al., 2009; Hebert and Molinari, 2007; Lynes et al., 2013). The ER is essential to maintaining cellular homeostasis, however many questions still remain as to how the organization of the ER is maintained. 
Tetratricopeptide repeat (TPR) protein structural motifs support protein-protein interactions and the nucleation of multi-protein complexes (D'Andrea and Regan, 2003; Zeytuni and Zarivach, 2012). TPRs are universally found in all organisms and diverse pathways. A TPR motif consists of 34 amino acids that fold into two anti-parallel alpha helices (Figure 1.1A). There is no consensus sequence for the TPR and no residue is in invariant, however, there is a preference for certain amino acids at defined positions (D'Andrea and Regan, 2003). TPR motifs can be found in tandem arrays of 2-16 sequential motifs (D'Andrea and Regan, 2003; Jiménez et al., 2012). Clusters of three TPR tandem motifs are the most common and frequently bind more specific ligands, whereas longer stretches of sequential TPRs commonly exhibit more promiscuous binding. Adjacent TPR units form a series of repeating antiparallel $\alpha$-helices, yielding an overall super-helix structure (Zeytuni and Zarivach, 2012). This super-helix fold forms a pair of concave and convex surfaces, which display amino acid variety. This allows TPRcontaining proteins to use different binding surfaces to interact with ligands thus contributing to their diversity as a protein-protein interaction motif. The TPR functions in a variety of proteins involved in numerous cellular processes such as gene regulation, mitosis, regulation of steroid receptor function, virulence, protein import and protein homeostasis (Cerveny et al., 2013; Graham et al., 2019).

A database search predicted that the human genome encodes $\sim 280$ proteins containing TPR motifs (Letunic and Bork, 2018, 2019). Further bioinformatic analysis combining extensive literature on biochemical properties of chaperones/co-chaperones and protein domain identifier databases to examine the expression of genes encoding molecular chaperones revealed that $\sim 41 \%$ of the predicted TPR-containing proteins 
contribute to maintaining the cellular proteostasis network (Brehme et al., 2014). Among the 114 TPR-containing proteins predicted to be co-chaperones, eight of the nine identified ER localized proteins are included (Figure 1.2). The list excludes SEL1L most likely due to the difference in TPR consensus sequence length (Mittl and SchneiderBrachert, 2007). Additionally, ERdj6 is included in the Heat Shock Protein (HSP)40 category as the groups were prioritized by chaperone categories rather than localization (Brehme et al., 2014). TMTC1-4, TTC13 and TTC17 are included in the TPR cochaperone family although they have not yet been shown to function as co-chaperones nor their cellular localization been determined. Until recently, SEL1L, ERdj6 and FICD were the only ER TPR-containing proteins characterized in mammalian cells (Gardner et al., 2000; Rutkowski et al., 2007; Sanyal et al., 2015) and all three proteins are critical for maintaining ER proteostasis as they are up-regulated under stress conditions and interact with the ER HSP70 paralog, BiP (Christianson et al., 2008). TMTC1-4 are novel transmembrane TPR-containing proteins most recently shown to localize to the ER (Li et al., 2018; Racapé et al., 2011; Sunryd et al., 2014). They have been suggested to function as O-mannosyltransferases of the cadherin family of proteins and shown to be involved in calcium regulation. Deletion of Tmtc4 in mice has also been shown to activate the unfolded protein response (UPR) (Li et al., 2018).

Any disruption of ER function, including $\mathrm{Ca} 2+$ dysregulation, metabolic disorder like hypoxia, viral infection or inhibition of protein folding like glycosylation or disulfide bond formation, results in the accumulation of unfolded or misfolded proteins $(\mathrm{Ni}$ and Lee, 2007). This leads to ER stress, which is defined as an imbalance between cellular demand for ER function and ER capacity. In order to reduce excessive misfolded protein 
stress, the cell triggers the UPR. This initiates transient attenuation of protein translation, degradation of misfolded proteins as well as the induction of molecular chaperones and folding enzymes to augment the ER capacity for protein folding and degradation. The ER contains numerous molecular chaperones that are physiologically involved in posttranslational modification, disulfide bond formation, folding assembly and quality control of newly synthesized proteins to preserve cellular homeostasis. Upon ER stress, the upregulation of these proteins is pivotal for cell survival.

Here, an in silico approach was used to expand our understanding of ER adaptors that participate in the organization of the ER using TPR motifs. TTC17 was identified as a TPR-containing protein that possesses a potential N-terminal ER targeting sequence. Characterization of endogenous TTC17 and transiently expressed S-tagged TTC17 found it to be a soluble protein that resides in the ER lumen. Examination of its transcript regulation revealed that $T T C 17$ is significantly up-regulated under ER stress conditions at similar levels of previously characterized ER TPR-containing proteins, SEL1L, ERdj6 and FICD. Furthermore, endogenous TTC17 is up-regulated under heat stress in cells that display an adaptive ER stress response. A shot-gun proteomics approach was employed that identified numerous ER chaperones as potential interacting partners of TTC17, indicating that it may act as a scaffold adaptor to spatially regulate major molecular chaperones. Collectively, these findings showed that TTC17 is a novel TPR-containing adaptor protein involved in ER stress.

\subsection{Results}

\subsubsection{TTC17 is an ER resident protein}


As adaptor proteins commonly use clusters of TPR motifs to modulate proteinprotein interactions and TPR-containing proteins are implicated heavily in co-chaperone function, we hypothesize that the ER might contain TPR-containing proteins, in addition to SEL1L, ERdj6, FICD and TMTC1-4, that contribute to chaperone organization. In silico analysis, using SignalP4.0, TargetP1.1, $\Delta \mathrm{G}$ and domain architecture database SMART7, indicated that TTC17 (NCBI Accession \#NP_060729.2 (http://www.ncbi.nlm.nih.gov/protein/NP_060729.2)) contained a potential N-terminal signal sequence and eight TPR motifs (Figure 3.1A) (Emanuelsson et al., 2000; Hessa et al., 2007; Letunic and Bork, 2018; Nielsen, 2017; Nielsen et al., 1997, 2019).

Cellular localization of endogenous TTC17 was determined by a glycosylation assay and immunofluorescence confocal microscopy. Secretory proteins are commonly modified in the ER with N-linked glycans at the consensus site Asn-Xxx-Ser/Thr. TTC17 possesses twelve (Homo sapien, human) or eleven (Cricetulus griseus, hamster) predicted N-linked glycosylation consensus sites, respectively (Figure 3.1A), therefore a glycosylation assay was used to further analyze ER targeting and localization (Gupta, 2002). As the molecular weight of an $\mathrm{N}$-linked glycan is $\sim 2.5 \mathrm{kDa}$, the removal of $\mathrm{N}$ linked glycans by glycosidase treatment results in a corresponding increase in mobility for the deglycosylated protein. Endoglycosidase $\mathrm{H}$ (Endo $\mathrm{H}$ ) trims the high mannose glycans encountered in the ER while Peptide-N-Glycosidase F (PNGase F) removes complex glycans acquired in the Golgi in addition to high mannose glycans.

Wild type (WT) chinese hamster (Cricetulus griseus) ovary (CHO) cell lysate and media fractions were collected and subjected to glycosidase treatment. A shift upon PNGaseF treatment (Figure 3.1B, lane 3) was observed for TTC17 indicating that it was 
targeted to the ER and received N-linked glycans. A similar increase in mobility was observed upon Endo $\mathrm{H}$ treatment (Figure 3.1B, lane 2) demonstrating that the carbohydrates were high mannose glycoforms suggesting that TTC17 is an ER resident protein (Figure 3.1B).

To biochemically characterize human TTC17, cDNA was subcloned into a mammalian expression vector harboring a C-terminal S-tag, and its localization was assessed by the same methods. HEK293T cells were transfected with TTC17 S-tag, and cell lysate and media fractions were affinity precipitated with S-protein agarose beads followed by glycosidase treatment. A shift upon PNGaseF treatment (Figure 3.1C, lane 3) was observed for TTC17 S-tag indicating that it was targeted to the ER and received Nlinked glycans. A similar increase in mobility was observed upon Endo $\mathrm{H}$ treatment (Figure 3.1C, lane 2) signifying that the carbohydrates were high mannose glycoforms consistent with human S-tagged TTC17 being an ER resident protein (Figure 3.1C). WT CHO cells were immunostained for endogenous TTC17 and staining was compared against an ER (KDEL) or Golgi (GM130) marker (Figure 3.1D). TTC17 colocalized with ER marker, KDEL, while co-localization was not observed with GM130 (Figure 3.1D). C-terminally S-tagged human TTC17 was similarly assessed for localization and also co-localized with KDEL and not GM130 (Figure 3.1E). Therefore, the glycosylation profiles and the cellular distribution are consistent with both human and hamster TTC17 residing in the ER.

\subsubsection{TTC17 is a soluble protein that resides within the ER lumen}

Analysis of the TTC17 protein sequence with $\Delta \mathrm{G}$ prediction demonstrated that it does not contain hydrophobic segments, thus predicting it to be a soluble protein within 
the ER lumen (Figure 3.1A). Alkaline extraction and a protease protection assay were performed on isolated membrane fractions to determine if TTC17 is a soluble protein residing within the ER lumen (Mostov et al., 1981).

WT CHO cells were homogenized in isotonic buffer and fractions were separated by centrifugation. TTC17 was found enriched in the whole cell lysate (WCL) and total membrane (TM) fractions (Figure 3.2A, lanes 1 and 4). Alkaline extraction of the total membrane fraction followed by centrifugation found TTC17 enriched in the supernatant fraction (S) (Figure 3.2A, lane 5). This profile was observed for the ER soluble protein, Glucosidase II $\alpha$, and not the membrane bound protein calnexin, which appeared exclusively in the pellet (P) (Figure 3.2A, lanes 5 and 6). Therefore, TTC17 is a soluble protein.

Additionally, a protease protection assay was employed to confirm that TTC17 resides in the ER lumen. First, alkaline extraction was performed as previously described in HEK293T cells expressing human TTC17 S-tag to show that it was also a soluble protein (Figure 3.2B, lane 5). Next, HEK293T cells were transfected with a human TTC17 construct containing a C-terminal S-tag, and cells were homogenized prior to isolation of ER-enriched microsomes. Isolated microsomes were resuspended in an isotonic buffer, and aliquots were treated with triton $\mathrm{X}-100$ and the protease, trypsin, as indicated. Trypsin treatment did not appear to cleave TTC17 (Figure 3.2C, compare lane 1 to 3 ). As the S-tag is at the C-terminus, this demonstrated that TTC17 was positioned in the ER lumen. Combined with the modification of the glycosylation sites (Figure 3.1B and C), these results demonstrated that TTC17 is a soluble ER protein.

\subsubsection{TTC17 is upregulated by folding, calcium, trafficking and proteasomal stress}


Proteins that reside in the secretory pathway are frequently transcriptionally upregulated by stress (Walter and Ron, 2011). Of the nine TPR-containing proteins that localize to the ER, four have been shown to be transcriptionally up-regulated by stress; including SEL1L, ERdj6, FICD and TMTC4 (Christianson et al., 2008; Li et al., 2018; Rutkowski et al., 2007; Sanyal et al., 2015). To determine whether TTC17 is transcriptionally regulated by ER stress, HEK293A cells were exposed to different ER stress conditions induced by various pharmacological compounds. Cells were subjected to $\mathrm{N}$-glycan synthesis inhibition (tunicamycin), calcium depletion (thapsigargin), redox stress (dithiotreitol, DTT), inhibition of anterograde protein trafficking (brefeldin A) or proteasomal inhibition (MG132). RNA was harvested from cells followed by reverse transcription to generate cDNA and changes in gene expression were measured by qRTPCR.

TTC17 gene expression was significantly increased with tunicaymycin, thapsigargin, brefeldin A and MG132 treatment by 7.1, 4.6, 8.9 and 2.4-fold, respectively (Figure 3.3B). TTC17 was also up-regulated $\sim 1.6$-fold by DTT. BiP was significantly stimulated by all treatments at $\sim 10.7,82.3,4.9,24.5$ and 12.5-fold for tunicamycin, thapsigargin, DTT, brefeldin A and MG132 treatment, respectively. In addition, the assessment of basal mRNA levels revealed that TTC17 mRNA is expressed at $\sim 1.3 \%$ of the reference gene $\beta$-actin whereas ER resident chaperone, $B i P$, is $\sim 20 \%$ of $\beta$-actin (Figure 3.3A). As expected, BiP basal mRNA levels are far more elevated than TTC17 as $\mathrm{BiP}$ is one of the most abundant proteins in the ER and is tightly regulated to maintain cellular homeostasis (Pobre et al., 2018). Interestingly, SEL1L, ERdj6 and FICD have similar basal mRNA expression to TTC17 and are also shown to be significantly 
induced by ER stress (Figure 3.3B). TTC17 is significantly up-regulated by several forms of stress like these ER co-chaperones and ERAD components, suggesting that it may be involved in the ER stress response as the other three proteins have been shown to be (Rutkowski et al., 2007; Sanyal et al., 2015; Sun et al., 2014).

\subsubsection{Endogenous TTC17 and ER chaperones show elevated expression levels in cells}

$\mathrm{BiP}$ is one of the most abundant proteins in the ER, along with the ER Hsp90 paralog, GRP94, collagen specific chaperone HSP47, cyclophilin B, lectin binding chaperone calreticulin, PDI and ERp57 (Gidalevitz et al., 2013). The ER chaperones can be categorized into three groups: (1) chaperones of the heat shock protein (HSP) family including GRP78/BiP, GRP94 and their co-chaperones; (2) chaperone lectins like calnexin, calreticulin and EDEMs; and (3) substrate-specific chaperones such as HSP47 (Ni and Lee, 2007). Given that TTC17 was identified as a likely co-chaperone within the largest functional family of the cellular chaperone network, we examined its expression in cells by immunofluorescence confocal microscopy (Brehme et al., 2014).

WT CHO cells were immunostained for endogenous TTC17 and staining was compared against endogenous BiP, GRP94 and co-chaperone/nucleotide exchange factor, GRP170 (Figure 3.4). TTC17 co-localized with each protein, and interestingly showed similar elevated expression levels (Figure 3.4). By observation, 1-5\% of cells showed elevated expression of these chaperone family members suggesting that TTC17 along with BiP, GRP94 and GRP170 are conditionally regulated amongst a subpopulation of cells. 
In order to determine why these proteins were elevated in a low percentage of cells, immunostaining was performed on cells under a number of conditions and any significant change in expression of TTC17 was noted. BiP and GRP94 are not only upregulated during protein folding stress, but they are up-regulated under a number of cellular stresses including calcium dysregulation, during cell cycle check points and signal transduction (Adomako et al., 2015; Dudek et al., 2009). Cells were treated with the pharmacological ER stress inducer compounds including tunicamycin (N-glycan synthesis inhibition), thapsigargin (calcium depletion), dithiotreitol, DTT (redox stress), brefeldin A (inhibition of anterograde protein trafficking) or MG132 (proteasomal inhibition) (Figure 3.3B) as well as compounds that synchronized the cells in the G0 phase of the cell cycle (retinoic acid), serum starvation and amino acid starvation. Change in TTC17 expression was not observed under any of these treatments (data not shown). Interestingly, when we assessed TTC17 expression in a genetically manipulated CHO cell line (MI8-5) that undergoes adaptive stress, a significant increase was observed (Figure 3.5A, lower panel). MI8-5 cells are mutagenized $\mathrm{CHO}$ cells that resulted from a mannose suicide selection screen using temperature sensitivity (Quellhorst et al., 1999). MI8-5 cells were shown to produce unglucosylated N-linked glycans, resulting from a mutation in the ALG6 gene. Normally, N-linked glycans are assembled prior to en bloc transfer of a Glc3-Man9-GlcNAc2 carbohydrate branch to a translocating polypeptide by the oligosaccharyltransferase (OST) complex (Tannous et al., 2015). The glycans in MI85 cells are successfully transferred onto folding secretory proteins, however, they were missing the three terminal glucoses. The terminal glucoses are critically important for engaging carbohydrate folding chaperones calnexin and calreticulin during folding (Aebi 
et al., 2010). As glycoproteins could not initially engage this chaperone system for productive folding, ER stress was induced (Pearse et al., 2008). Interestingly, the cells continued to grow under adaptive stress at the permissive temperature of $34{ }^{\circ} \mathrm{C}$, whereby the UPR is continually induced as XBP-1 is cleaved, but chaperone levels are not upregulated over WT.

The original selective mannose screen for MI8-5 cells used temperature sensitivity to select for defects at an elevated temperature $\left(40.5^{\circ} \mathrm{C}\right)$, however, MI8-5 cells did not survive at this temperature. Therefore, TTC17 expression was assessed at $37^{\circ} \mathrm{C}, 3$ ${ }^{\circ} \mathrm{C}$ higher than their normal growth temperature. First, transcript levels of TTC17 were determined in WT CHO and MI8-5 cells at different growth temperatures (Figure 3.5B). RNA, harvested from cells grown under normal conditions, was reverse transcribed to generate cDNA and changes in gene expression were measured by qRT-PCR. TTC17 was elevated in both WT CHO and MI8-5 cells grown at $37^{\circ} \mathrm{C}$ by $\sim 2.2$ and $1 \%$ higher, respectively, than $T T C 17$ in cells grown at $34^{\circ} \mathrm{C}$ (Figure 3.5B). Both immunostaining and immunoblotting showed significantly elevated expression of TTC17 in MI8-5 cells grown at $37^{\circ} \mathrm{C}$ (Figure 3.5A, C and D). The immunoblots and immunofluorescent images also revealed slightly elevated levels of the ER HSP chaperones, BiP and GRP94 (Figure 3.5 A and C). Based on this data, there appears to be a correlation between the elevated expression of chaperones and TTC17, however further analysis is required to understand the relationship.

\subsubsection{TTC17 interacts with a number of chaperones}

To identify binding partners of TTC17, a shot-gun liquid chromatography-mass spectrometry/mass spectrometry (LC-MS/MS) approach was used. HEK293T cells 
expressing TTC17 with a C-terminal S-tag were homogenized and ER-derived microsome fractions were isolated. Proteins associated with TTC17 were isolated using the S-protein agarose beads and resolved by SDS-PAGE. The gels were subjected to either silver staining or in-gel trypsin digestion followed by LC-MS/MS analysis (Figure 3.6A). The proteins associated with S-tagged TTC17 obtained from the LC-MS/MS analysis are listed in Figure 3.6C. The LC-MS/MS results indicated possible interactions for TTC17 with $~ 8$ chaperones, including GRP94 and BiP.

To verify the interactions identified using the mass spectrometry approach, HEK293T cells were transfected with C-terminal S-tagged TTC17, followed by affinity purification and immunoblotting against endogenous associated proteins. Although an interaction with the ER HSP chaperones was not validated via immunoblotting, a transient interaction could still take place, but further analysis is required. Interestingly, TTC17 interacted with glycoprotein folding sensor UDP-glucose:glycoproteinglucosyltransferase (UGGT1) (Figure 3.6B, lane 4) as did other S-tagged UPR induced ER proteins TMTC4, SEL1L and EDEM1 (Figure 3.6B, lanes 5, 6 and 7). The interaction between TTC17 and UGGT1 could be due to the extensive glycosylation of TTC17. UGGT1 acts a gatekeeper of the secretory pathway quality control system (ERQC), querying folding substrates for secretion if deemed correctly folded or re-glucosylation of their N-linked glycans so they may re-engage the carbohydrate binding chaperones calnexin and calreticulin (Tannous et al., 2015). TTC17 is a large protein that is heavily glycosylated, thus it may engage UGGT1 for folding help but further investigation is necessary.

\subsection{Discussion}


Here, we identified and characterized a novel TPR-rich ER protein, TTC17. Our data shows that TTC17 is an extensively glycosylated, soluble ER resident protein that is transcriptionally up-regulated by ER stress. Endogenous expression was also shown to be elevated in a small percentage of cells and this expression level corresponded to elevated levels of ER chaperones, BiP, GRP94 and GRP170. The percentage of cells displaying up-regulated levels of TTC17 and chaperones increased significantly in a constitutively stressed mutant cell line, MI8-5. Furthermore, it was determined that growing the mutant cells at a higher temperature, possibly inducing heat stress, contributed to the increased expression of TTC17. Proteomic analysis of affinity precipitated TTC17 protein complexes showed that TTC17 putatively interacts with a number of ER resident chaperones, including $\mathrm{BiP}$ and GRP94. Together, this data along with bioinformatic analysis suggests that TTC17 may act as a classic TPR co-chaperone to ER resident chaperones, regulating their organization or function under ER stress conditions.

Basic Local Alignment Search Tool (BLAST) analysis indicated that TTC17 homologues are conserved in the chordata phylum, however, they are absent in lower eukaryotes, such as yeast, and RNA-sequencing data shows that it is expressed in a number of tissues (Brini and Carafoli, 2009; Uhlén et al., 2015). Published data has demonstrated that TTC17 interacts with C2orf62 in the cytoplasm and is involved in actin organization and ciliogenesis within zebrafish (Bontems et al., 2014). Although the localization of TTC17 in this work shows it to be in the cytoplasm, which is contrary to our findings of TTC17 being an ER resident protein, the interaction between TTC17 and C2orf62 was determined using only a small GST-tagged purified region of TTC17 (aa 945-1041), which excludes TTC17's N-terminal ER targeting sequence. Additionally, the 
antibody used to show cellular localization (HPA038508) recognizes TTC17 in the ER in rodent cells, however, it also recognizes cytoskeletal filaments, which could explain cytoplasmic localization and actin association.

Among the novel ER-TPR containing proteins identified by an in silico search, TTC17 transcripts are the most significantly up-regulated by ER stress. Interestingly, the basal mRNA levels and fold induction under stress conditions is very similar to that of previously characterized ER TPR-containing proteins ERdj6, FICD and SEL1L. All four proteins are significantly up-regulated by calcium dysregulation, which has been shown to readily lead to ER stress. A number of ER-trafficked and resident proteins are dependent upon calcium binding for function and thus any mis-regulation not only induces total cellular signaling responses but also reduces the capacity of the ER to properly perform folding functions (Ashby and Tepikin, 2001). In addition to its transcriptional up-regulation by pharmacologically induced ER stress, endogenous TTC17 was highly up-regulated in genetically mutated cells constitutively under adaptive stress. Furthermore, the expression of TTC17 was increased when the growth temperature of these cells was increased, as was the expression of ER HSP chaperones BiP and GRP94. Although, BiP and GRP94 belong to the HSP family, neither ER localized paralogs possess heat shock elements (HSEs) within their promoters, thus are not known to be regulated by heat stress (Casas, 2017; Mahat et al., 2016). Interestingly, studies in yeast have shown that a number of ER localized proteins are up-regulated by the heat shock response under conditions of existing ER stress, thus providing a potential connection between the two stress response systems (Liu and Chang, 2008). 
As mentioned previously, the cellular HSP70s and HSP90s are highly abundant and highly regulated within cells demonstrating their importance to maintaining cellular homeostasis. A number of TPR-containing proteins have been shown to act as cochaperones of these chaperone family members, aiding in spatial organization and function for maximum efficiency (Assimon et al., 2015). ERdj6 and FICD are known TPR-containing co-chaperones of BiP. ERdj6 interacts with BiP through its J-domain and the TPRs have been shown to bind unfolded substrate (Rutkowski et al., 2007). Interestingly, ER stress reduces the efficiency of ERdj6 translocation into the ER leading to accumulation of the protein in the cytosol. This cytosolic pool functions to modulate translation in response to cellular stresses. FICD also interacts with BiP through its FIC domain and reversibly modifies BiP by AMPylation, which affects BiPs activity (Preissler et al., 2015). FICD expression is up-regulated under stress conditions, whereby it quickly de-AMPylates BiP thus creating a readily available, active pool of the chaperone when misfolded or unfolded proteins have accumulated. It is not yet understood what FICD's two TPR motifs interact with and how they contribute to FICD's function. Both FICD and ERdj6 aid to modulate BiPs activity under changing cellular conditions. Although $\mathrm{BiP}$ is actively transcribed upon the induction of the UPR, enhancing the activity of the $\mathrm{BiP}$ protein that is already present within the ER is an efficient method of rapidly attenuating folding stress.

One of the most well characterized TPR-containing co-chaperones is HOP, the Hsp70/Hsp90 Organizing Protein, which acts as a bridging chaperone binding both cytosolic HSP90 and HSP70 for efficient client transfer between chaperone systems (Scheufler et al., 2000). HOP spatially coordinates the two chaperones by interacting with 
their C-terminal tails. An ER bridging co-chaperone has yet to be identified, but TTC17 may be a candidate. HOP possesses three clusters of three TPR motifs each, one of which interacts with the cytosolic HSP70 (TPR1) and another TPR cluster (TPR2A) interacts with HSP90. All TPR clusters in HOP recognize the four C-terminal residues of HSP70/90, EEVD, and bind via carboxylate clamp. Other TPR-containing proteins have also been shown to interact with HSP70/90 in a similar manner using clusters of three and four consecutive TPRs to bind the C-terminal EEVD chaperone motif, such as TOM70 and SGTA (Roberts et al., 2015; Young et al., 2003). Chaperones of the ER possess a C-terminal retention sequence, Lys-Asp-Glu-Leu (KDEL), that could potentially act as a ligand for a co-chaperone like TTC17.

TTC17 possesses two predicted clusters of three and four sequential TPR motifs, respectively. Predictive modeling and electrostatic mapping of these two clusters show that they both possess a patch of positive residues lining the concave surface and could therefore attract negatively charged residues, like the Asp and Glu (DE) in KDEL (Figure 3.7). Analysis of the shot-gun proteomics results showed that many of the chaperones ( $40 \%$ ) that putatively interact with TTC17 possess a C-terminal KDEL, or similar charge sequence (i.e. HDEL, RDEL, HDEF, HEEF, and REEL) (Figure 3.6C). Surprisingly, only 18 proteins within the human proteome contain a C-terminal KDEL and all are ER resident. Since there are far more ER resident proteins than 18 , it is possible that the other proteins might possess a similar charge sequence and be retained by KDEL receptors or sequestered in the ER by another protein, possibly TTC17 (Bräuer et al., 2019). Additionally, it is possible that TTC17 interacts with a short sequence on ER chaperones via its TPRs under stress conditions, modulating their activity dependent on cellular 
homeostasis cues. Further studies are necessary to better understand what part of TTC17

is responsible for interacting with chaperones and under what cellular conditions do these interactions take place.

\subsection{Materials and Experimental Procedures}

\subsubsection{Plasmids and reagents}

Dulbecco's modified Eagle's medium (DMEM), $\alpha$ MEM GlutaMax, fetal bovine serum, penicillin and streptomycin were purchased from Invitrogen/ThermoFisher Scientific. Easy-Tag [35S]-Cys/Met was purchased from PerkinElmer Life Sciences. Sprotein-agarose beads and S-tag antibody were purchased from EMD Millipore. Endo H, PNGase F, Protoscript II first strand cDNA synthesis kit and all cloning reagents were purchased from New England Biolabs. FastStart SYBR Green qPCR mix was purchased from Roche Diagnostics, and all primers were acquired from IDT DNA. IRDye® $800 \mathrm{CW}$ Goat anti-Mouse IgG, IRDye® 680RD Goat anti-Rabbit IgG and IRDye® 680RD Donkey anti-Goat IgG were purchased from Li-COR Biosciences. Antibodies directed toward the following antigens were also purchased: calnexin (Enzo Life Sciences); Glucosidase II $\alpha$ (ThermoFisher Scientific); GRP94 (ThermoFisher Scientific); TTC17 (SCBT); BiP (gift from Dr. Linda Hendershot), GRP170 (gift from Dr. Linda Hendershot); $\beta$-tubulin (Cell Signaling Technologies); GM130 (BD Biosciences) and KDEL (Enzo Life Sciences). TTC17 cDNA was created by isolating RNA (HEK293T cells) and cloned into pcDNA3.1 A-, a plasmid harboring a C-terminal S-tag, using standard molecular biology techniques. All other chemicals were obtained from Sigma.

\subsubsection{Cell lines/Tissue Culture}


HEK293T, 293A or COS7 cells were grown in DMEM supplemented with $10 \%$ fetal bovine serum, $100 \mathrm{U} / \mathrm{mL}$ penicillin and $100 \mathrm{mg} / \mathrm{ml}$ streptomycin. WT CHO and MI8-5 cells were grown in $\alpha$ MEM GlutaMax supplemented with $10 \%$ fetal bovine serum and $100 \mathrm{U} / \mathrm{mL}$ penicillin and $100 \mathrm{mg} / \mathrm{ml}$ streptomycin.

\subsubsection{In silico analysis of TTC17}

The primary amino acid sequence of TTC17 was analyzed by UniProtKB and TPRpred to identify the number and position of putative TPR domains (Karpenahalli et al., 2007, 2019). Hydrophobic regions were identified by the $\Delta \mathrm{G}$ software, which predicts transmembrane domains (Hessa et al., 2007). Putative N-linked glycosylation sites were identified by NetNGlyc4.0 (Gupta, 2002).

\subsubsection{Affinity Purification and glycosylation assay}

Transfected cells were lysed in NP-40 buffer (1\% NP-40, $150 \mathrm{mM} \mathrm{NaCl}, 50 \mathrm{mM}$ Tris-HCl $[\mathrm{pH} 8.0])$. All steps were conducted at $4{ }^{\circ} \mathrm{C}$. The post-nuclear supernatant (PNS) was isolated by centrifugation followed by either direct glycosidase treatment with appropriate enzymes and buffers prior to trichloroacetic acid precipitation or pre-clearing with un-conjugated agarose beads for $1 \mathrm{hr}$. Cleared supernatant was incubated with Sprotein agarose beads overnight and subsequently washed twice with wash buffer $(0.1 \%$ NP-40, $150 \mathrm{mM} \mathrm{NaCl}, 50 \mathrm{mM}$ Tris- $\mathrm{HCl}$ [pH 8.0]). After the final wash, glycosylation assays were performed by adding appropriate buffers and either mock, Endo $\mathrm{H}$ or PNGase F enzymes according to the manufacturer's protocol. Finally, reducing sample buffer was added to all samples and they were analyzed by SDS-PAGE.

\subsubsection{Confocal Microscopy}


Cells were fixed with $3.7 \%$ paraformaldehyde in phosphate buffered saline (PBS) for 15 min followed by permeabilization with $0.1 \%$ triton $\mathrm{X}-100$ for $15 \mathrm{~min}$ at $25^{\circ} \mathrm{C}$. If cells were transfected with TTC17 S-tag cDNA, the transfection was performed approximately $16 \mathrm{hr}$ prior to fixing. Slides were stained with the indicated primary antibodies followed by staining with appropriate Alexa Fluor 488 or 594 secondary antibodies in immunostaining buffer (10\% fetal bovine serum in 1X PBS). Slides were rinsed and mounted onto cover slips with VectaShield (Vector Laboratories). Images were obtained with a Fluoview 1000 MPE, 1X81 motorized inverted research microscope (Olympus Inc.) equipped with a Hamamatsu C8484-05G camera. All images were acquired with a Plan Apo $N$ 60x 1.42NA lens and processed by using the FV10-ASW and Adobe Photoshop software.

\subsubsection{Alkaline extraction}

Alkaline extraction was performed as previously described (Sunryd et al., 2014). Briefly, cells were resuspended in ice-cold homogenization buffer (20 mM HEPES, 5 $\mathrm{mM} \mathrm{KCl}, 120 \mathrm{mM} \mathrm{NaCl}, 1 \mathrm{mM}$ EDTA, and 0.3 M sucrose [pH 7.5]) and passed through a 25 -gauge needle 20 -times. All subsequent steps were conducted at $4{ }^{\circ} \mathrm{C}$. The homogenate was centrifuged at $1,000 \mathrm{~g}$ for $10 \mathrm{~min}$ to pellet the nuclear fraction. The remaining PNS was centrifuged at 45,000 rpm in Beckman rotor (TLA 120.2) for 10 min to separate the cytosol (supernatant) from the cellular membranes (pellet). The cellular membrane fraction was resuspended in homogenization buffer, and a portion of the resuspended membranes was incubated with $0.1 \mathrm{M} \mathrm{Na} 2 \mathrm{CO}_{3}(\mathrm{pH} 11.5)$ for 30 min on ice. The alkaline extracted portion was centrifuged at $65,000 \mathrm{rpm}$ for $20 \mathrm{~min}$ through a sucrose cushion (50 mM triethanolamine, $0.3 \mathrm{M}$ sucrose [pH 7.5]) to separate soluble 
proteins from membrane proteins in the supernatant and pellet, respectively. The $\mathrm{pH}$ was adjusted in the alkaline extracted sample with $1 \mathrm{M}$ Tris- $\mathrm{HCl}(\mathrm{pH}$ 7.5). The WCL, Cyt, TM and S fractions were precipitated with $10 \%$ trichloroacetic acid prior to adding reducing sample buffer and analyzing samples via SDS-PAGE.

\subsubsection{Trypsin protection}

Transfected cells were radiolabeled for $1 \mathrm{hr}$ prior to resuspension in cold homogenization buffer (10 mM HEPES [pH 7.4], $10 \mathrm{mM} \mathrm{KCl,} 1.5 \mathrm{mM} \mathrm{MgCl}, 5 \mathrm{mM}$ sodium EDTA, $5 \mathrm{mM}$ sodium EGTA and $0.25 \mathrm{M}$ sucrose) and passed through a 25-gauge needle 20-times. All subsequent steps were conducted at $4{ }^{\circ} \mathrm{C}$. The homogenate was centrifuged at 1,000g for $10 \mathrm{~min}$ to pellet the nuclear fraction. The remaining PNS was centrifuged at 45,000 rpm in Beckman rotor (TLA 120.2) for 10 min to separate the cytosol (supernatant) from the cellular membranes (microsomes). The microsomes were resuspended in homogenization buffer containing $0.1 \mathrm{M} \mathrm{NaCl}$ and $10 \mu \mathrm{g}$ trypsin and/or

triton X-100 was added to a final concentration of $0.1 \%$. After incubation at $27{ }^{\circ} \mathrm{C}$ for 15 min, the reaction was quenched with $100 \mu \mathrm{g}$ soybean trypsin inhibitor. Reducing sample buffer was added and analyzed via SDS-PAGE.

\subsubsection{Immunoblotting and affinity purification}

Non-transfected and transfected cells were lysed in NP-40 buffer and the postnuclear supernatant (PNS) was isolated by centrifugation followed by total protein concentration determination using $595 \mathrm{~nm}$ Protein Assay Dye Reagent (Bio-Rad). All subsequent steps were conducted at $4{ }^{\circ} \mathrm{C}$. Equal amounts of total protein were isolated from all samples by precipitation with $10 \%$ trichloroacetic acid or affinity purification using S-protein agarose beads. Finally, reducing sample buffer was added to all samples 
and they were analyzed by SDS-PAGE. Proteins were transferred to a polyvinylidene difluoride membrane and immunoblotted with the appropriate antisera. Blots were developed and TIFF files were acquired using a LI-COR Odyssey CLx Imager. Densitometric quantification of western blots was performed using ImageJ software (Fiji). The amount of TTC17, BiP and GRP94 was calculated by dividing the amount of each protein with the amount of $\beta$-tubulin. Error bars represent the standard deviation for three independent experiments.

\subsection{9 qRT-PCR}

HEK293A cells were treated with regular growth media or dithiothreitol (2 mM) for $2 \mathrm{hr}$ or tunicamycin $(1 \mu \mathrm{g} / \mathrm{mL})$, thapsigargin $(3 \mu \mathrm{M})$, brefeldin $\mathrm{A}(2.5 \mu \mathrm{g} / \mathrm{mL})$ and MG132 $(2.5 \mu \mathrm{M})$ for $24 \mathrm{hr}$ prior to RNA isolation with RNAeasy Mini Kit (Qiagen). One $\mu \mathrm{g}$ of purified RNA was reverse transcribed into cDNA using the Protoscript II Reverse Transcriptase kit (New England Biolabs). Quantitative real time polymerase chain reactions (qRT-PCR) were performed in $20 \mu \mathrm{L}$ reactions using the FastStart universal SYBR Green master (Rox) kit (Roche diagnostics Corp.) on an Mx3000P real-time PCR machine (Agilent Technologies Inc.) according to manufacturer's instructions. Changes in mRNA levels were calculated using the change in cycle threshold value method with $\beta$-actin as the reference gene (Pfaffl, 2001). Statistical analysis of the data was calculated using GraphPad Prism 5.0 (GraphPad software) and significance between treatment groups was determined using unpaired T-tests.

The following primers were used: $\beta$-actin (5' GCACTCTTCCAGCCTTCC 3', 5' TGTCCACGTCACACTTCATG 3’), TTC17 (5’ ACCAAGCAAACCTAGAGATCAC 
3', 5' GTACTCACGATGGCAGTCAG 3'), SEL1L (5'

GAGGCCATATCTTGGCTTTCT 3', 5' GGCCTCGTTCACATACATTCT 3') FICD

(5' TATGGTGTCTGCTGTGTCTTG, 5' CTCCTCCACCTTAGTGCAAAT 3'), ERdj6

(5' CTTTATGCTGTCTGGAGGGAAG 3', 5' GCAAGGCTGTGAAGAGAAGA 3').

\subsubsection{Shotgun LC-MS/MS and silver stain}

HEK293T cells were transfected as indicated and isolated by isotonic

fractionation and ultracentrifugation to purify microsomes. These microsomes were then resuspended with $1 \% \mathrm{NP}-40$ detergent buffer followed by affinity purification with Sprotein agarose beads. Affinity purifications (APs) were washed twice with wash buffer containing $0.1 \% \mathrm{NP}-40$ and twice in $50 \mathrm{mM}$ ammonium carbonate before adding reducing sample buffer. A portion of the sample was loaded onto a SDS-PAGE and subjected to silver staining to verify the enrichment of putative binding partners (Chevallet et. al Nature Protocols 2006). Once appropriate enrichment had been observed, a portion of the AP was loaded onto a SDS-PAGE gel (10-40\% bis-tris protein gel NuPage), and the dye front was allowed to migrate $\sim 1 \mathrm{~cm}$ into the resolving gel. The sample containing area of the gel was excised and prepared for in-gel trypsin digestion. Tryptic peptides generated by trypsinization were loaded on a LC-MS/MS instrument followed by protein identification. Proteins were scored based on the number of unique peptides that were identified from the corresponding protein and averaged across three experiments. 
A

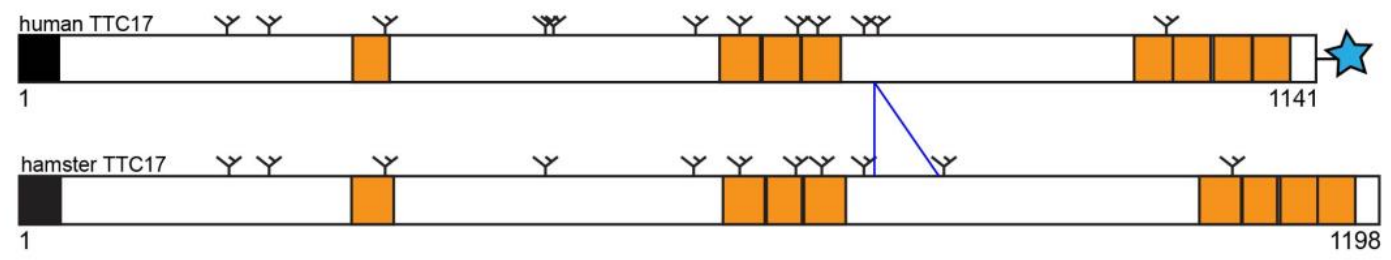

Signal Sequence $\square$ TPR Motif Y Predicted N-Glycosylation site

B

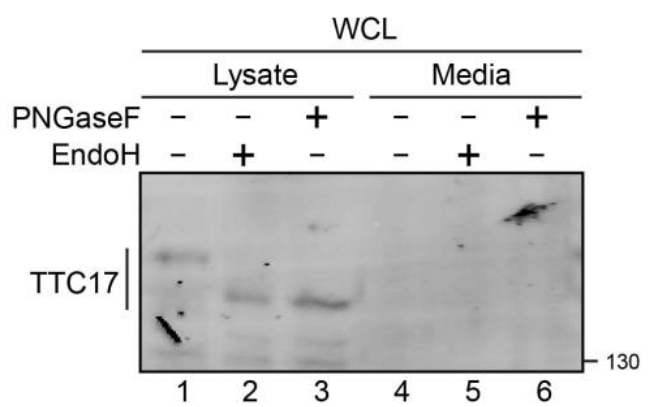

D
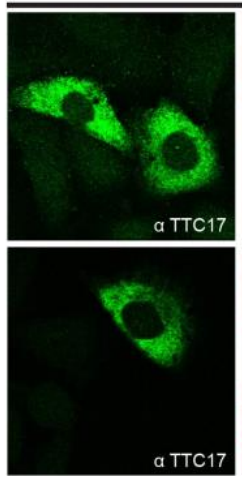

TTC17
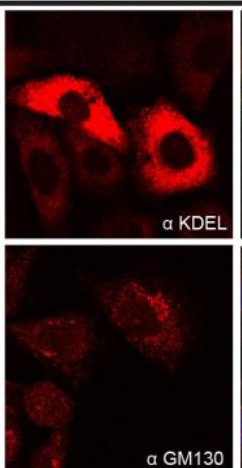

C

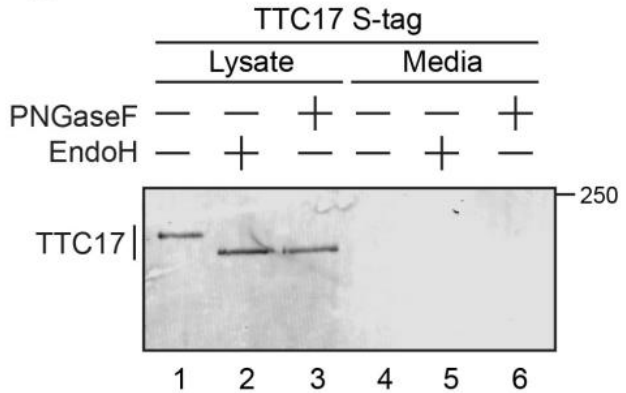

$\mathrm{E}$
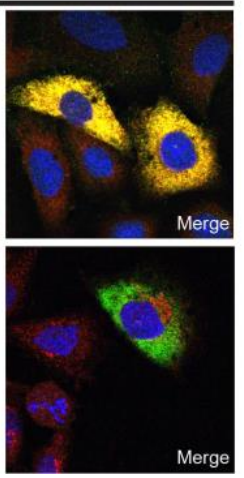

TTC17 S-tag
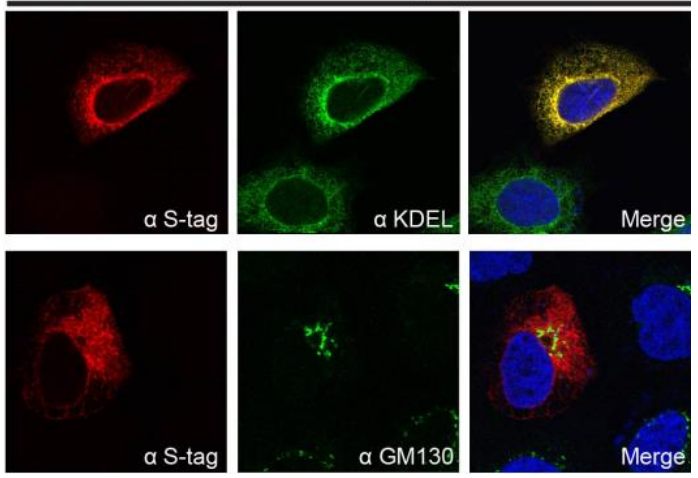

Figure 3.1. TTC17 is an ER resident TPR-containing protein. (A) Our screen for novel secretory adapter proteins identified TTC17 as a putative TPR-containing protein that was potentially targeted to the ER by an N-terminal signal sequence (black square). The position of tetratricopeptide repeats (TPRs) (orange squares) is designated and predicted $\mathrm{N}$-linked glycosylation sites are indicated by small, black, branched structures. Human TTC17 cDNA was C-terminally S-tagged (blue star) for biochemical studies. The hamster and human TTC17 sequences are designated and differ by fifty amino acids as indicated by the angled blue lines. (B) WT CHO cell lysate and media was collected and subjected to a glycosylation assay with either EndoH (lanes 2 and 5) or PNGaseF (lanes 3 and 6) digestion as indicated. Reducing sample buffer was added and the samples were analyzed by $7 \%$ SDS-PAGE prior to detection by immunoblotting with antisera directed 
against endogenous TTC17. (C) HEK293T cells were transfected with S-tagged TTC17 and TTC17 was affinity purified from the cell lysate and media using S-protein agarose. Samples were then subjected to a glycosylation assay with either EndoH (lanes 2 and 5) or PNGaseF (lanes 3 and 6) digestion as indicated. Reducing sample buffer was added and the samples were analyzed by a $6 \%$ SDS-PAGE and immunoblotted for the S-tag epitope. (D) Cellular localization of endogenous TTC17 was investigated by confocal microscopy. WT CHO cells were fixed cells and stained with TTC17, KDEL (ER) or GM130 (Golgi) antisera. Nuclei were visualized by DAPI staining (blue). (E) COS7 cells were transfected with human TTC17 cDNA. Fixed cells were stained with S-tag, KDEL (ER) or GM130 (Golgi) antisera. Nuclei were visualized by DAPI staining (blue). 
A

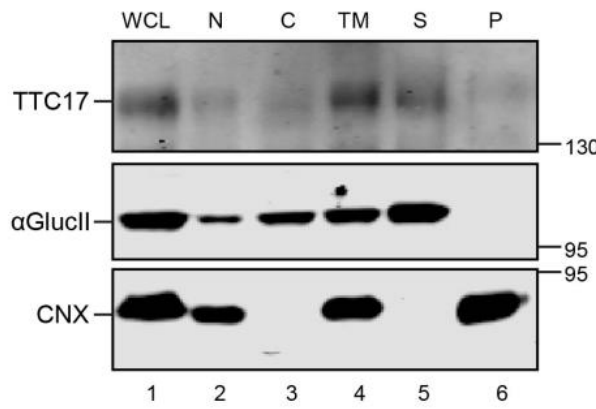

B
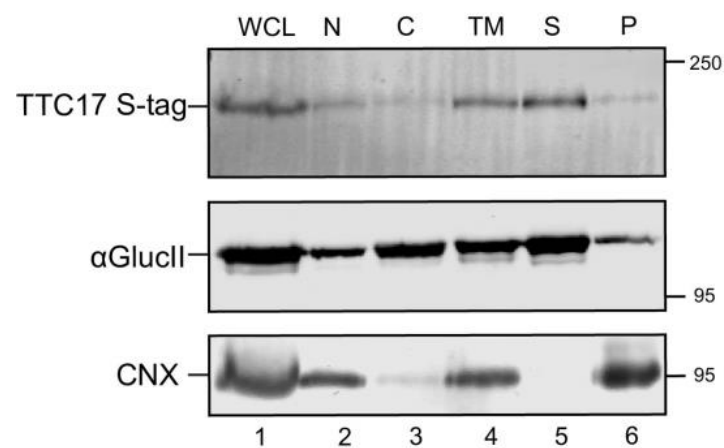

C

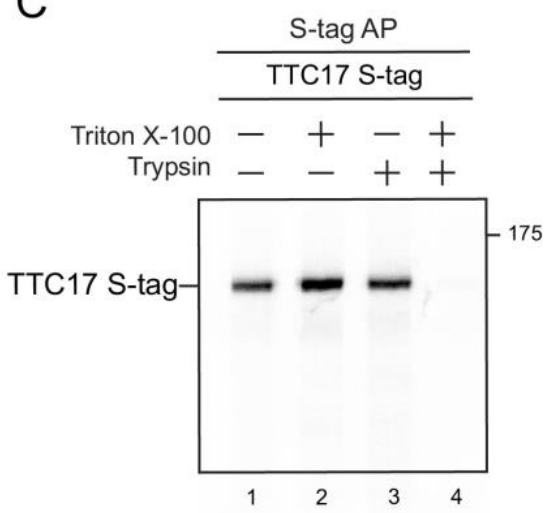

Figure 3.2. TTC17 is a soluble protein that resides within the ER lumen. (A) WT CHO cells were homogenized and fractionated prior to alkaline extraction. The fractions collected were whole cell lysate (WCL), nucleus (N), cytosol (C), total membrane (TM), as well as supernatant $(\mathrm{S})$ and pellet $(\mathrm{P})$ fractions upon alkaline extraction of the TM. The WCL, C, and S fractions were then precipitated with trichloroacetic acid, and all samples were resuspended in sample buffer prior to the proteins being detected by immunoblotting with antisera directed against TTC17, calnexin (CNX) and Glucosidase II $\alpha$ ( $\alpha$ GlucII). (B) HEK293T cells transfected with S-tagged TTC17, homogenized, fractionated prior to alkaline extraction and samples collected as described in A. Samples were analyzed via 7\% SDS-PAGE prior to immunoblotting with antisera against the Stag epitope, calnexin (CNX) and Glucosidase II $\alpha(\alpha$ GlucII). (C) TTC17-S-tag was expressed in HEK293T cells. After radiolabeling with [35S]-Cys/Met for $1 \mathrm{hr}$, cells were homogenized, and microsomes were purified by ultracentrifugation then resuspended in homogenization buffer. Aliquots of the ER microsomes were incubated for $10 \mathrm{~min}$ at 27 ${ }^{\circ} \mathrm{C}$ with or without triton X-100 and trypsin as indicated. Samples were subsequently subjected to affinity purification using S-protein agarose beads. Samples were resolved on a reducing 9\% SDS-PAGE. 


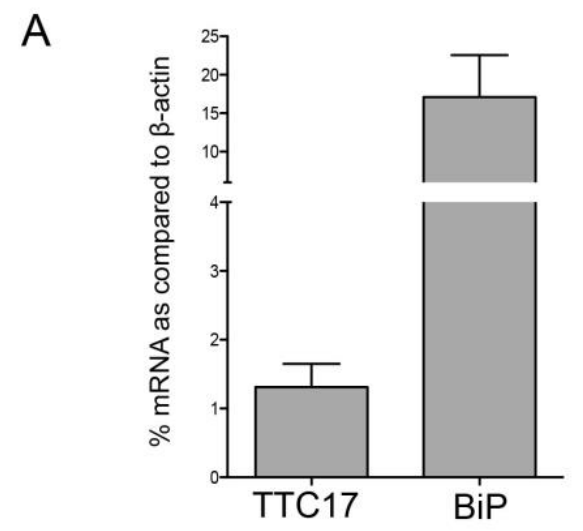

B

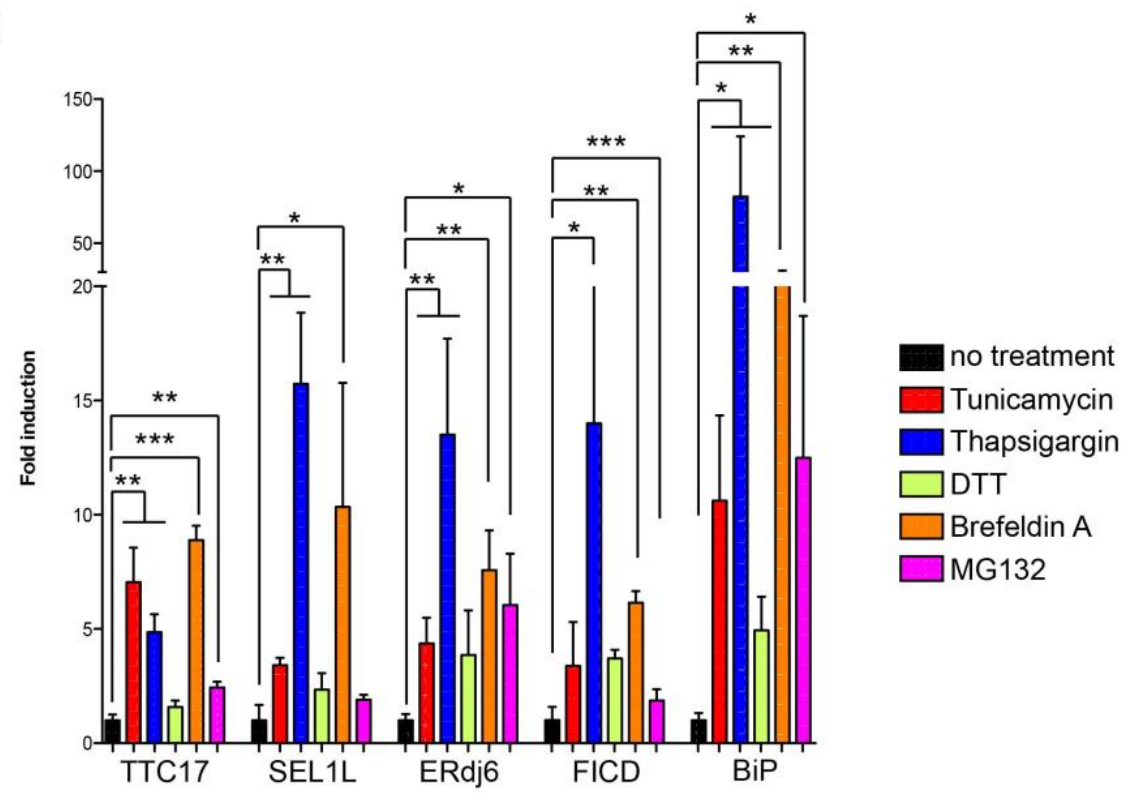

Figure 3.3. Basal transcript abundance and fold induction of TTC17 by ER stress. (A) RNA from HEK293A cells, grown under normal conditions, was harvested. RNA was reverse transcribed to cDNA followed by qRT-PCR with appropriate primers. Basal mRNA abundance was assessed using $\beta$-actin as a reference. Error bars represent standard deviation from at least three independent experiments. (B) HEK293A cells were treated with regular growth media or with $2 \mathrm{mM}$ DTT for $2 \mathrm{hr}, 1 \mu \mathrm{g} / \mathrm{mL}$ tunicamycin, 3 $\mu \mathrm{M}$ thapsigargin, $2.5 \mu \mathrm{g} / \mathrm{mL}$ brefeldin A or $2.5 \mu \mathrm{M}$ MG132 for $24 \mathrm{hr}$ prior to RNA purification. RNA was reverse transcribed to cDNA followed by qRT-PCR with appropriate primers, and changes in gene expression were calculated using $\beta$-actin as a reference. Statistical significance between treatment groups was determined using an unpaired T test. *, ** and *** indicates a P-value of less than $0.05,0.01$ and 0.001 , respectively. Error bars represent standard deviation from at least three independent experiments. 

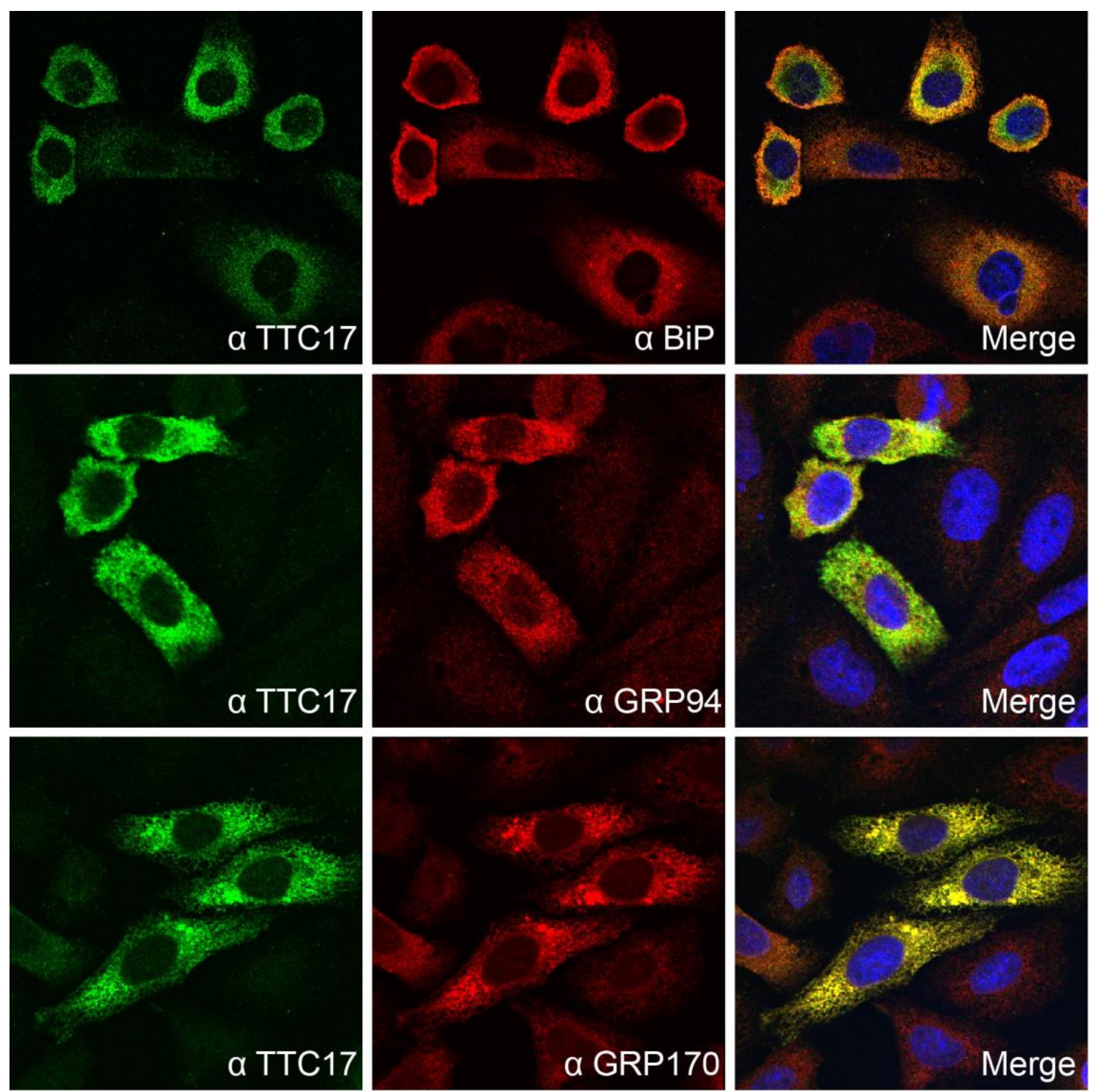

Figure 3.4. TTC17 and ER stress chaperones show elevated expression in cells. WT CHO cells were fixed and stained with TTC17, BiP, GRP94 and GRP170 antisera. Nuclei were visualized by DAPI staining (blue). 


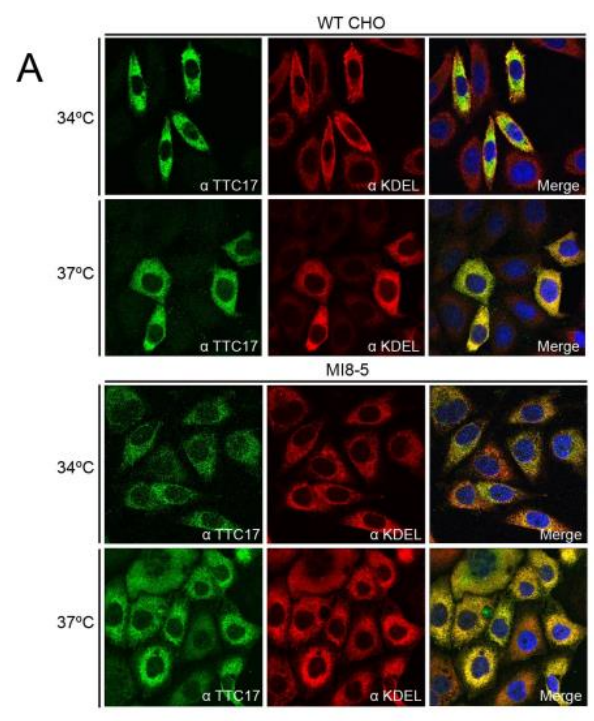

B
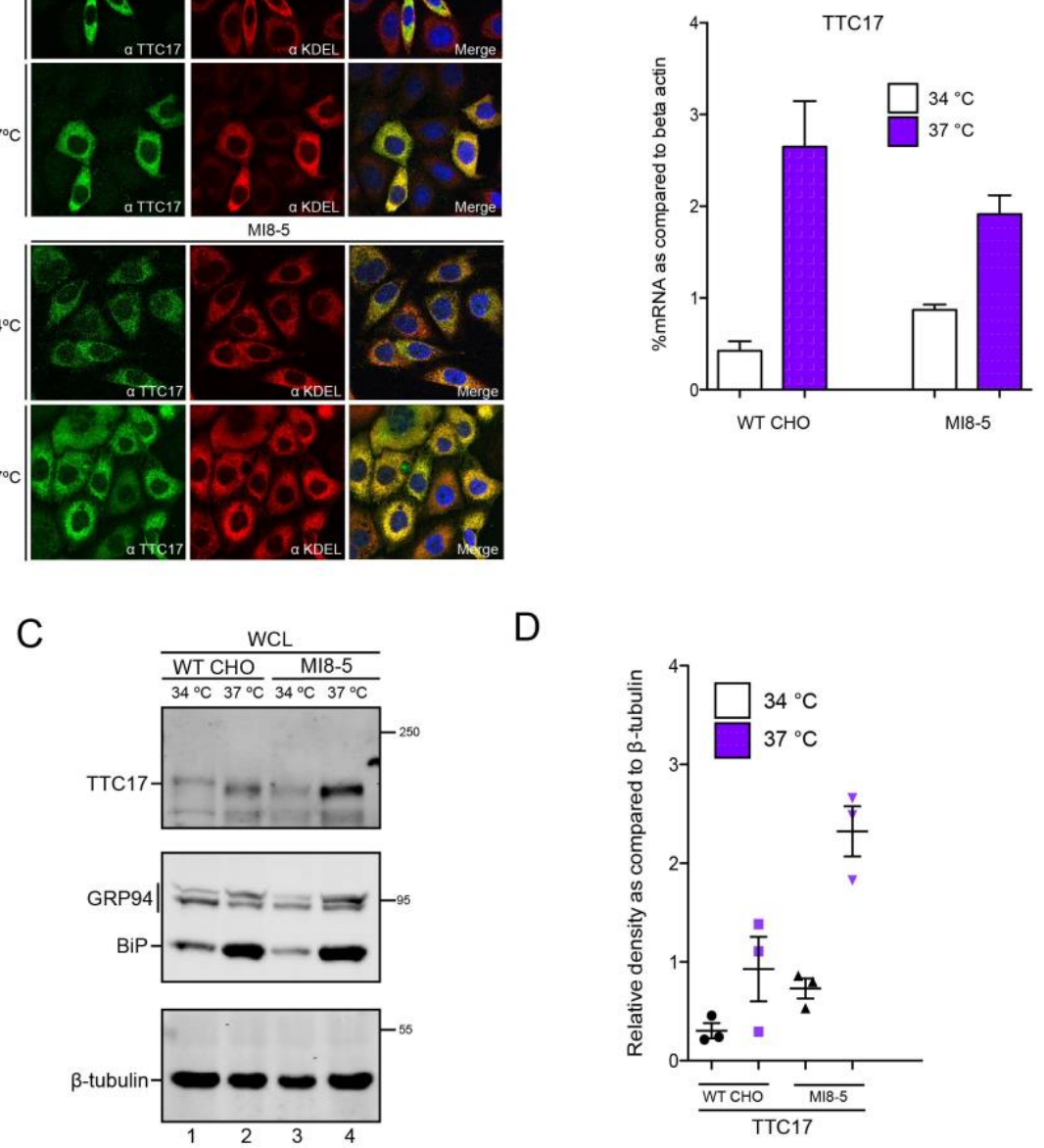

Figure 3.5. TTC17 is up-regulated by heat in reglucosylation deficient cells. (A) WT CHO and MI8-5 cells were fixed and stained with TTC17 and KDEL (ER) antisera. Nuclei were visualized by DAPI staining (blue). (B) RNA from WT CHO and MI8-5 cells, grown under normal conditions at the temperatures indicated, was harvested. RNA was reverse transcribed to cDNA followed by qRT-PCR with appropriate primers. Basal mRNA abundance was assessed using $\beta$-actin as a reference. Error bars represent standard deviation from at least three independent experiments. (C) WT CHO and MI8-5 cell lysates grown at $34^{\circ} \mathrm{C}$ and $37^{\circ} \mathrm{C}$, respectively, were collected. Samples were run on a $6 \%$ SDS-PAGE prior to detection by immunoblotting with antisera directed against endogenous TTC17, KDEL and $\beta$-tubulin. (D) Quantification of relative density of TTC17 from WT CHO and MI8-5 cells grown at indicated temperatures, respectively. Error bars represent standard deviation. 
A

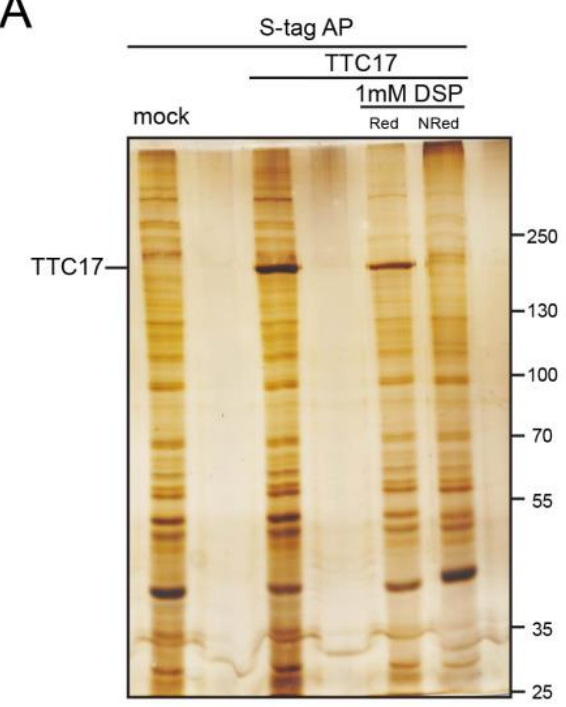

B
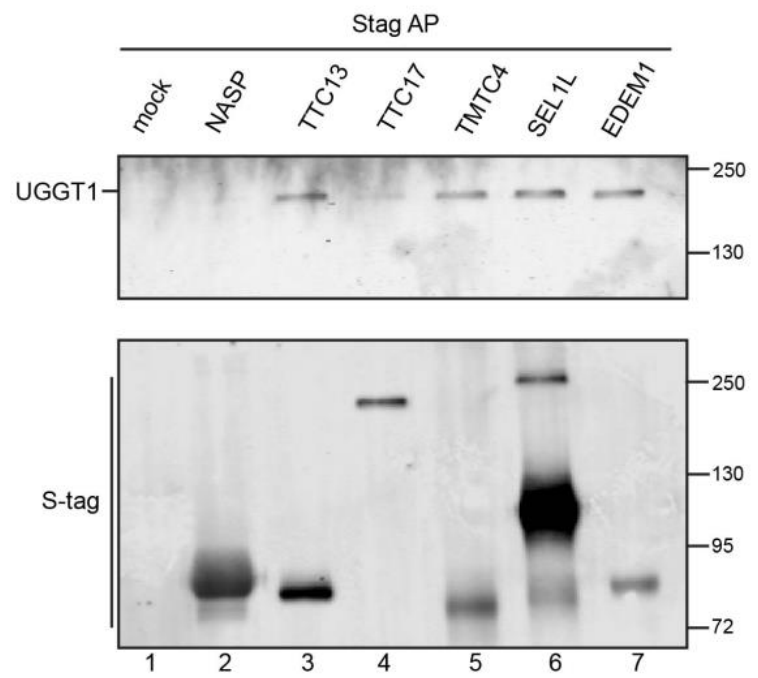

C

\begin{tabular}{|lccc|c}
\hline Common Name & Mock & Unique Peptides & Corrected & \\
\cline { 1 - 3 } GRP94 & 17 & 49 & 32 & KDEL \\
TTC13 & 0 & 31 & 31 & LKKL \\
Ribophorin I & 0 & 25 & 25 & LDAL \\
Glucosidase Ila & 7 & 19 & 13 & IHLR \\
p180 & 10 & 22 & 12 & GTSV \\
ERp57 & 10 & 21 & 11 & QEDL \\
Calretiulin & 0 & 18 & 18 & KDEL \\
COPI Vesicle Coat & 0 & 10 & 10 & YEIL \\
UGGT1 & 0 & 10 & 10 & REEL \\
PDI & 8 & 17 & 10 & KDEL \\
ERdj6 & 3 & 12 & 9 & FHFN \\
BiP & 27 & 34 & 7 & KDEL \\
Serpin H1 & 5 & 11 & 7 & RDEL \\
Ribophorin II & 0 & 5 & 5 & RTAH \\
Calumenin & 0 & 4 & 4 & HDEF \\
STT3A & 0 & 4 & 4 & LSRT \\
Calnexin & 9 & 12 & 3 & PRRE \\
Sec23A & 2 & 5 & 3 & SSAA \\
AP-1 complex subunit gamma-1 & 0 & 3 & 3 & QSWQ \\
45 kDa calcium-binding protein & 0 & 3 & 3 & HEEF \\
beta-COPI & 6 & 9 & 3 & ILDD
\end{tabular}

Figure 3.6. ER localized S-tagged TTC17 interacts with a number of chaperones. (A) A shotgun liquid chromatography-mass spectrometry/mass spectrometry (LC-MS/MS) approach was used to identify potential binding partners of TTC17. HEK293T cells were transfected with TTC17-Stag plasmid followed by isotonic fractionation and ultracentrifugation to purify microsomes. The microsomes were resuspended in a buffer with detergent, and the tagged protein was affinity purified with S-protein agarose beads. The affinity purified complex was run on a short SDS-PAGE prior to in-gel trypsin digestion and silver staining. The peptides generated by trypsinization were loaded onto a LC-MS/MS instrument followed by peptide identification. Proteins were scored based on the number of unique peptides that were identified. (B) HEK293T cells were transfected 
with S-tagged cDNA of NASP, TTC13, TTC17, TMTC4, SEL1L and EDEM1. Cells and media were collected, and S-tagged proteins were affinity purified using S-protein agarose. Samples were then subjected to immunoblotting with antisera directed against the S-tag epitope and endogenous UGGT1. (C) Potential binding partners identified from shot-gun proteomics approach described in (A). The unique number of peptides was determined by averaging three independent experiments and then subsequently compared to mock. The corrected column represents the difference and is displayed in ascending order of number of peptides. The four $\mathrm{C}$-terminal residues for each protein is displayed to the right of each row. 

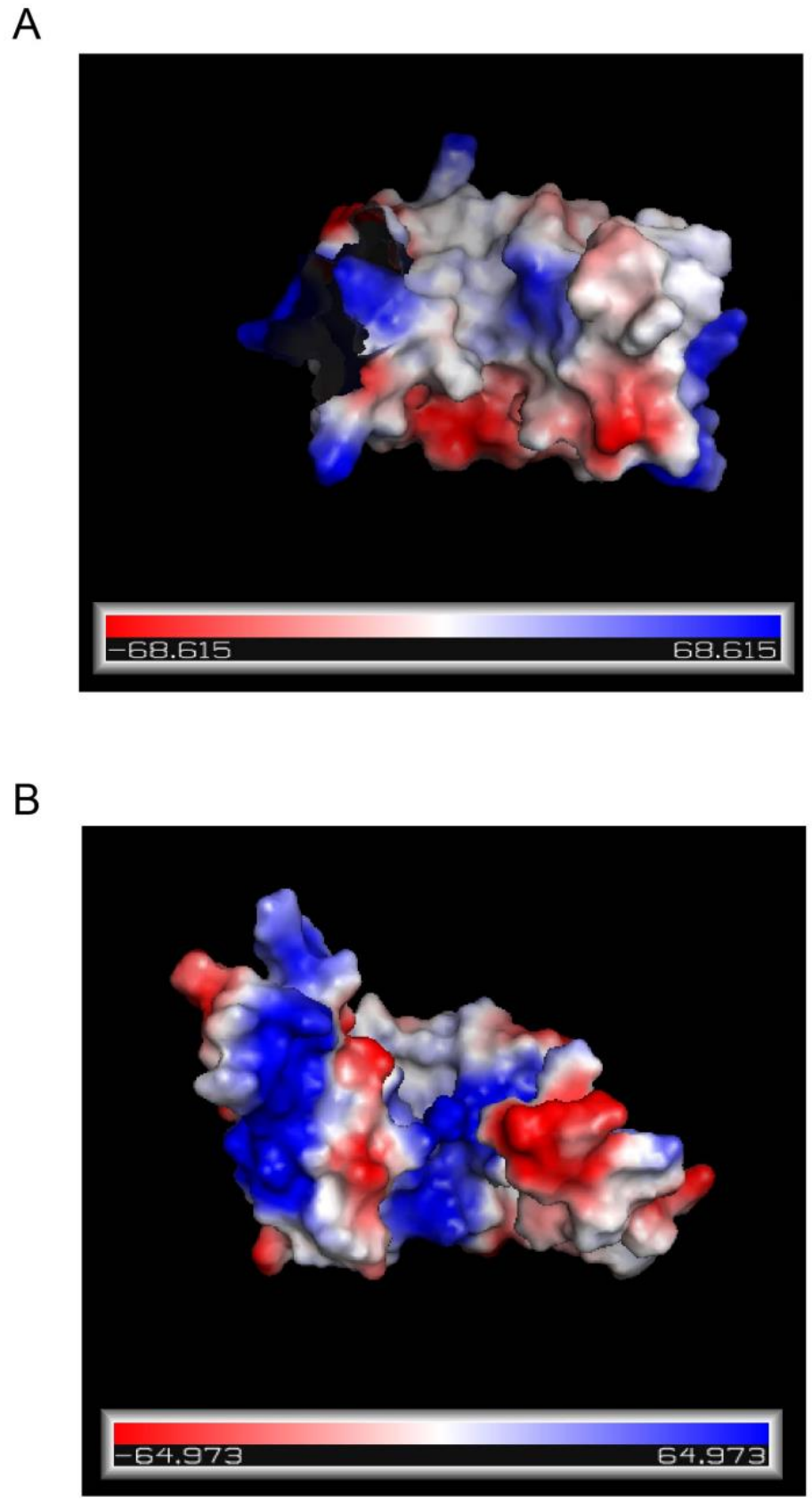

Figure 3.7. Structural models of TTC17 TPR clusters reveal positively charged patches on the concave surface. (A) Surface model (Phyre 2.0) of the first cluster of TPRs (2-4) in TTC17 depicting negatively charged residues (red) and positively charged residues (blue). (B) Surface model (Phyre 2.0) of the second, C-terminal cluster of TPRs (5-8) in TTC17 depicting charged residues as described in A. 


\section{CHAPTER 4}

\section{CONCLUSIONS AND FUTURE DIRECTIONS}

A bioinformatic screen for novel TPR-containing proteins of the endoplasmic reticulum (ER) resulted in a deeper understanding of how TPR-containing proteins contribute to protein homeostasis. The ER is a highly dynamic, multi-functional organelle responsible for several essential cellular processes. One third of the mammalian proteome is targeted to the ER for specialized folding and the ER possesses numerous factors that aid in this process, including TPR-containing proteins involved in protein translocation, co-chaperone function and protein modification. Approximately $40 \%$ of cellular TPRcontaining proteins have been suggested to contribute to maintaining the human proteostasis network and the TPR-containing proteins identified and characterized in this study contribute to protein homeostasis within the ER (Brehme et al., 2014; Graham et al., 2019).

\subsection{TMTC3 is a novel O-mannosyltransferase}

TMTC3 was found to O-mannosylate E-cadherin and be a member of a novel class of putative O-mannosyltransferases that modify the cadherin superfamily of proteins (Figure 2.5) (Larsen et al., 2017a). Studies on yeast protein Omannosyltransferases (Pmt1-7) and previously characterized mammalian POMT1 and 2 showed that they form distinct complexes amongst each other and this complex formation is required for full mannosyltransferase activity (Gentzsch et al., 1995; Girrbach et al., 2000). While the nature of the TMTC1-4 complexes is not known, sucrose gradient and co-affinity purification data shows that they interact with one another or have shared binding partners. TMTC1, TMTC2 and TMTC4 have been shown to form higher 
molecular weight complexes and interact with SERCA2B (Li et al., 2018; Sunryd et al., 2014). TMTC2 and TMTC4 were also shown to interact with calnexin (Sunryd and Johan, 2014). Additionally, shot-gun proteomic analysis showed that TMTC3 may interact with both TMTC1 and TMTC2 as four and three unique TMTC3 peptides were identified from the affinity purified TMTC1 and TMTC2 samples, respectively (Sunryd et al., 2014).

Preliminary sucrose gradient data overexpressing C-terminally S-tagged TMTC3 and TMTC4 cDNA in HEK293T cells show that they may reside in higher molecular weight complexes (Figure 4.1A). TMTC3, while very faint, appears to be in a complex of approximately four times its size and TMTC4 appears to be in a complex of double its size indicating that they could exist in hetero- or homo-oligomers to form active Omannosyltransferases. Thus far, analysis has used overexpression systems to study these proteins, therefore, further studies are required to better understand how the endogenous TMTC family members interact with one another and what cellular role each plays. To circumvent the short-comings of overexpression, we could generate antibodies against the endogenous proteins or use the CRISPR system to endogenously tag TMTC1-4, individually (Lackner et al., 2015). This approach would better preserve the stoichiometry of the TMTC complexes allowing us to verify interactions with other proteins and potential sub-regional localization within the ER. It would also allow affinity purification of endogenous complexes for structural studies.

Recently, affinity purification of FLAG-tagged Pmt1 revealed the first structure of an O-mannosyltransferase complex. The cryo-EM structure of the Saccharomyces cerevisiae Pmt1-Pmt2 complex shows that each subunit contains 11 transmembrane 
helices and a luminal $\beta$-trefoil fold that is the Mannosyltransferase, Inositol 1,4,5trisphosphate receptor and Ryanodine receptor (MIR) domain (Bai et al., 2019). The structure also reveals the substrate recognition model and catalytic site. TMTC 3 and TMTC4 possess ten and twelve putative transmembrane segments, respectively (Figure 2.1A). While we know that these proteins are integral membrane proteins and their $\mathrm{C}$ terminal TPR domains face the ER lumen, additional studies are necessary to fully understand their topology and how they might coordinate O-mannosyltransferase activity and substrate binding (Graham et. al submitted 2019). The Pmt1-Pmt2 complex showed that the two proteins interact via cytoplasmic loops and the transmembrane segments form a sizeable rhombic cavity that is proposed to allow the membrane-embedded donor substrate, Dol-P-Man, to diffuse into the catalytic site of the complex (Bai et al., 2019). It is possible that the TMTCs could form similar cavities, however structural studies are required to determine this and we have initiated studies with the researchers of the Pmt12 cryo EM studies to solve the structure of TMTC3.

In vitro $[3 \mathrm{H}]$ mannose labelling studies in yeast indicated that a diacidic (Asp-Glu) motif located in an N-terminal luminal loop is the active site of the Pmt Omannosyltransferases (Lommel et al., 2011). Interestingly, all four TMTC proteins contain putative active residues located in an $\mathrm{N}$-terminal loop. The recent structural study showed a small peptide substrate (PYTV) in complex with Pmt1-Pmt2 with the first residue in the diacidic motif (Asp77 in Pmt1 and Asp92 in Pmt2) ideally positioned to activate the hydroxyl of the acceptor threonine and the second residue (Glu78 in Pmt1 and Glu93 in Pmt2) essential for forming a salt bridge with an invariant Lys in the first loop (Bai et al., 2019). Although it appears that all four TMTC proteins possess putative 
diacidic motifs within the first $\mathrm{N}$-terminal loop, hydrophobic analysis combined with trypsin protection assays demonstrate that only TMTC 3 and TMTC4 may have catalytic residues facing the ER lumen. Critical to understanding this structure function relationship is determining the orientation of each of the hydrophobic segments within these proteins. Structural analysis would be most informative, however cellular studies could be simultaneously performed as purifying multi-pass transmembrane proteins may be challenging. Using HEK293 TMTC1/2/3/4--, we can individually express the TMTCs, isolate ER-derived microsomes and perform $[3 \mathrm{H}]$-mannose labelling on previously described peptides to determine which TMTCs possess O-mannosylation activity (Lommel et al., 2011). We can then mutate residues within the TMTCs and perform the same assay to determine the putative catalytic sites.

As mentioned previously, the TMTCs were found to O-mannosylate the cadherin family of proteins (Larsen et al., 2017a). It has recently been shown that the transmembrane region of protein O-mannosyltransferases coordinates the transfer of the Dol-P-Man to the donor substrate, however we do not know how the substrates are recruited to the active site. Based on studies on the MIR domain-containing protein, Stromal-derived factor 2 (SDF2), it has been suggested that the MIR domains present within the Pmts/POMTs are responsible for binding substrate and/or chaperones (Fujimori et al., 2017). As TPRs are protein-protein interaction motifs, we hypothesize that the C-terminal TPR domains of the TMTCs could interact with the repeat extracellular cadherin (EC) domains on cadherins to recruit them to the active site for Omannosylation (Figure 4.2B). The ARM repeat domains of $\beta$-catenin have been shown to interact with the backbone of murine E-cadherin at sites that are conserved among other 
cadherin family members (Huber and Weis, 2001). The TPR motifs of OGT contain conserved Asn like the ARM repeat domains of $\beta$-catenin that are responsible for the interaction with E-cadherin (Jínek et al., 2004). Similarly, TMTC3 and TMTC4 have a number of conserved Asn at positions 6 and 9 of their respective TPR consensus sequences.

First, we would determine if the two classes of proteins, TMTCs and cadherins, interact. Previously performed affinity purifications and subsequent proteomic analysis of the TMTC proteins did not reveal interactions with cadherins, however, TPR-containing proteins often display transient interactions as scaffold proteins. In order to circumvent this issue, we can use TurboID in living cells to efficiently label interacting partners of the TMTCs by proximity (Branon et al., 2018). This system targets the protein of choice tagged with a biotin ligase to intracellular organelles using signal sequences to label endogenous proteins that come within a few nanometers of the bait protein. We can design both full-length and TPR domain rich constructs for the each of the TMTCs to identify interactors. If the TMTCs interact with cadherins, we can mutate the conserved Asn and perform the same assay to determine whether these residues are responsible for recruitment of substrate. Alternatively, we could endogenously CRISPR tag the TMTC proteins and perform biochemical analysis with intact complexes (Lackner et al., 2015).

Glycosylation plays crucial roles in numerous cellular processes including cellcell recognition, signal transduction and ER protein quality control (ERQC) (D'Alessio et al., 2010; Haines and Irvine, 2003; Hart et al., 2007; Helenius and Aebi, 2004; Janik et al., 2010; Rambaruth and Dwek, 2011). Knockdown of the TMTCs results in a significant loss of O-mamnosylation of the cadherin family of proteins (Larsen et al., 
2017a). Cadherins are transmembrane proteins that mediate cell-cell adhesion, regulating cell contact formation and stability (Maître and Heisenberg, 2013). We found that Omannosylation of E-cadherin was primarily performed by TMTC3 and it affects cellular adherence through E-cadherin. Additionally, TMTC3 was the only TMTC that does not interact with SERCA2B, therefore, the TMTCs may play different cellular roles. In yeast, the different Pmt complexes exhibit specificity toward their protein substrates (Gentzsch and Tanner, 1997; Proszynski et al., 2004; Sanders et al., 1999). It is possible that the TMTC proteins O-mannosylate different classes of substrates and their subsequent $\mathrm{O}$ mannosylation affects the substrates differently. Recently, O-mannosylation has been implicated in ERQC by tagging slow folding substrates for proteasomal degradation $\mathrm{Xu}$ et al., 2013). TMTC3 may affect the cell adhesion function of cadherins, while the other TMTCs may monitor folding of the cadherins and target them for degradation.

To this end, we have acquired slow and fast folding GFP constructs that localize to the ER. Slow ER-GFP is demonstrated to be O-mannosylated and subsequently degraded in contrast to fast folding ER-GFP (Xu et al., 2013). Using HEK293 TMTC1/2/3/4-- cells, we can express these constructs in the presence of individual TMTCs and monitor both modification of the "slow" unfolded ER-GFP and its rate of degradation. Additionally, we can assess O-mannosylation of classic ERAD substrates as they accumulate in the ER with proteasomal inhibition in the presence of TMTCs. If Omannosylation by the TMTCs does not act to target proteins for degradation, it may be that they modify different substrates or different parts of the substrates affecting stability, localization or function of various secretory and membrane proteins (Lommel and Strahl, 2009; Petkova et al., 2012; Yang et al., 2009). 
Since TMTC1, TMTC2 and TMTC4 have been found to interact with SERCA2B and regulate calcium dynamics, it is possible that they affect cadherin function through SERCA2B (Suisse and Treisman, 2019). Cellular calcium plays three major roles in cadherin mediated adherence. Calcium helps to rigidify the individual extracellular cadherin (EC) domains, define the structure of the dimer interface surfaces and opening of the A strand for strand swapping (Brasch et al., 2012). TMTC1-4 were found to be involved in the O-mannosylation of a number of ER resident proteins, including ERp57 (Larsen et al., 2017a). It is possible that TMTC1, 2 and 4 affect calcium dynamics of SERCA2B by O-mannosylating ERp57 (Li and Camacho, 2004). To this end, we could assess calcium dynamics in HEK293 TMTC1/2/3/4-/- cells and compare it to WT HEK293 cells as well as samples that expressed the TMTCs individually (Sunryd et al., 2014).

Protein O-mannosylation is an essential protein modification in fungi and animals (Beltrán-Valero de Bernabé et al., 2002; Gentzsch and Tanner, 1996; Immervoll et al., 1995; Mouyna et al., 2010; Willer et al., 2004, 2005). Defects in glycosylation have been found to be associated with human diseases. Mutations in TMTC3 have been shown to result in mild forms of Cobblestone Lissenceaphaly and Periventricular Nodular Heterotopia (PVNH) (Farhan et al., 2017; Jerber et al., 2016). Of the mutations we characterized, we find that three of the eight exhibit reduced stability in cells. While all are ER localized, it would be of interest to determine the mechanism of disease for the remaining five mutations. Biochemical analysis studies will include topology assessment, interacting partner analysis and in cell activity, and the ability to O-mannosylate Ecadherin. In conclusion, we have identified TMTC3 as a novel O-mannosyltransferase of 
E-cadherin, however, further structural and biochemical analysis is necessary to understand its activity and disease phenotypes.

\subsection{TTC17 is a novel ER adaptor involved in ER stress}

TTC17 was identified as a novel TPR-containing protein resident to the ER. TTC17 transcripts were up-regulated significantly by a number of ER stress inducing reagents including $\mathrm{N}$-glycan synthesis inhibition (tunicamycin), calcium depletion (thapsigargin), redox stress (dithiotreitol, DTT), inhibition of anterograde protein trafficking (brefeldin A) or proteasomal inhibition (MG132). Several essential ER chaperones are transcriptionally up-regulated by the UPR (Shinjo et al., 2013). ER stress activates stress sensors ATF6, IRE1 and PERK, representing the three branches of the UPR (Walter and Ron, 2011). Activation of each sensor produces a transcription factor that activates genes to increase folding capacity, ATF6, XBP1 and ATF4 respectively. These transcriptions factors, predominantly XBP-1, recognizes a sequence in the stress response genes defined as the ER stress response element (ERSE) (Roy and Lee, 1999). Preliminary analysis of the upstream region for the TTC17 transcript by promoter binding algorithms shows that it does not possess an ERSE.

Interestingly, studies in yeast found that UPR-deficient cells are intolerant of ER stress and the heat shock response (HSR) can rescue ER stress in vivo (Liu and Chang, 2008). ER heat shock protein (hsp) family members, BiP/GRP78 and GRP94, are not known to possess heat shock elements (HSE) in their promoters, however the yeast homolog of $\mathrm{BiP}(\mathrm{Kar} 2)$ was among the HSR target genes in the secretory pathway in UPR-deficient cells. Forty seven of 165 HSR target genes have functions in the secretory pathway in yeast. TTC17 is not conserved in yeast but TTC17 could be regulated under 
similar conditions in mammalian cells. TTC17 was found to be elevated in a mutant cell line that undergoes adaptive ER stress and is further increased upon raising the growth temperature (Pearse et al., 2008). This suggests that in mammalian systems, TTC17 expression could be activated to alleviate increased ER stress.

Initially, it would be of interest to characterize classic chaperones of the ER in the mutant cell line, MI8-5, both transcriptionally and translationally. In order to determine whether TTC17 directly regulates ER chaperones under stress conditions, we can delete TTC17 and monitor changes in expression of these chaperones. If there is no change in expression, global RNA and proteome profiling would be particularly useful to determine why TTC17 is up-regulated in the mutant cells, particularly at elevated growth temperature, as compared to wild type (WT).

The overexpression system used to previously identify putative interacting partners of TTC17 could have resulted in false positives. Genetically manipulating both WT CHO and MI8-5 cells to both endogenously tag TTC17, as well as deleting it would be particularly beneficial to understand its function. First, we can repeat affinity purification and subsequent proteomic analysis on endogenously tagged TTC17 to maintain native complexes. Alternatively, we could express TTC17 full-length and truncated constructs in a TTC17 knockout cell line to identify natural binding partners and isolate interactors of the TPR clusters alone. Given that interactions with TPR domains are often transient, we can design a TurboID plasmid to target TTC17, fulllength and individual TPR clusters, to the ER and efficiently label interacting partners by proximity labelling (Branon et al., 2018). TTC17 resembles a TPR-containing protein scaffold adaptor as it does not appear to possess any domains other than the TPRs. The 
most N-terminal TPR motif is directly followed by a coiled-coil domain, another proteinprotein interaction motif (Truebestein and Leonard, 2016).

Interestingly, TTC13 came down in an affinity purified complex with TTC17. TTC13 was identified by bioinformatic analysis as another TPR-containing protein that is predicted to possess an ER targeting signal sequence (Appendix A1A) (Hessa et al., 2007; Letunic and Bork, 2018; Nielsen, 2017). TTC13 has one predicted N-linked glycosylation site that does not appear to be recognized by endoglycosidases PNGaseF or EndoH (Appendix A1B) (Gupta, 2002). To this end, we genetically engineered two additional N-linked glycosylation sites at the N-terminus of TTC13 and observed a gel shift upon both PNGaseF and EndoH treatment indicating that TTC13 was targeted to the ER and has high mannose glycans similar to those of ER resident proteins (Appendix A1B). Immunofluorescence confocal microscopy of C-terminally tagged TTC13 shows that it co-localizes with ER marker, KDEL, and not the Golgi marker, GM130 (Appendix A1C). This confirms that TTC13 is an ER resident protein. Affinity purification of Cterminally tagged TTC13 did not interact with TTC17 suggesting that the interaction isolated in the TTC17 affinity purification may involve the C-terminus of TTC13 (Appendix A4). Analysis of TTC13 transcript regulation under ER stress conditions shows that it is not significantly up-regulated by stress however, it shares several putative interacting partners with TTC17 (Appendix A2 and A4).

To examine the exact nature of the interaction between TTC13 and TTC17, the proximity labeling (TurboID) system could also be applied to TTC13. Alternatively, we can endogenously tag TTC13 using genetic manipulation, affinity purify it from cells, conjugate it to beads and using different detergent conditions, isolate interacting partners

\section{1}


from cell lysates. Given that TTC17 is also heavily glycosylated, it would be more accurate to purify both TTC13 and TTC17 from mammalian cells using endogenous tagging and perform structural analysis. This can also be applied to each of the isolated TPR clusters within these proteins to identify their specific ligands.

Preliminary phenotypic analysis has been performed in cells and in animals to better understand both TTC13 and TTC17 function. To this end, eight shRNA constructs were designed for each TTC13 and TTC17, transfected into cells, and the cells were subsequently monitored for survival and morphological defects. No survival or morphology differences were observed. Analysis of TTC13 and TTC17 protein sequence conservation among commonly studied species, like TMTC analysis, revealed strong similarity to the TTC paralogues in Xenopus laevis. Morpholino sequences (Genetools LLC) were designed to match the ATG region of Xenopus laevis Ttc13 and Ttc17 to block protein translation of both allo allele ( $\mathrm{L}$ and $\mathrm{S}$ ). Individual morpholinos were microinjected into embryos at the 1-cell stage to ensure that every cell contained morpholino upon subsequent divisions. The injected embryos were allowed to develop as normal and a number of parameters were measured; including gastrulation, development, swimming phenotypes and survival. No significant differences were observed between morpholino injected embryos and non-injected controls suggesting that TTC13 and TTC17 do not play a major role in Xenopus development. However, it is possible that they play a functional role in stages beyond tadpole development or under certain environmental conditions. This study identified two novel ER TPR putative cochaperones, TTC13 and TTC17, and showed that TTC17 is regulated by or responds to 
cellular stress. Further biochemical and organismal studies are necessary to understand the role they play in maintaining cellular homeostasis and ER function.

\subsection{Summary}

In this thesis, we have further characterized six novel ER proteins, with multiple TPR motif arrangements, that were identified from an in silico search for putative ER organization and functional adaptor proteins. Greater understanding of TPR-containing proteins has divided the six novel proteins into two distinct classes, proteins containing elongated clusters of TPR motifs (TMTCs) and co-chaperone-like proteins (TTCs) with three to four consecutive motifs. Previous studies implied that TMTC1 and TMTC2 are novel regulators of calcium homeostasis and TMTC4 was recently shown to also regulate cellular calcium. Current data, including this thesis, identifies TMTC1-4 as putative Omannosyltransferases of the cadherin family of proteins, proteins which greatly depend on calcium for function. It is possible that the TMTCs modify different substrates and therefore could regulate calcium homeostasis through modification of key calcium players, shedding light on a complicated and intricately regulated process. TTC17 possesses two clusters of three and four TPRs, respectively, is regulated by ER stress and interacts with numerous chaperones indicating a co-chaperone-like role under stress conditions. Overall, a bioinformatic strategy was successful in identifying novel ER proteins with TPR motifs and biochemical characterization methods were successful in elucidating topology and putative function. While further analysis is required to understand their respective roles in maintaining ER homeostasis, this method can be applied to detect other common adaptor motifs such as the coiled-coil or ARM repeats in the ER or other organelles. 
$\begin{array}{llllllllllllll}\text { Fraction number: } & 1 & 2 & 3 & 4 & 5 & 6 & 7 & 8 & 9 & 10 & 11 & 12 & \mathrm{P}\end{array}$
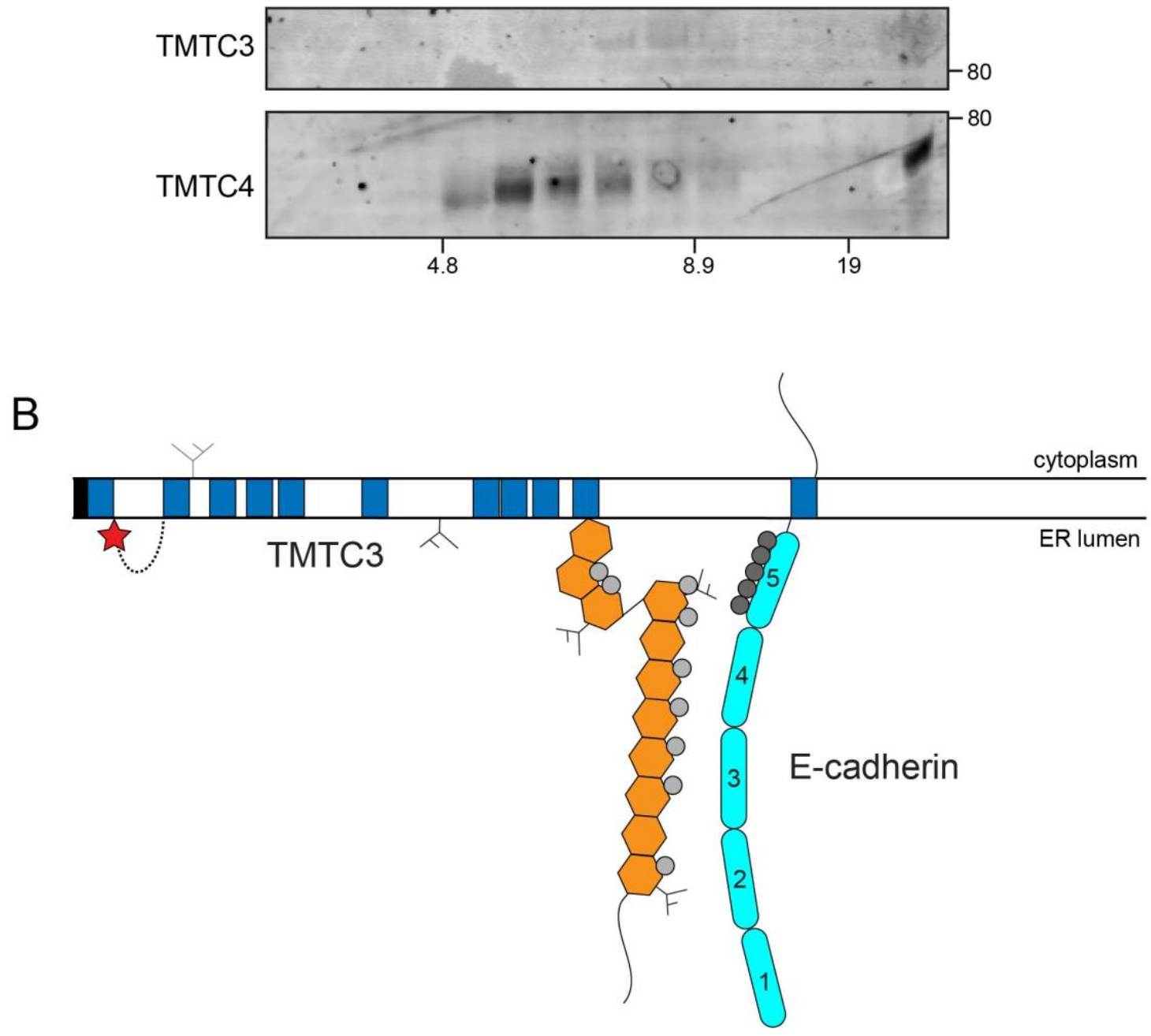

Figure 4.1. TMTC3 and TMTC4 reside in higher molecular weight complexes and may interact with substrate via conserved Asn. (A) HEK293T cells were transfected with Stagged TMTC3 or TMTC4 as indicated. Cells were lysed in MNT, and samples were layered on top of a continuous 10-40 \% sucrose gradient in MNT buffer prior to ultracentrifugation. Fractions were collected from the top of the gradient, and proteins were precipitated with trichloroacetic acid. Immunoblotting was then performed with appropriate antisera as described above. Sizing of detected complexes was estimated by comparison to the following standards designated at the bottom of the immunblots; bovine serum albumin $(4.6 \mathrm{~S}, 66 \mathrm{kDa})$; beta-amylase $(8.9 \mathrm{~S}, 200 \mathrm{kDa})$; and bovine thyroglobulin (19S, $669 \mathrm{kDa})(\mathrm{B})$ The ARM repeats of $\beta$-catenin interact with murine Ecadherin at the residues indicated by dark grey circles on extracellular cadherin (EC) domains (light blue). The ARM repeats interact via conserved Asn. The conserved Asn 
present on TMTC 3 TPR motifs at positions 6 and 9 of the TPR consensus are indicated in the light grey circles. These conserved Asn are hypothesized to help recruit substrate for O-mannosylation, which takes place adjacent to the membrane at the putative active site (red star) for TMTC3. 


\section{APPENDIX}

TTC13 IS A NOVEL ER RESIDENT TPR-CONTAINING PROTEIN 
A
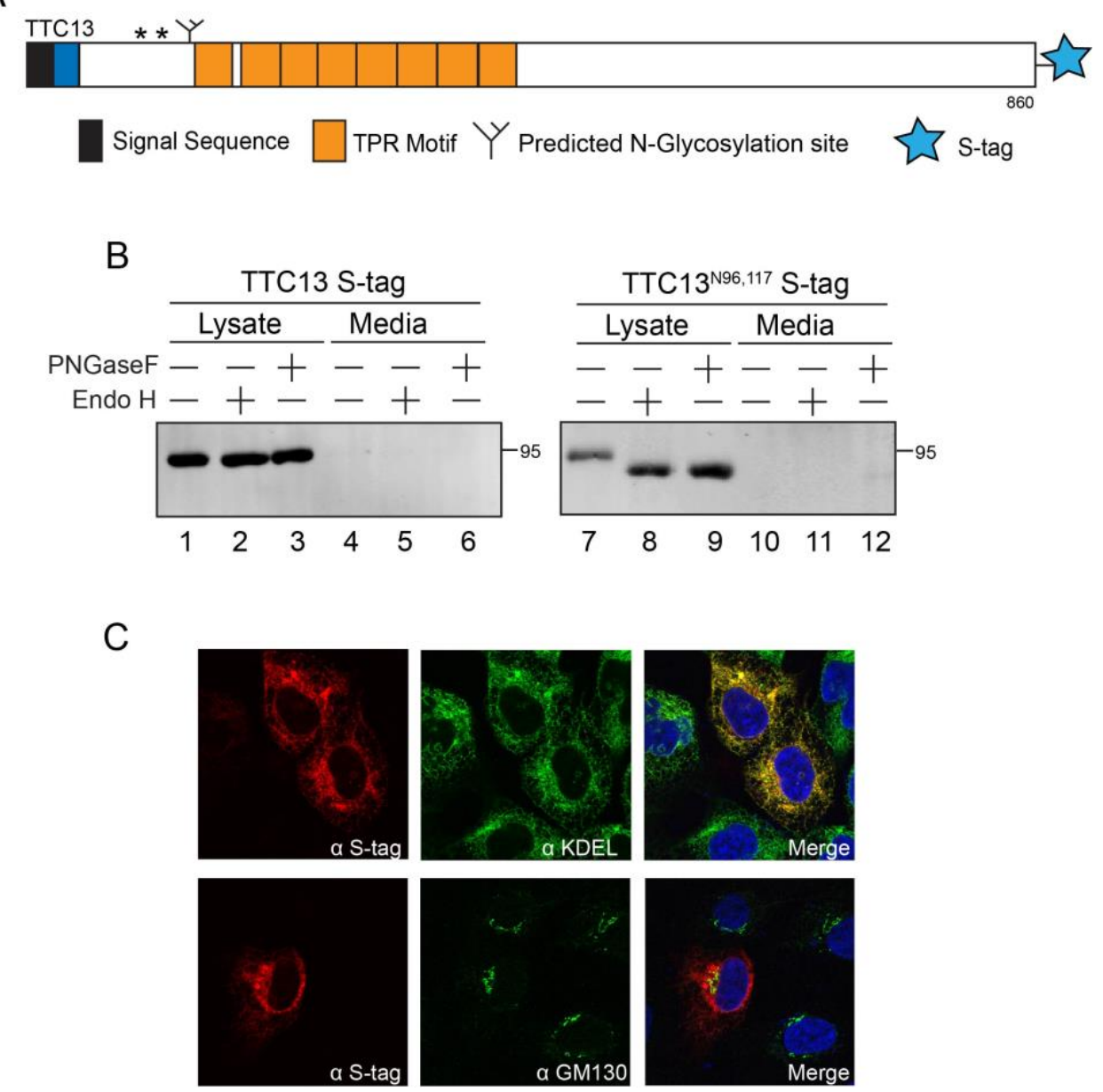

Figure A1. TTC13 is an ER resident TPR-containing protein. (A) Our screen for novel secretory adapter proteins identified TTC13 as a putative TPR-containing protein that was potentially targeted to the ER by an N-terminal signal sequence (black square). The position of tetratricopeptide repeats (TPRs) (orange squares) is designated and predicted $\mathrm{N}$-linked glycosylation sites are indicated by small, black, branched structures. Human TTC13 cDNA was C-terminally S-tagged (blue star) for biochemical studies. (B) HEK293T cells were transfected with S-tagged TTC13 WT or TTC13N96,117 and affinity purified from the cell lysate and media using S-protein agarose. Samples were then subjected to a glycosylation assay with either EndoH (lanes 2, 5, 8 and 11) or PNGaseF (lanes 3, 6, 9 and 12) digestion as indicated. Reducing sample buffer was added and the samples were analyzed by a 6\% SDS-PAGE and immunoblotted for the S-tag epitope. (C) Cellular localization of TTC13 was investigated by confocal microscopy. HEK293A cells were transfected with human TTC13 cDNA. Fixed cells were stained with S-tag, KDEL (ER) or GM130 (Golgi) antisera. Nuclei were visualized by DAPI staining (blue). 
A

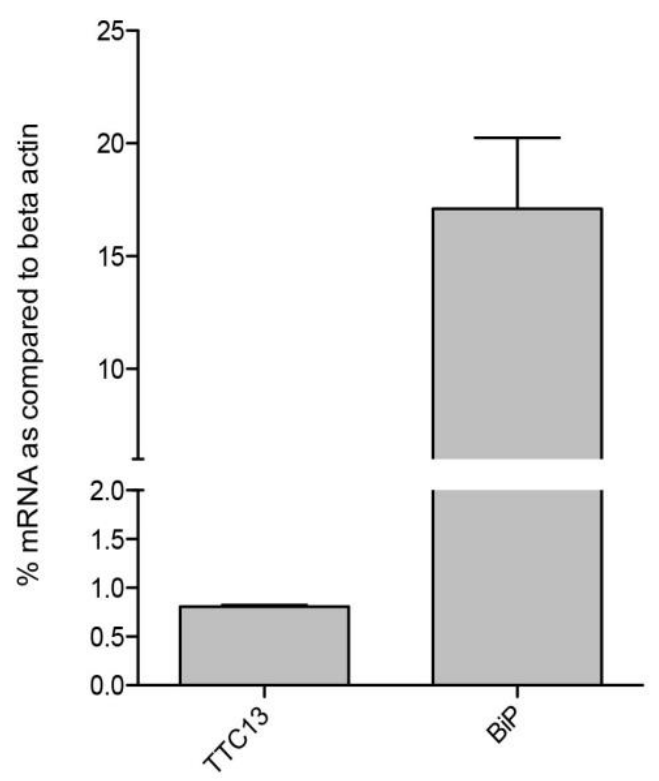

B

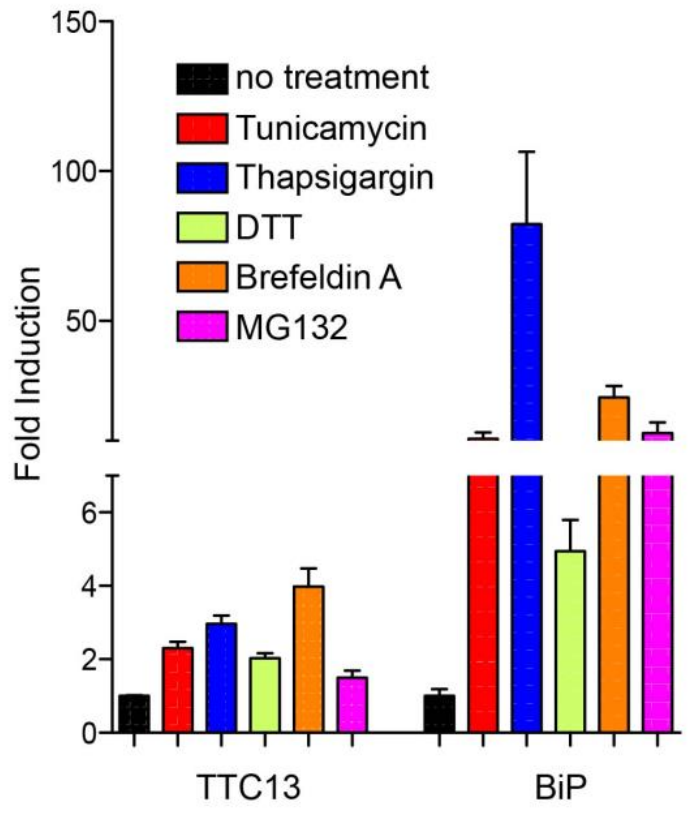

Figure A2. Basal transcript abundance and fold induction of TTC13 by ER stress. (A) RNA from HEK293A cells, grown under normal conditions, was harvested. RNA was reverse transcribed to cDNA followed by qRT-PCR with appropriate primers. Basal mRNA abundance was assessed using $\beta$-actin as a reference. Error bars represent standard deviation from at least three independent experiments. (B) HEK293A cells were treated with regular growth media or with $2 \mathrm{mM}$ DTT for $2 \mathrm{hr}, 1 \mu \mathrm{g} / \mathrm{mL}$ tunicamycin, 3 $\mu \mathrm{M}$ thapsigargin, $2.5 \mu \mathrm{g} / \mathrm{mL}$ brefeldin A or $2.5 \mu \mathrm{M}$ MG132 for $24 \mathrm{hr}$ prior to RNA purification. RNA was reverse transcribed to cDNA followed by qRT-PCR with appropriate primers, and changes in gene expression were calculated using $\beta$-actin as a reference. Error bars represent standard deviation from at least three independent experiments. 
A

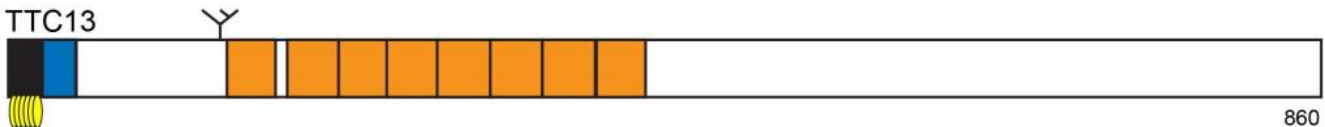

Signal Sequence $\square$ Hydrophobbic domain $Y$ Predicted N-Glycosylation site

$\bigcap$ Cysteine $\square$ TPR Motif

B

Sequence Conservation of TTC13

human TTC13MAPAGCCCCCCFWGGAVAAAGAARRVLLLLLLGVLSAGLRPGALATEHYSP

pan troglodytes MAPAG CCCCCCFWGGAVAAAGAARRVL LLLLGVLAGLRPGALATEHYSP

chlorocebus sabaeus MAPAGCCCCCCFWGGAVA|AAGAARRVLLLLLL AVLSAG RPGALATEHYSP

rhinopithecus roxellana MAPAGCCCCCCFWGGAVAAAGAARRVLLLLLLGVLSAG RPGALATEHYSP

macaca mulatta MAPAGCCCCCCFWGGAVAAAGAAR VLLLLLGVLSA RPGALATEHYSP macaca fascicularis MAPAGCCCCCCFWGGAVAAAGAAR VLLLLLLGVLSA RPGALATEHYSP condylura cristata MAPAGCCCCCC WGGAVAAAGAAR VLLLLLLGVLS G R GALATEHYSP oryctolagus cuniculus MAPAGCCCCCC CCCCCCWGGAVAAAAGAARRVL LLLLGVLSTA L PG LATEHYSP sus scrofa MAPAGCGCCGC_WGGAVAAAGAARRV LLLLLGVLSAG RPGALATEHYSP canis lupus familiaris MAPAGCCCC Y CCWGGAVAAA A RRVL LLLLGVLSAG RP ALATEHYSP

\begin{tabular}{|} 
SP \\
\begin{tabular}{|c|c|c|}
\multicolumn{1}{c}{ TP } & $\Delta \mathbf{G}$ \\
$45 \%$ & $94 \%$ & -1.303 \\
$49 \%$ & $93 \%$ & -1.027 \\
$81 \%$ & $94 \%$ & -0.598 \\
$82 \%$ & $93 \%$ & -0.173 \\
$49 \%$ & $97 \%$ & -0.691 \\
$49 \%$ & $97 \%$ & -0.691 \\
$44 \%$ & $93 \%$ & 0.315 \\
$65 \%$ & $97 \%$ & -1.394 \\
$62 \%$ & $48 \%$ & 1.170 \\
$75 \%$ & $87 \%$ & -0.095
\end{tabular}
\end{tabular}

$\mid=$ SignalP4.1
$\mid=$ TargetP1.1
= Both

C
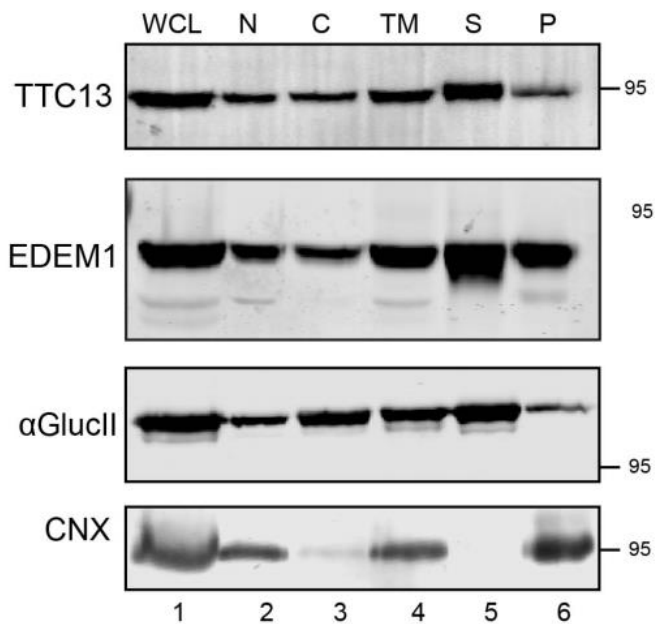

Figure A3. TTC13 is a soluble protein that resides within the ER lumen. (A) Our screen for novel secretory adapter proteins identified TTC13 as a putative TPR-containing protein that was potentially targeted to the ER by an N-terminal signal sequence (black 
square). TTC13 contains 6 consecutive Cys (yellow ovals) within its signal sequence. TTC13 is one of eight non-redundant proteins with this feature. (B) TTC13 putatively contains a hydrophobic domain (blue) that resides at the end of the predicted signal sequence. Analysis of the N-terminal TTC13 protein sequence among mammalian species revealed similar features. (C) HEK293T cells, transfected with TTC13 and EDEM1 S-tag, respectively, were homogenized and fractionated prior to alkaline extraction. The fractions collected were whole cell lysate (WCL), nucleus (N), cytosol (C), total membrane (TM), as well as supernatant (S) and pellet (P) fractions upon alkaline extraction of the TM. The WCL, C, and S fractions were then precipitated with trichloroacetic acid, and all samples were resuspended in sample buffer prior to the proteins being detected by immunoblotting with antisera directed against $\mathrm{S}$-tag, calnexin (CNX) and Glucosidase II $\alpha$ ( $\alpha$ GlucII). 


\begin{tabular}{|c|c|c|c|}
\hline Common Name & Mock & Unique Peptides & Corrected \\
\hline PDI & 0 & 14 & 14 \\
\hline $\mathrm{BiP}$ & 24 & 36 & 12 \\
\hline p180 & 4 & 14.5 & 11 \\
\hline Ribophorin I & 0 & 10 & 10 \\
\hline GRP94 & 11 & 21 & 10 \\
\hline Heterogeneous nuclear ribonucleoprotein $Q$ & 0 & 9 & 9 \\
\hline OST48 & 0 & 9 & 9 \\
\hline Cold shock domain containing E1 & 2 & 11 & 9 \\
\hline Acetyl-CoA acyltransferase, mitochondrial & 7 & 14 & 7 \\
\hline Peroxiredoxin-4 & 0 & 7 & 7 \\
\hline G protein-binding protein CRFG variant & 0 & 7 & 7 \\
\hline Drebrin-like protein & 0 & 7 & 7 \\
\hline $\begin{array}{l}\text { highly similar to Homo sapiens zinc finger } \\
\text { CCCH-type }\end{array}$ & 0 & 7 & 7 \\
\hline Importin-5 & 7 & 14 & 7 \\
\hline ERp57 & 8 & 15 & 7 \\
\hline SERCA2B & 10 & 16 & 6 \\
\hline$\alpha$ Glucosidase II & 0 & 6 & 6 \\
\hline Synaptotagmin binding & 0 & 6 & 6 \\
\hline highly similar to Dynamin-1-like protein & 0 & 5 & 5 \\
\hline Ribophorin II & 0 & 5 & 5 \\
\hline TCP-1- $\alpha$ & 14 & 19 & 5 \\
\hline Calreticulin & 0 & 5 & 5 \\
\hline $\operatorname{Sec} 61 \alpha$ & 1 & 6 & 5 \\
\hline $\begin{array}{l}\text { weakly similar to ATP-dependent RNA } \\
\text { helicase A }\end{array}$ & 0 & 5 & 5 \\
\hline GCP60 & 0 & 5 & 5 \\
\hline ERp72 & 1 & 6 & 5 \\
\hline Serine/arginine-rich protein-specific kinase 1 & 0 & 4 & 4 \\
\hline Sec31A & 0 & 4 & 4 \\
\hline
\end{tabular}

Figure A4. ER localized S-tagged TTC13 interacts with a number of chaperones. A shotgun liquid chromatography-mass spectrometry/mass spectrometry (LC-MS/MS) approach was used to identify potential binding partners of TTC17. HEK293T cells were transfected with TTC17-Stag plasmid followed by isotonic fractionation and ultracentrifugation to purify microsomes. The microsomes were resuspended in a buffer with detergent, and the tagged protein was affinity purified with S-protein agarose beads. The affinity purified complex was run on a short SDS-PAGE prior to in-gel trypsin digestion and silver staining. The peptides generated by trypsinization were loaded onto a LC-MS/MS instrument followed by peptide identification. Proteins were scored based on the number of unique peptides that were identified. The unique number of peptides was determined by averaging three independent experiments and then subsequently compared 
to mock. The corrected column represents the difference and is displayed in ascending order of number of peptides. 


\section{BIBLIOGRAPHY}

Adomako, A., Calvo, V., Biran, N., Osman, K., Chari, A., Paton, J.C., Paton, A.W., Moore, K., Schewe, D.M., and Aguirre-Ghiso, J.A. (2015). Identification of markers that functionally define a quiescent multiple myeloma cell sub-population surviving bortezomib treatment. BMC Cancer 15, 444.

Aebi, M., Bernasconi, R., Clerc, S., and Molinari, M. (2010). N-glycan structures: recognition and processing in the ER. Trends in Biochemical Sciences 35, 74-82.

Alder, N.N., Shen, Y., Brodsky, J.L., Hendershot, L.M., and Johnson, A.E. (2005). The molecular mechanisms underlying BiP-mediated gating of the Sec61 translocon of the endoplasmic reticulum. The Journal of Cell Biology 168, 389-399.

Allan, R.K., and Ratajczak, T. (2011). Versatile TPR domains accommodate different modes of target protein recognition and function. Cell Stress and Chaperones 16, 353367.

Andresen, C.A., Smedegaard, S., Sylvestersen, K.B., Svensson, C., Iglesias-Gato, D., Cazzamali, G., Nielsen, T.K., Nielsen, M.L., and Flores-Morales, A. (2014). Protein Interaction Screening for the Ankyrin Repeats and Suppressor of Cytokine Signaling (SOCS) Box (ASB) Family Identify Asb11 as a Novel Endoplasmic Reticulum Resident Ubiquitin Ligase. J. Biol. Chem. 289, 2043-2054.

Apweiler, R., Hermjakob, H., and Sharon, N. (1999). On the frequency of protein glycosylation, as deduced from analysis of the SWISS-PROT database11 Dedicated to Prof. Akira Kobata and Prof. Harry Schachter on the occasion of their 65th birthdays. Biochimica et Biophysica Acta (BBA) - General Subjects 1473, 4-8.

Ashby, M.C., and Tepikin, A.V. (2001). ER calcium and the functions of intracellular organelles. Seminars in Cell \& Developmental Biology 12, 11-17.

Assimon, V.A., Southworth, D.R., and Gestwicki, J.E. (2015). Specific binding of tetratricopeptide repeat (TPR) proteins to heat shock protein 70 (Hsp70) and heat shock protein 90 (Hsp90) is regulated by affinity and phosphorylation. Biochemistry 54, 71207131.

Bai, L., Kovach, A., You, Q., Kenny, A., and Li, H. (2019). Structure of the eukaryotic protein $\mathrm{O}$-mannosyltransferase Pmt1-Pmt2 complex. Nature Structural \& Molecular Biology 1.

Barkovich, A.J., Guerrini, R., Kuzniecky, R.I., Jackson, G.D., and Dobyns, W.B. (2012). A developmental and genetic classification for malformations of cortical development: update 2012. Brain 135, 1348-1369. 
Barlowe, C. (1998). COPII and selective export from the endoplasmic reticulum. Biochimica et Biophysica Acta (BBA) - Molecular Cell Research 1404, 67-76.

Barresi, R., and Campbell, K.P. (2006). Dystroglycan: from biosynthesis to pathogenesis of human disease. Journal of Cell Science 119, 199-207.

Bause, E., and Lehle, L. (1979). Enzymatic N-Glycosylation and O-Glycosylation of Synthetic Peptide Acceptors by Dolichol-Linked Sugar Derivatives in Yeast. European Journal of Biochemistry 101, 531-540.

Beltrán-Valero de Bernabé, D., Currier, S., Steinbrecher, A., Celli, J., van Beusekom, E., van der Zwaag, B., Kayserili, H., Merlini, L., Chitayat, D., Dobyns, W.B., et al. (2002). Mutations in the O-mannosyltransferase gene POMT1 give rise to the severe neuronal migration disorder Walker-Warburg syndrome. Am. J. Hum. Genet. 71, 1033-1043.

Benyair, R., Ogen-Shtern, N., and Lederkremer, G.Z. (2015a). Glycan regulation of ERassociated degradation through compartmentalization. Seminars in Cell \& Developmental Biology 41, 99-109.

Benyair, R., Ogen-Shtern, N., Mazkereth, N., Shai, B., Ehrlich, M., and Lederkremer, G.Z. (2015b). Mammalian ER mannosidase I resides in quality control vesicles, where it encounters its glycoprotein substrates. Mol Biol Cell 26, 172-184.

Blobel, G. (1980). Intracellular protein topogenesis. PNAS 77, 1496-1500.

Boehning, D., Patterson, R.L., Sedaghat, L., Glebova, N.O., Kurosaki, T., and Snyder, S.H. (2003). Cytochrome c binds to inositol $(1,4,5)$ trisphosphate receptors, amplifying calcium-dependent apoptosis. Nat. Cell Biol. 5, 1051-1061.

Bontems, F., Fish, R.J., Borlat, I., Lembo, F., Chocu, S., Chalmel, F., Borg, J.-P., Pineau, C., Neerman-Arbez, M., Bairoch, A., et al. (2014). C2orf62 and TTC17 Are Involved in Actin Organization and Ciliogenesis in Zebrafish and Human. PLOS ONE 9, e86476.

Branon, T.C., Bosch, J.A., Sanchez, A.D., Udeshi, N.D., Svinkina, T., Carr, S.A., Feldman, J.L., Perrimon, N., and Ting, A.Y. (2018). Efficient proximity labeling in living cells and organisms with TurboID. Nature Biotechnology 36, 880-887.

Brasch, J., Harrison, O.J., Honig, B., and Shapiro, L. (2012). Thinking outside the cell: how cadherins drive adhesion. Trends in Cell Biology 22, 299-310.

Bräuer, P., Parker, J.L., Gerondopoulos, A., Zimmermann, I., Seeger, M.A., Barr, F.A., and Newstead, S. (2019). Structural basis for $\mathrm{pH}$-dependent retrieval of ER proteins from the Golgi by the KDEL receptor. Science 363, 1103-1107.

Brehme, M., Voisine, C., Rolland, T., Wachi, S., Soper, J.H., Zhu, Y., Orton, K., Villella, A., Garza, D., Vidal, M., et al. (2014). A Chaperome Subnetwork Safeguards Proteostasis in Aging and Neurodegenerative Disease. Cell Reports 9, 1135-1150.

\section{4}


Brini, M., and Carafoli, E. (2009). Calcium Pumps in Health and Disease. Physiological Reviews 89, 1341-1378.

Budnik, A., and Stephens, D.J. (2009). ER exit sites - Localization and control of COPII vesicle formation. FEBS Letters 583, 3796-3803.

Bunney, T.D., Cole, A.R., Broncel, M., Esposito, D., Tate, E.W., and Katan, M. (2014). Crystal Structure of the Human, FIC-Domain Containing Protein HYPE and Implications for Its Functions. Structure 22, 1831-1843.

Bürkle, A. (2001). Posttranslational Modification. In Encyclopedia of Genetics, S. Brenner, and J.H. Miller, eds. (New York: Academic Press), p. 1533.

Cao, D., Jiao, X., Liu, X., Hennis, A., Leske, M.C., Nemesure, B., and Hejtmancik, J.F. (2012). CDKN2B Polymorphism Is Associated with Primary Open-Angle Glaucoma (POAG) in the Afro-Caribbean Population of Barbados, West Indies. PLOS ONE 7, e39278.

Caplan, A.J. (2003). What is a co-chaperone? Cell Stress Chaperones 8, 105-107.

Caro, L.G. (1964). Protein Synthesis, Storage, and Discharge in the Pancreatic Exocrine Cell: An Autoradiographic Study. The Journal of Cell Biology 20, 473-495.

Carvalho, P., Goder, V., and Rapoport, T.A. (2006). Distinct Ubiquitin-Ligase Complexes Define Convergent Pathways for the Degradation of ER Proteins. Cell 126, 361-373.

Casas, C. (2017). GRP78 at the Centre of the Stage in Cancer and Neuroprotection. Front. Neurosci. 11.

Cavallaro, U., and Christofori, G. (2004). Cell adhesion and signalling by cadherins and Ig-CAMs in cancer. Nature Reviews Cancer 4, 118.

Cerveny, L., Straskova, A., Dankova, V., Hartlova, A., Ceckova, M., Staud, F., and Stulik, J. (2013). Tetratricopeptide Repeat Motifs in the World of Bacterial Pathogens: Role in Virulence Mechanisms. Infection and Immunity 81, 629-635.

Chartron, J.W., Gonzalez, G.M., and Clemons, W.M. (2011). A Structural Model of the Sgt 2 Protein and Its Interactions with Chaperones and the Get4/Get5 Complex. J. Biol. Chem. 286, 34325-34334.

Chen, W., Helenius, J., Braakman, I., and Helenius, A. (1995). Cotranslational folding and calnexin binding during glycoprotein synthesis. PNAS 92, 6229-6233.

Christianson, J.C., Shaler, T.A., Tyler, R.E., and Kopito, R.R. (2008). OS-9 and GRP94 deliver mutant alpha1-antitrypsin to the Hrd1-SEL1L ubiquitin ligase complex for ERAD. Nat. Cell Biol. 10, 272-282. 
Conti, E., Uy, M., Leighton, L., Blobel, G., and Kuriyan, J. (1998). Crystallographic Analysis of the Recognition of a Nuclear Localization Signal by the Nuclear Import Factor Karyopherin $\alpha$. Cell 94, 193-204.

Cousin, H., DeSimone, D.W., and Alfandari, D. (2008). PACSIN2 regulates cell adhesion during gastrulation in Xenopus laevis. Developmental Biology 319, 86-99.

D'Alessio, C., Caramelo, J.J., and Parodi, A.J. (2010). UDP-GlC:glycoprotein glucosyltransferase-glucosidase II, the ying-yang of the ER quality control. Seminars in Cell \& Developmental Biology 21, 491-499.

D'Andrea, L.D., and Regan, L. (2003). TPR proteins: the versatile helix. Trends in Biochemical Sciences 28, 655-662.

Daniels, R., Kurowski, B., Johnson, A.E., and Hebert, D.N. (2003). N-Linked Glycans Direct the Cotranslational Folding Pathway of Influenza Hemagglutinin. Molecular Cell 11, 79-90.

Darby, J.F., Krysztofinska, E.M., Simpson, P.J., Simon, A.C., Leznicki, P., Sriskandarajah, N., Bishop, D.S., Hale, L.R., Alfano, C., Conte, M.R., et al. (2014). Solution Structure of the SGTA Dimerisation Domain and Investigation of Its Interactions with the Ubiquitin-Like Domains of BAG6 and UBL4A. PLOS ONE 9, e113281.

Della-Morte, D., Beecham, A., Rundek, T., Wang, L., McClendon, M.S., Slifer, S., Blanton, S.H., Di Tullio, M.R., and Sacco, R.L. (2011). A follow-up study for left ventricular mass on chromosome 12p11 identifies potential candidate genes. BMC Med Genet 12, 100.

DeSimone, D.W., Dzamba, B., and Davidson, L.A. (2007). Using Xenopus Embryos to Investigate Integrin Function. In Methods in Enzymology, (Academic Press), pp. 403414.

Dudek, J., Benedix, J., Cappel, S., Greiner, M., Jalal, C., Müller, L., and Zimmermann, R. (2009). Functions and pathologies of BiP and its interaction partners. Cell. Mol. Life Sci. $66,1556-1569$.

Dutta, S., and Tan, Y.-J. (2008). Structural and Functional Characterization of Human SGT and Its Interaction with Vpu of the Human Immunodeficiency Virus Type 1,. Biochemistry 47, 10123-10131.

Ecker, M., Mrsa, V., Hagen, I., Deutzmann, R., Strahl, S., and Tanner, W. (2003). OMannosylation precedes and potentially controls the $\mathrm{N}$-glycosylation of a yeast cell wall glycoprotein. EMBO Reports 4, 628-632. 
Ellgaard, L., and Helenius, A. (2003). Quality control in the endoplasmic reticulum. Nature Reviews Molecular Cell Biology 4, 181-191.

Emanuelsson, O., Nielsen, H., Brunak, S., and von Heijne, G. (2000). Predicting Subcellular Localization of Proteins Based on their N-terminal Amino Acid Sequence. Journal of Molecular Biology 300, 1005-1016.

English, A.R., Zurek, N., and Voeltz, G.K. (2009). Peripheral ER Structure to Function. Curr Opin Cell Biol 21, 596-602.

Faber, P.W., Barnes, G.T., Srinidhi, J., Chen, J., Gusella, J.F., and MacDonald, M.E. (1998). Huntingtin interacts with a family of WW domain proteins. Human Molecular Genetics 7, 1463-1474.

Fan, A.C.Y., Bhangoo, M.K., and Young, J.C. (2006). Hsp90 Functions in the Targeting and Outer Membrane Translocation Steps of Tom70-mediated Mitochondrial Import. J. Biol. Chem. 281, 33313-33324.

Farhan, S.M.K., Nixon, K.C.J., Everest, M., Edwards, T.N., Long, S., Segal, D., Knip, M.J., Arts, H.H., Chakrabarti, R., Wang, J., et al. (2017). Identification of a novel synaptic protein, TMTC3, involved in periventricular nodular heterotopia with intellectual disability and epilepsy. Hum Mol Genet 26, 4278-4289.

Freeze, H.H., and Elbein, A.D. (2009). Glycosylation Precursors. In Essentials of Glycobiology, A. Varki, R.D. Cummings, J.D. Esko, H.H. Freeze, P. Stanley, C.R. Bertozzi, G.W. Hart, and M.E. Etzler, eds. (Cold Spring Harbor (NY): Cold Spring Harbor Laboratory Press), p.

Fujimori, T., Suno, R., Iemura, S.-I., Natsume, T., Wada, I., and Hosokawa, N. (2017). Endoplasmic reticulum proteins SDF2 and SDF2L1 act as components of the BiP chaperone cycle to prevent protein aggregation. Genes Cells 22, 684-698.

Garcia-Pino, A., Zenkin, N., and Loris, R. (2014). The many faces of Fic: structural and functional aspects of Fic enzymes. Trends in Biochemical Sciences 39, 121-129.

Gardner, R.G., Swarbrick, G.M., Bays, N.W., Cronin, S.R., Wilhovsky, S., Seelig, L., Kim, C., and Hampton, R.Y. (2000). Endoplasmic Reticulum Degradation Requires Lumen to Cytosol Signaling: Transmembrane Control of Hrd1p by Hrd3p. J Cell Biol $151,69-82$.

Gauss, R., Jarosch, E., Sommer, T., and Hirsch, C. (2006). A complex of Yos9p and the HRD ligase integrates endoplasmic reticulum quality control into the degradation machinery. Nat. Cell Biol. 8, 849-854.

Gentzsch, M., and Tanner, W. (1996). The PMT gene family: protein O-glycosylation in Saccharomyces cerevisiae is vital. The EMBO Journal 15, 5752-5759. 
Gentzsch, M., and Tanner, W. (1997). Protein-O-glycosylation in yeast: protein-specific mannosyltransferases. Glycobiology 7, 481-486.

Gentzsch, M., Immervoll, T., and Tanner, W. (1995). Protein O-glycosylation in Saccharomyces cerevisiae: the protein O-mannosyltransferases Pmt1p and Pmt2p function as heterodimer. FEBS Letters 377, 128-130.

Gidalevitz, T., Stevens, F., and Argon, Y. (2013). Orchestration of secretory protein folding by ER chaperones. Biochimica et Biophysica Acta (BBA) - Molecular Cell Research 1833, 2410-2424.

Gilchrist, A., Au, C.E., Hiding, J., Bell, A.W., Fernandez-Rodriguez, J., Lesimple, S., Nagaya, H., Roy, L., Gosline, S.J.C., Hallett, M., et al. (2006). Quantitative Proteomics Analysis of the Secretory Pathway. Cell 127, 1265-1281.

Giorgi, C., De Stefani, D., Bononi, A., Rizzuto, R., and Pinton, P. (2009). Structural and functional link between the mitochondrial network and the endoplasmic reticulum. The International Journal of Biochemistry \& Cell Biology 41, 1817-1827.

Girrbach, V., Zeller, T., Priesmeier, M., and Strahl-Bolsinger, S. (2000). StructureFunction Analysis of the Dolichyl Phosphate-Mannose: Protein O-Mannosyltransferase ScPmt1p. J. Biol. Chem. 275, 19288-19296.

Glaser, T., Arnaud Sampaio, V.F., Lameu, C., and Ulrich, H. (2018). Calcium signalling: A common target in neurological disorders and neurogenesis. Seminars in Cell \& Developmental Biology.

Good, M.C., Zalatan, J.G., and Lim, W.A. (2011). Scaffold proteins: hubs for controlling the flow of cellular information. Science 332, 680-686.

Graham, J.B., Canniff, N.P., and Hebert, D.N. (2019). TPR-containing proteins control protein organization and homeostasis for the endoplasmic reticulum. Critical Reviews in Biochemistry and Molecular Biology 54, 103-118.

Grove, T.Z., Cortajarena, A.L., and Regan, L. (2008). Ligand binding by repeat proteins: natural and designed. Current Opinion in Structural Biology 18, 507-515.

Guerreiro, P., Barreiros, T., Soares, H., Cyrne, L., Silva, A.M.E., and Rodrigues-Pousada, C. (1996). Sequencing of a $17.6 \mathrm{~kb}$ segment on the right arm of yeast chromosome VII reveals 12 ORFs, including CCT, ADE3 and TR-I genes, homologues of the yeast PMT and EF1G genes, of the human and bacterial electron-transferring flavoproteins ( $\beta$-chain) and of the Escherichia coli phosphoserine phosphohydrolase, and five new ORFs. Yeast $12,273-280$. 
Guillen-Ahlers, H., Erbe, C.B., Chevalier, F.D., Montoya, M.J., Zimmerman, K.D., Langefeld, C.D., Olivier, M., and Runge, C.L. (2018). TMTC2 variant associated with sensorineural hearing loss and auditory neuropathy spectrum disorder in a family dyad. Mol Genet Genomic Med 6, 653-659.

Gupta, R. (2002). Prediction of Glycosylation Across the Human Proteome and the Correlation to Protein Function. Pacific Symposium on Biocomputing 310-322.

Haines, N., and Irvine, K.D. (2003). Glycosylation regulates Notch signalling. Nat Rev Mol Cell Biol 4, 786-797.

Halbleib, J.M., and Nelson, W.J. (2006). Cadherins in development: cell adhesion, sorting, and tissue morphogenesis. Genes Dev. 20, 3199-3214.

Hales, C.N., Luzio, J.P., Chandler, J.A., and Herman, L. (1974). Localization of calcium in the smooth endoplasmic reticulum of rat isolated fat cells. J. Cell. Sci. 15, 1-15.

Harada, Y., Li, H., Li, H., and Lennarz, W.J. (2009). Oligosaccharyltransferase directly binds to ribosome at a location near the translocon-binding site. PNAS 106, 6945-6949.

Harkness, T.A., Nargang, F.E., Klei, I. van der, Neupert, W., and Lill, R. (1994). A crucial role of the mitochondrial protein import receptor MOM19 for the biogenesis of mitochondria. The Journal of Cell Biology 124, 637-648.

Hart, G.W., Housley, M.P., and Slawson, C. (2007). Cycling of O-linked $\beta-N$ acetylglucosamine on nucleocytoplasmic proteins. Nature 446, 1017-1022.

Hartl, F.U., Bracher, A., and Hayer-Hartl, M. (2011). Molecular chaperones in protein folding and proteostasis. Nature 475, 324-332.

Hayashi, T., Rizzuto, R., Hajnoczky, G., and Su, T.-P. (2009). MAM: more than just a housekeeper. Trends in Cell Biology 19, 81-88.

Hebert, D.N., and Molinari, M. (2007). In and Out of the ER: Protein Folding, Quality Control, Degradation, and Related Human Diseases. Physiological Reviews 87, 13771408 .

Hebert, D.N., Lamriben, L., Powers, E.T., and Kelly, J.W. (2014). The intrinsic and extrinsic effects of $\mathrm{N}$-linked glycans on glycoproteostasis. Nature Chemical Biology 10, 902-910.

Helenius, A., and Aebi, M. (2004). Roles of N-Linked Glycans in the Endoplasmic Reticulum. Annual Review of Biochemistry 73, 1019-1049.

Hessa, T., Meindl-Beinker, N.M., Bernsel, A., Kim, H., Sato, Y., Lerch-Bader, M., Nilsson, I., White, S.H., and von Heijne, G. (2007). Molecular code for transmembranehelix recognition by the Sec61 translocon. Nature 450, 1026-1030. 
Hideaki, I., and Yohtalou, T. (1991). The stress (heat shock) proteins. International Journal of Biochemistry 23, 1185-1191.

Huber, A.H., and Weis, W.I. (2001). The Structure of the $\beta$-Catenin/E-Cadherin Complex and the Molecular Basis of Diverse Ligand Recognition by $\beta$-Catenin. Cell 105, 391-402.

Immervoll, T., Gentzsch, M., and Tanner, W. (1995). PMT3 and PMT4, two new members of the protein-O-mannosyltransferase gene family of Saccharomyces cerevisiae. Yeast $11,1345-1351$.

Imperiali, B., and Hendrickson, T.L. (1995). Asparagine-linked glycosylation: Specificity and function of oligosaccharyl transferase. Bioorganic \& Medicinal Chemistry 3, 15651578 .

Itskanov, S., and Park, E. (2019). Structure of the posttranslational Sec proteintranslocation channel complex from yeast. Science 363, 84-87.

Iyer, S.P.N., and Hart, G.W. (2003). Roles of the Tetratricopeptide Repeat Domain in OGlcNAc Transferase Targeting and Protein Substrate Specificity. J. Biol. Chem. 278, 24608-24616.

Janik, M.E., Lityńska, A., and Vereecken, P. (2010). Cell migration-The role of integrin glycosylation. Biochimica et Biophysica Acta (BBA) - General Subjects 1800, 545-555.

Javadi, Y., and Main, E.R.G. (2009). Exploring the folding energy landscape of a series of designed consensus tetratricopeptide repeat proteins. PNAS 106, 17383-17388.

Jayaprakash, N.G., and Surolia, A. (2017). Role of glycosylation in nucleating protein folding and stability. Biochemical Journal 474, 2333-2347.

Jensen, D., and Schekman, R. (2011). COPII-mediated vesicle formation at a glance. Journal of Cell Science 124, 1-4.

Jeong, H., Sim, H.J., Song, E.K., Lee, H., Ha, S.C., Jun, Y., Park, T.J., and Lee, C. (2016). Crystal structure of SEL1L: Insight into the roles of SLR motifs in ERAD pathway. Scientific Reports 6, 20261.

Jerber, J., Zaki, M.S., Al-Aama, J.Y., Rosti, R.O., Ben-Omran, T., Dikoglu, E., Silhavy, J.L., Caglar, C., Musaev, D., Albrecht, B., et al. (2016). Biallelic Mutations in TMTC3, Encoding a Transmembrane and TPR-Containing Protein, Lead to Cobblestone Lissencephaly. Am J Hum Genet 99, 1181-1189.

Jiménez, B., Ugwu, F., Zhao, R., Ortí, L., Makhnevych, T., Pineda-Lucena, A., and Houry, W.A. (2012). Structure of Minimal Tetratricopeptide Repeat Domain Protein Tah1 Reveals Mechanism of Its Interaction with Pih1 and Hsp90. J. Biol. Chem. 287, 5698-5709. 
Jínek, M., Rehwinkel, J., Lazarus, B.D., Izaurralde, E., Hanover, J.A., and Conti, E. (2004). The superhelical TPR-repeat domain of O-linked GlcNAc transferase exhibits structural similarities to importin alpha. Nat. Struct. Mol. Biol. 11, 1001-1007.

Johnson, A.E., and van Waes, M.A. (1999). The Translocon: A Dynamic Gateway at the ER Membrane. Annual Review of Cell and Developmental Biology 15, 799-842.

Jonikas, M.C., Collins, S.R., Denic, V., Oh, E., Quan, E.M., Schmid, V., Weibezahn, J., Schwappach, B., Walter, P., Weissman, J.S., et al. (2009). Comprehensive Characterization of Genes Required for Protein Folding in the Endoplasmic Reticulum. Science 323, 1693-1697.

Joshi, H.J., Narimatsu, Y., Schjoldager, K.T., Tytgat, H.L.P., Aebi, M., Clausen, H., and Halim, A. (2018). SnapShot: O-Glycosylation Pathways across Kingdoms. Cell 172, 632632.e2.

Kajander, T., Cortajarena, A.L., Mochrie, S., and Regan, L. (2007). Structure and stability of designed TPR protein superhelices: unusual crystal packing and implications for natural TPR proteins. Acta Crystallographica Section D Biological Crystallography $63,800-811$.

Kalantari, F., Bergeron, J.J.M., and Nilsson, T. (2010). Biogenesis of lipid droplets - how cells get fatter. Molecular Membrane Biology 27, 462-468.

Kaneko, M., and Nomura, Y. (2003). ER signaling in unfolded protein response. Life Sciences 74, 199-205.

Karpenahalli, M.R., Lupas, A.N., and Söding, J. (2007). TPRpred: a tool for prediction of TPR-, PPR- and SEL1-like repeats from protein sequences. BMC Bioinformatics $8,2$.

Klopfenstein, D.R., Klumperman, J., Lustig, A., Kammerer, R.A., Oorschot, V., and Hauri, H.-P. (2001). Subdomain-Specific Localization of Climp-63 (P63) in the Endoplasmic Reticulum Is Mediated by Its Luminal $\alpha$-Helical Segment. The Journal of Cell Biology 153, 1287-1300.

Kopito, R.R., and Sitia, R. (2000). Aggresomes and Russell bodies: Symptoms of cellular indigestion? EMBO Rep 1, 225-231.

Lackner, D.H., Carré, A., Guzzardo, P.M., Banning, C., Mangena, R., Henley, T., Oberndorfer, S., Gapp, B.V., Nijman, S.M.B., Brummelkamp, T.R., et al. (2015). A generic strategy for CRISPR-Cas9-mediated gene tagging. Nature Communications 6.

Lairson, L.L., Henrissat, B., Davies, G.J., and Withers, S.G. (2008).

Glycosyltransferases: Structures, Functions, and Mechanisms. Annual Review of

Biochemistry 77, 521-555. 
Lamriben, L., Graham, J.B., Adams, B.M., and Hebert, D.N. (2016). $N$-Glycan-based ER Molecular Chaperone and Protein Quality Control System: The Calnexin Binding Cycle: The Calnexin Binding Cycle. Traffic 17, 308-326.

Larsen, I.S.B., Narimatsu, Y., Joshi, H.J., Siukstaite, L., Harrison, O.J., Brasch, J., Goodman, K.M., Hansen, L., Shapiro, L., Honig, B., et al. (2017a). Discovery of an Omannosylation pathway selectively serving cadherins and protocadherins. PNAS 114, 11163-11168.

Larsen, I.S.B., Narimatsu, Y., Joshi, H.J., Yang, Z., Harrison, O.J., Brasch, J., Shapiro, L., Honig, B., Vakhrushev, S.Y., Clausen, H., et al. (2017b). Mammalian $O$ mannosylation of cadherins and plexins is independent of protein $O$ mannosyltransferases 1 and 2. Journal of Biological Chemistry 292, 11586-11598.

Larsen, I.S.B., Narimatsu, Y., Clausen, H., Joshi, H.J., and Halim, A. (2019). Multiple distinct O-Mannosylation pathways in eukaryotes. Current Opinion in Structural Biology $56,171-178$.

Laskey, R.A., Mills, A.D., Gurdon, J.B., and Partington, G.A. (1977). Protein synthesis in oocytes of xenopus laevis is not regulated by the supply of messenger RNA. Cell 11 , $345-351$.

Lazarus, M.B., Nam, Y., Jiang, J., Sliz, P., and Walker, S. (2011). Structure of human $O$ GlcNAc transferase and its complex with a peptide substrate. Nature 469, 564-567.

Letunic, I., and Bork, P. (2018). 20 years of the SMART protein domain annotation resource. Nucleic Acids Res 46, D493-D496.

Levine, T., and Loewen, C. (2006). Inter-organelle membrane contact sites: through a glass, darkly. Curr. Opin. Cell Biol. 18, 371-378.

Leznicki, P., Korac-Prlic, J., Kliza, K., Husnjak, K., Nyathi, Y., Dikic, I., and High, S. (2015). Binding of SGTA to Rpn13 selectively modulates protein quality control. J Cell Sci $128,3187-3196$.

Li, Y., and Camacho, P. (2004). Ca2+-dependent redox modulation of SERCA $2 b$ by ERp57. The Journal of Cell Biology 164, 35-46.

Li, J., Akil, O., Rouse, S.L., McLaughlin, C.W., Matthews, I.R., Lustig, L.R., Chan, D.K., and Sherr, E.H. (2018). Deletion of Tmtc4 activates the unfolded protein response and causes postnatal hearing loss. J Clin Invest 128, 5150-5162.

Li, S., Iakoucheva, L.M., Mooney, S.D., and Radivojac, P. (2010). Loss of Posttranslatinal Modification sites in Disease. Pac Symp Biocomput 337-347. 
Liberek, K., Marszalek, J., Ang, D., Georgopoulos, C., and Zylicz, M. (1991).

Escherichia coli DnaJ and GrpE heat shock proteins jointly stimulate ATPase activity of DnaK. PNAS 88, 2874-2878.

Liu, J., and Mushegian, A. (2003). Three monophyletic superfamilies account for the majority of the known glycosyltransferases. Protein Sci 12, 1418-1431.

Liu, Y., and Chang, A. (2008). Heat shock response relieves ER stress. The EMBO Journal 27, 1049-1059.

Lodish, H., Berk, A., Zipursky, S.L., Matsudaira, P., Baltimore, D., and Darnell, J. (2000). Cell-Cell Adhesion and Communication. Molecular Cell Biology. 4th Edition.

Loibl, M., and Strahl, S. (2013). Protein O-mannosylation: What we have learned from baker's yeast. Biochimica et Biophysica Acta (BBA) - Molecular Cell Research 1833, 2438-2446.

Lommel, M., and Strahl, S. (2009). Protein O-mannosylation: Conserved from bacteria to humans. Glycobiology 19, 816-828.

Lommel, M., Schott, A., Jank, T., Hofmann, V., and Strahl, S. (2011). A Conserved Acidic Motif Is Crucial for Enzymatic Activity of Protein O-Mannosyltransferases. J Biol Chem 286, 39768-39775.

Lommel, M., Winterhalter, P.R., Willer, T., Dahlhoff, M., Schneider, M.R., Bartels, M.F., Renner-Müller, I., Ruppert, T., Wolf, E., and Strahl, S. (2013). Protein Omannosylation is crucial for E-cadherin-mediated cell adhesion. PNAS 110, 21024 21029.

Lubas, W.A., Frank, D.W., Krause, M., and Hanover, J.A. (1997). O-Linked GlcNAc Transferase Is a Conserved Nucleocytoplasmic Protein Containing Tetratricopeptide Repeats. J. Biol. Chem. 272, 9316-9324.

Lunz, V., Romanin, C., and Frischauf, I. (2019). STIM1 activation of Orai1. Cell Calcium 77, 29-38.

Lussier, M., Gentzsch, M., Sdicu, A.-M., Bussey, H., and Tanner, W. (1995). Protein OGlycosylation in Yeast The Pmt2 Gene Specifies a Second Protein O-

mannosyltransferase that Functions in addition to the Pmt1-encoded Activity. J. Biol. Chem. 270, 2770-2775.

Lynes, E.M., and Simmen, T. (2011). Urban planning of the endoplasmic reticulum (ER): How diverse mechanisms segregate the many functions of the ER. Biochimica et Biophysica Acta (BBA) - Molecular Cell Research 1813, 1893-1905. 
Lynes, E.M., Raturi, A., Shenkman, M., Sandoval, C.O., Yap, M.C., Wu, J., Janowicz, A., Myhill, N., Benson, M.D., Campbell, R.E., et al. (2013). Palmitoylation is the switch that assigns calnexin to quality control or ER Ca2+ signaling. J Cell Sci 126, 3893-3903.

Madsen, M.B., Kogelman, L.J.A., Kadarmideen, H.N., and Rasmussen, H.B. (2018). Systems genetics analysis of pharmacogenomics variation during antidepressant treatment. The Pharmacogenomics Journal 18, 144-152.

Magliery, T.J., and Regan, L. (2005). Sequence variation in ligand binding sites in proteins. BMC Bioinformatics 6, 240.

Mahat, D.B., Salamanca, H.H., Duarte, F.M., Danko, C.G., and Lis, J.T. (2016). Mammalian Heat Shock Response and Mechanisms Underlying Its Genome-wide Transcriptional Regulation. Molecular Cell 62, 63-78.

Maitre, J.-L., and Heisenberg, C.-P. (2013). Three Functions of Cadherins in Cell Adhesion. Current Biology 23, R626-R633.

Martínez-Lumbreras, S., Krysztofinska, E.M., Thapaliya, A., Spilotros, A., MatakVinkovic, D., Salvadori, E., Roboti, P., Nyathi, Y., Muench, J.H., Roessler, M.M., et al. (2018). Structural complexity of the co-chaperone SGTA: a conserved C-terminal region is implicated in dimerization and substrate quality control. BMC Biology 16, 76.

Mast, F.D., Fagarasanu, A., Knoblach, B., and Rachubinski, R.A. (2010). Peroxisome Biogenesis: Something Old, Something New, Something Borrowed. Physiology 25, 347356.

Mazzarello, P., Calligaro, A., Vannini, V., and Muscatello, U. (2003). The sarcoplasmic reticulum: its discovery and rediscovery. Nature Reviews Molecular Cell Biology 4, 69 74.

McGraw, C.F., Somlyo, A.V., and Blaustein, M.P. (1980). Localization of calcium in presynaptic nerve terminals. An ultrastructural and electron microprobe analysis. The Journal of Cell Biology 85, 228-241.

Meng, W., and Takeichi, M. (2009). Adherens Junction: Molecular Architecture and Regulation. Cold Spring Harb Perspect Biol 1, a002899.

Meunier, L., Usherwood, Y.-K., Chung, K.T., and Hendershot, L.M. (2002). A Subset of Chaperones and Folding Enzymes Form Multiprotein Complexes in Endoplasmic Reticulum to Bind Nascent Proteins. MBoC 13, 4456-4469.

Michele, D.E., Barresi, R., Kanagawa, M., Saito, F., Cohn, R.D., Satz, J.S., Dollar, J., Nishino, I., Kelley, R.I., Somer, H., et al. (2002). Post-translational disruption of dystroglycan-ligand interactions in congenital muscular dystrophies. Nature 418, 417. 
Minami, R., Hayakawa, A., Kagawa, H., Yanagi, Y., Yokosawa, H., and Kawahara, H. (2010). BAG-6 is essential for selective elimination of defective proteasomal substrates. J. Cell Biol. 190, 637-650.

Mittl, P.R.E., and Schneider-Brachert, W. (2007). Sel1-like repeat proteins in signal transduction. Cellular Signalling 19, 20-31.

Molinari, M., and Helenius, A. (1999). Glycoproteins form mixed disulphides with oxidoreductases during folding in living cells. Nature 402, 90-93.

Molinari, M., and Helenius, A. (2000). Chaperone Selection During Glycoprotein Translocation into the Endoplasmic Reticulum. Science 288, 331-333.

Mostov, K.E., DeFoor, P., Fleischer, S., and Blobel, G. (1981). Co-translational membrane integration of calcium pump protein without signal sequence cleavage. Nature $292,87$.

Mouyna, I., Kniemeyer, O., Jank, T., Loussert, C., Mellado, E., Aimanianda, V., Beauvais, A., Wartenberg, D., Sarfati, J., Bayry, J., et al. (2010). Members of protein Omannosyltransferase family in Aspergillus fumigatus differentially affect growth, morphogenesis and viability. Molecular Microbiology 76, 1205-1221.

Mueller, B., Klemm, E.J., Spooner, E., Claessen, J.H., and Ploegh, H.L. (2008). SEL1L nucleates a protein complex required for dislocation of misfolded glycoproteins. PNAS $105,12325-12330$.

Muto, T., Obita, T., Abe, Y., Shodai, T., Endo, T., and Kohda, D. (2001). NMR identification of the Tom 20 binding segment in mitochondrial presequences11Edited by M. F. Sumers. Journal of Molecular Biology 306, 137-143.

Nandadasa, S., Tao, Q., Menon, N.R., Heasman, J., and Wylie, C. (2009). N- and Ecadherins in Xenopus are specifically required in the neural and non-neural ectoderm, respectively, for F-actin assembly and morphogenetic movements. Development 136, $1327-1338$.

Ni, M., and Lee, A.S. (2007). ER chaperones in mammalian development and human diseases. FEBS Lett 581, 3641-3651.

Nielsen, H. (2017). Predicting Secretory Proteins with SignalP. In Protein Function Prediction: Methods and Protocols, D. Kihara, ed. (New York, NY: Springer New York), pp. 59-73.

Nielsen, H., Engelbrecht, J., Brunak, S., and von Heijne, G. (1997). Identification of prokaryotic and eukaryotic signal peptides and prediction of their cleavage sites. Protein Eng. 10, 1-6. 
Nieuwkoop, P.D., Faber, J. (1994). Normal table of Xenopus laevis (Daudin): a systematical and chronological survey of the development from the fertilized egg till the end of metamorphosis (New York: Garland Pub).

O'Connor, S.E., and Imperiali, B. (1996). Modulation of protein structure and function by asparagine-linked glycosylation. Chemistry \& Biology 3, 803-812.

Odunuga, O.O., Longshaw, V.M., and Blatch, G.L. (2004). Hop: more than an Hsp70/Hsp90 adaptor protein. BioEssays 26, 1058-1068.

Palade, G.E., and Porter, K.R. (1954). Studies on the Endoplasmic Reticulum. J Exp Med $100,641-656$.

Palade, G.E., and Siekevitz, P. (1956). Liver Microsomes: An Integrated Morphological and Biochemical Study. The Journal of Cell Biology 2, 171-200.

Park, E., and Rapoport, T.A. (2012). Mechanisms of Sec61/SecY-Mediated Protein Translocation Across Membranes. Annual Review of Biophysics 41, 21-40.

Pearse, B.R., Gabriel, L., Wang, N., and Hebert, D.N. (2008). A cell-based reglucosylation assay demonstrates the role of GT1 in the quality control of a maturing glycoprotein. The Journal of Cell Biology 181, 309-320.

Petkova, M.I., Pujol-Carrion, N., and de la Torre-Ruiz, M.A. (2012). Mtl1 Omannosylation mediated by both Pmt1 and Pmt2 is important for cell survival under oxidative conditions and TOR blockade. Fungal Genetics and Biology 49, 903-914.

Petrova, K., Oyadomari, S., Hendershot, L.M., and Ron, D. (2008). Regulated association of misfolded endoplasmic reticulum lumenal proteins with P58/DNAJc3. The EMBO Journal 27, 2862-2872.

Pfaffl, M.W. (2001). A new mathematical model for relative quantification in real-time RT-PCR. Nucleic Acids Res 29, e45.

Pichler, H., Gaigg, B., Hrastnik, C., Achleitner, G., Kohlwein, S.D., Zellnig, G., Perktold, A., and Daum, G. (2001). A subfraction of the yeast endoplasmic reticulum associates with the plasma membrane and has a high capacity to synthesize lipids. European Journal of Biochemistry 268, 2351-2361.

Pobre, K.F.R., Poet, G.J., and Hendershot, L.M. (2018). The endoplasmic reticulum (ER) chaperone $\mathrm{BiP}$ is a master regulator of ER functions: Getting by with a little help from ERdj friends. J. Biol. Chem. 2098-2108.

Ponting, C.P. (2000). Novel repeats in ryanodine and IP 3 receptors and protein O mannosyltransferases. Trends in Biochemical Sciences 25, 47-50. 
Pravata, V.M., Muha, V., Gundogdu, M., Ferenbach, A.T., Kakade, P.S., Vandadi, V., Wilmes, A.C., Borodkin, V.S., Joss, S., Stavridis, M.P., et al. (2019). Catalytic deficiency of O-GlcNAc transferase leads to X-linked intellectual disability. PNAS 116, 1496114970.

Preissler, S., Rato, C., Chen, R., Antrobus, R., Ding, S., Fearnley, I.M., and Ron, D. (2015). AMPylation matches BiP activity to client protein load in the endoplasmic reticulum. ELife 4, e12621.

Preissler, S., Rato, C., Perera, L.A., Saudek, V., and Ron, D. (2016a). FICD acts bifunctionally to AMPylate and de-AMPylate the endoplasmic reticulum chaperone BiP. Nature Structural \&Amp; Molecular Biology 24, 23.

Preissler, S., Rohland, L., Yan, Y., Chen, R., Read, R.J., and Ron, D. (2016b). AMPylation targets the rate-limiting step of BiP's ATPase cycle for its functional inactivation. ELife 6.

Preissler, S., Rato, C., Perera, L.A., Saudek, V., and Ron, D. (2017). FICD acts bifunctionally to AMPylate and de-AMPylate the endoplasmic reticulum chaperone BiP. Nature Structural \& Molecular Biology 24, 23-29.

Price, J.L., Culyba, E.K., Chen, W., Murray, A.N., Hanson, S.R., Wong, C.-H., Powers, E.T., and Kelly, J.W. (2012). N-glycosylation of enhanced aromatic sequons to increase glycoprotein stability. Peptide Science 98, 195-211.

Proszynski, T.J., Simons, K., and Bagnat, M. (2004). O-Glycosylation as a Sorting Determinant for Cell Surface Delivery in Yeast. MBoC 15, 1533-1543.

Quellhorst, G.J., O’Rear, J.L., Cacan, R., Verbert, A., and Krag, S.S. (1999).

Nonglucosylated oligosaccharides are transferred to protein in MI8-5 Chinese hamster ovary cells. Glycobiology 9, 65-72.

Racapé, M., Huyen, J.-P.D.V., Danger, R., Giral, M., Bleicher, F., Foucher, Y., Pallier, A., Pilet, P., Tafelmeyer, P., Ashton-Chess, J., et al. (2011). The Involvement of SMILE/TMTC3 in Endoplasmic Reticulum Stress Response. PLOS ONE 6, e19321.

Rambaruth, N.D.S., and Dwek, M.V. (2011). Cell surface glycan-lectin interactions in tumor metastasis. Acta Histochemica 113, 591-600.

Rizzuto, R., Pinton, P., Carrington, W., Fay, F.S., Fogarty, K.E., Lifshitz, L.M., Tuft, R.A., and Pozzan, T. (1998). Close Contacts with the Endoplasmic Reticulum as Determinants of Mitochondrial Ca2+ Responses. Science 280, 1763-1766.

Roberts, J.D., Thapaliya, A., Martínez-Lumbreras, S., Krysztofinska, E.M., and Isaacson, R.L. (2015). Structural and Functional Insights into Small, Glutamine-Rich,

Tetratricopeptide Repeat Protein Alpha. Front. Mol. Biosci. 2. 
Roy, B., and Lee, A.S. (1999). The mammalian endoplasmic reticulum stress response element consists of an evolutionarily conserved tripartite structure and interacts with a novel stress-inducible complex. Nucleic Acids Res 27, 1437-1443.

Runge, C.L., Indap, A., Zhou, Y., Kent, J.W., King, E., Erbe, C.B., Cole, R., Littrell, J., Merath, K., James, R., et al. (2016). Association of TMTC2 With Human Nonsyndromic Sensorineural Hearing Loss. JAMA Otolaryngol Head Neck Surg 142, 866-872.

Rutkowski, D.T., Kang, S.-W., Goodman, A.G., Garrison, J.L., Taunton, J., Katze, M.G., Kaufman, R.J., and Hegde, R.S. (2007). The Role of p58IPK in Protecting the Stressed Endoplasmic Reticulum. Mol Biol Cell 18, 3681-3691.

Sanders, S.L., Gentzsch, M., Tanner, W., and Herskowitz, I. (1999). O-Glycosylation of Ax12/Bud10p by Pmt4p Is Required for Its Stability, Localization, and Function in Daughter Cells. The Journal of Cell Biology 145, 1177-1188.

Sanyal, A., Chen, A.J., Nakayasu, E.S., Lazar, C.S., Zbornik, E.A., Worby, C.A., Koller, A., and Mattoo, S. (2015). A Novel Link Between Fic (Filamentation induced by cAMP)mediated Adenylylation/AMPylation and the Unfolded Protein Response. J. Biol. Chem. 8482-8499.

Satoh, T., Ross, C.A., Villa, A., Supattapone, S., Pozzan, T., Snyder, S.H., and Meldolesi, J. (1990). The inositol 1,4,5,-trisphosphate receptor in cerebellar Purkinje cells: quantitative immunogold labeling reveals concentration in an ER subcompartment. The Journal of Cell Biology 111, 615-624.

Sawyer, N., Chen, J., and Regan, L. (2013). All Repeats are Not Equal: A Module-Based Approach to Guide Repeat Protein Design. J Mol Biol 425, 1826-1838.

Scheufler, C., Brinker, A., Bourenkov, G., Pegoraro, S., Moroder, L., Bartunik, H., Hartl, F.U., and Moarefi, I. (2000). Structure of TPR Domain-Peptide Complexes: Critical Elements in the Assembly of the Hsp70-Hsp90 Multichaperone Machine. Cell 101, 199210.

Schjoldager, K.T.-B.G., and Clausen, H. (2012). Site-specific protein O-glycosylation modulates proprotein processing - Deciphering specific functions of the large polypeptide GalNAc-transferase gene family. Biochimica et Biophysica Acta (BBA) General Subjects 1820, 2079-2094.

Schnell, D.J., and Hebert, D.N. (2003). Protein Translocons: Multifunctional Mediators of Protein Translocation across Membranes. Cell 112, 491-505.

Session, A.M., Uno, Y., Kwon, T., Chapman, J.A., Toyoda, A., Takahashi, S., Fukui, A., Hikosaka, A., Suzuki, A., Kondo, M., et al. (2016). Genome evolution in the allotetraploid frog Xenopus laevis. Nature 538, 336-343. 
Shafi, R., Iyer, S.P.N., Ellies, L.G., O’Donnell, N., Marek, K.W., Chui, D., Hart, G.W., and Marth, J.D. (2000). The O-GlcNAc transferase gene resides on the X chromosome and is essential for embryonic stem cell viability and mouse ontogeny. PNAS 97, 57355739 .

Shao, S., Rodrigo-Brenni, M.C., Kivlen, M.H., and Hegde, R.S. (2017). Mechanistic basis for a molecular triage reaction. Science 355, 298-302.

Shibata, Y., Shemesh, T., Prinz, W.A., Palazzo, A.F., Kozlov, M.M., and Rapoport, T.A. (2010). Mechanisms determining the morphology of the peripheral ER. Cell 143, 774788.

Shibatani, T., David, L.L., McCormack, A.L., Frueh, K., and Skach, W.R. (2005). Proteomic Analysis of Mammalian Oligosaccharyltransferase Reveals Multiple Subcomplexes that Contain Sec61, TRAP, and Two Potential New Subunits. Biochemistry 44, 5982-5992.

Shinjo, S., Mizotani, Y., Tashiro, E., and Imoto, M. (2013). Comparative Analysis of the Expression Patterns of UPR-Target Genes Caused by UPR-Inducing Compounds. Bioscience, Biotechnology, and Biochemistry 77, 729-735.

Siegenthaler, J.A., and Pleasure, S.J. (2011). We have got you "covered": how the meninges control brain development. Curr Opin Genet Dev 21, 249-255.

Sievers, F., Wilm, A., Dineen, D., Gibson, T.J., Karplus, K., Li, W., Lopez, R., McWilliam, H., Remmert, M., Soding, J., et al. (2014). Fast, scalable generation of highquality protein multiple sequence alignments using Clustal Omega. Molecular Systems Biology 7, 539-539.

Simmen, T., Lynes, E.M., Gesson, K., and Thomas, G. (2010). Oxidative protein folding in the endoplasmic reticulum: Tight links to the mitochondria-associated membrane (MAM). Biochimica et Biophysica Acta (BBA) - Biomembranes 1798, 1465-1473.

Simon, A.C., Simpson, P.J., Goldstone, R.M., Krysztofinska, E.M., Murray, J.W., High, S., and Isaacson, R.L. (2013). Structure of the Sgt2/Get5 complex provides insights into GET-mediated targeting of tail-anchored membrane proteins. PNAS 110, 1327-1332.

Solá, R.J., and Griebenow, K. (2009). Effects of glycosylation on the stability of protein pharmaceuticals. Journal of Pharmaceutical Sciences 98, 1223-1245.

Stein, O., and Stein, Y. (1967). Lipid Synthesis, Intracellular Transport, Storage, and Secretion. J Cell Biol 33, 319-339.

Strahl-Bolsinger, S., Immervoll, T., Deutzmann, R., and Tanner, W. (1993). PMT1, the gene for a key enzyme of protein O-glycosylation in Saccharomyces cerevisiae. PNAS 90, 8164-8168. 
Suisse, A., and Treisman, J.E. (2019). Reduced SERCA Function Preferentially Affects Wnt Signaling by Retaining E-Cadherin in the Endoplasmic Reticulum. Cell Reports 26, 322-329.e3.

Sun, S., Shi, G., Han, X., Francisco, A.B., Ji, Y., Mendonça, N., Liu, X., Locasale, J.W., Simpson, K.W., Duhamel, G.E., et al. (2014). SellL is indispensable for mammalian endoplasmic reticulum-associated degradation, endoplasmic reticulum homeostasis, and survival. PNAS 111, E582-E591.

Sunryd, Johan, C. (2014). The Identification and Characterization of a Group of ER TPRcontaining Adaptor Proteins. University of Massachusetts, Amherst

Sunryd, J.C., Cheon, B., Graham, J.B., Giorda, K.M., Fissore, R.A., and Hebert, D.N. (2014). TMTC1 and TMTC2 are novel endoplasmic reticulum TPR-containing adapter proteins involved in calcium homeostasis. J. Biol. Chem. 16085-16099.

Svärd, M., Biterova, E.I., Bourhis, J.-M., and Guy, J.E. (2011). The Crystal Structure of the Human Co-Chaperone P58IPK. PLOS ONE 6, e22337.

Takei, K., Stukenbrok, H., Metcalf, A., Mignery, G.A., Sudhof, T.C., Volpe, P., and Camilli, P.D. (1992). Ca2+ stores in Purkinje neurons: endoplasmic reticulum subcompartments demonstrated by the heterogeneous distribution of the InsP3 receptor, $\mathrm{Ca}(2+)$-ATPase, and calsequestrin. J. Neurosci. 12, 489-505.

Tannous, A., Pisoni, G.B., Hebert, D.N., and Molinari, M. (2015). N-linked sugarregulated protein folding and quality control in the ER. Seminars in Cell \& Developmental Biology 41, 79-89.

Tao, J., Petrova, K., Ron, D., and Sha, B. (2010). Crystal Structure of P58(IPK) TPR Fragment Reveals the Mechanism for its Molecular Chaperone Activity in UPR. Journal of Molecular Biology 397, 1307-1315.

Thapaliya, A., Nyathi, Y., Martínez-Lumbreras, S., Krysztofinska, E.M., Evans, N.J., Terry, I.L., High, S., and Isaacson, R.L. (2016). SGTA interacts with the proteasomal ubiquitin receptor Rpn13 via a carboxylate clamp mechanism. Scientific Reports 6, 36622.

Tripathi, A., Mandon, E.C., Gilmore, R., and Rapoport, T.A. (2017). Two alternative binding mechanisms connect the protein translocation Sec71/Sec72 complex with heat shock proteins. J. Biol. Chem. jbc.M116.761122.

Truebestein, L., and Leonard, T.A. (2016). Coiled-coils: The long and short of it. Bioessays 38, 903-916.

Truttmann, M.C., and Ploegh, H.L. (2017). rAMPing Up Stress Signaling: Protein AMPylation in Metazoans. Trends in Cell Biology 27, 608-620. 
Uhlén, M., Fagerberg, L., Hallström, B.M., Lindskog, C., Oksvold, P., Mardinoglu, A., Sivertsson, Å., Kampf, C., Sjöstedt, E., Asplund, A., et al. (2015). Tissue-based map of the human proteome. Science 347, 1260419.

Varki, A., and Sharon, N. (2009). Historical Background and Overview. In Essentials of Glycobiology, A. Varki, R.D. Cummings, J.D. Esko, H.H. Freeze, P. Stanley, C.R. Bertozzi, G.W. Hart, and M.E. Etzler, eds. (Cold Spring Harbor (NY): Cold Spring Harbor Laboratory Press), p.

Veratti, E. (1961). Investigations on the Fine Structure of Striated Muscle Fiber. The Journal of Cell Biology 10, 1-59.

Vester-Christensen, M.B., Halim, A., Joshi, H.J., Steentoft, C., Bennett, E.P., Levery, S.B., Vakhrushev, S.Y., and Clausen, H. (2013). Mining the O-mannose glycoproteome reveals cadherins as major O-mannosylated glycoproteins. Proceedings of the National Academy of Sciences 110, 21018-21023.

Voeltz, G.K., Rolls, M.M., and Rapoport, T.A. (2002). Structural organization of the endoplasmic reticulum. EMBO Rep 3, 944-950.

Walter, P., and Ron, D. (2011). The Unfolded Protein Response: From Stress Pathway to Homeostatic Regulation. Science 334, 1081-1086.

Wang, Y., Ju, T., Ding, X., Xia, B., Wang, W., Xia, L., He, M., and Cummings, R.D. (2010). Cosmc is an essential chaperone for correct protein O-glycosylation. PNAS 107, 9228-9233.

Willer, T., Prados, B., Falcón-Pérez, J.M., Renner-Müller, I., Przemeck, G.K.H., Lommel, M., Coloma, A., Valero, M.C., Angelis, M.H. de, Tanner, W., et al. (2004). Targeted disruption of the Walker-Warburg syndrome gene Pomt1 in mouse results in embryonic lethality. PNAS 101, 14126-14131.

Willer, T., Brandl, M., Sipiczki, M., and Strahl, S. (2005). Protein O-mannosylation is crucial for cell wall integrity, septation and viability in fission yeast. Molecular Microbiology 57, 156-170.

Wing, D.R., Rademacher, T.W., Schmitz, B., Schachner, M., and Dwek, R.A. (1992). Comparative glycosylation in neural adhesion molecules. Biochemical Society Transactions 20, 386-390.

Winklbauer, R. (2012). Cadherin Function During Xenopus Gastrulation. In Adherens Junctions: From Molecular Mechanisms to Tissue Development and Disease, T. Harris, ed. (Dordrecht: Springer Netherlands), pp. 301-320.

Worby, C.A., Mattoo, S., Kruger, R.P., Corbeil, L.B., Koller, A., Mendez, J.C., Zekarias, B., Lazar, C., and Dixon, J.E. (2009). The Fic Domain: Regulation of Cell Signaling by Adenylylation. Molecular Cell 34, 93-103. 
Wu, Y., and Sha, B. (2006). Crystal structure of yeast mitochondrial outer membrane translocon member Tom70p. Nat. Struct. Mol. Biol. 13, 589-593.

Wu, X., Cabanos, C., and Rapoport, T.A. (2018). Structure of the post-translational protein translocation machinery of the ER membrane. Nature 1.

$\mathrm{Xu}, \mathrm{C}$., and Ng, D.T.W. (2015). O-mannosylation: The other glycan player of ER quality control. Seminars in Cell \& Developmental Biology 41, 129-134.

Xu, C., Wang, S., Thibault, G., and Ng, D.T.W. (2013). Futile Protein Folding Cycles in the ER Are Terminated by the Unfolded Protein O-Mannosylation Pathway. Science 340, 978-981.

Yagi, T. (2008). Clustered protocadherin family. Development, Growth \& Differentiation 50, S131-S140.

Yan, W., Frank, C.L., Korth, M.J., Sopher, B.L., Novoa, I., Ron, D., and Katze, M.G. (2002). Control of PERK eIF2 $\alpha$ kinase activity by the endoplasmic reticulum stressinduced molecular chaperone P58IPK. PNAS 99, 15920-15925.

Yang, H.-Y., Tatebayashi, K., Yamamoto, K., and Saito, H. (2009). Glycosylation defects activate filamentous growth Kss 1 MAPK and inhibit osmoregulatory Hog1 MAPK. EMBO J 28, 1380-1391.

Yang, X., Zhang, F., and Kudlow, J.E. (2002). Recruitment of O-GlcNAc Transferase to Promoters by Corepressor mSin3A: Coupling Protein O-GlcNAcylation to Transcriptional Repression. Cell 110, 69-80.

Yi, F., Doudevski, I., and Regan, L. (2010). HOP is a monomer: Investigation of the oligomeric state of the co-chaperone HOP. Protein Sci 19, 19-25.

Yoshida, A., Kobayashi, K., Manya, H., Taniguchi, K., Kano, H., Mizuno, M., Inazu, T., Mitsuhashi, H., Takahashi, S., Takeuchi, M., et al. (2001). Muscular Dystrophy and Neuronal Migration Disorder Caused by Mutations in a Glycosyltransferase, POMGnT1. Developmental Cell 1, 717-724.

Young, J.C., Hoogenraad, N.J., and Hartl, F.U. (2003). Molecular Chaperones Hsp90 and Hsp70 Deliver Preproteins to the Mitochondrial Import Receptor Tom70. Cell 112, 4150 .

Zeytuni, N., and Zarivach, R. (2012). Structural and Functional Discussion of the TetraTrico-Peptide Repeat, a Protein Interaction Module. Structure 20, 397-405.

Zhang, H., and Hu, J. (2016). Shaping the Endoplasmic Reticulum into a Social Network. Trends in Cell Biology 26, 934-943. 
(2019). UniProt: a worldwide hub of protein knowledge. Nucleic Acids Res 47, D506D515. 